\title{
Comparisons of host susceptibility to isogenic virulent and hypovirulent [CHV1-infected] strains of Cryphonectria parasitica among Castanea hosts and plant tissue types
}

Cameron M. Stauder

Follow this and additional works at: https://researchrepository.wvu.edu/etd

\section{Recommended Citation}

Stauder, Cameron M., "Comparisons of host susceptibility to isogenic virulent and hypovirulent [CHV1-infected] strains of Cryphonectria parasitica among Castanea hosts and plant tissue types" (2016). Graduate Theses, Dissertations, and Problem Reports. 6718.

https://researchrepository.wvu.edu/etd/6718

This Thesis is protected by copyright and/or related rights. It has been brought to you by the The Research Repository @ WVU with permission from the rights-holder(s). You are free to use this Thesis in any way that is permitted by the copyright and related rights legislation that applies to your use. For other uses you must obtain permission from the rights-holder(s) directly, unless additional rights are indicated by a Creative Commons license in the record and/ or on the work itself. This Thesis has been accepted for inclusion in WVU Graduate Theses, Dissertations, and Problem Reports collection by an authorized administrator of The Research Repository @ WVU. For more information, please contact researchrepository@mail.wvu.edu. 
Comparisons of host susceptibility to isogenic virulent and hypovirulent [CHV1-infected] strains of Cryphonectria parasitica among Castanea hosts and plant tissue types

\title{
Cameron M. Stauder
}

Thesis submitted to the Davis College of Agriculture, Natural Resources and Design at West

Virginia University in partial fulfillment of the requirements for the degree of

\author{
Master of Science \\ in \\ Plant Pathology
}
William MacDonald, Ph.D., Chair Matthew Kasson, Ph.D., Chair
Daniel Panaccione, Ph.D. Fred Hebard, Ph.D.

Division of Plant and Soil Sciences

Morgantown, West Virginia

2016

Keywords: chestnut blight, host resistance, hypovirulence

Copyright 2016 Cameron Stauder 


\title{
ABSTRACT \\ Comparisons of host susceptibility to isogenic virulent and hypovirulent [CHV1-infected] strains of Cryphonectria parasitica among Castanea hosts and plant tissue types
}

\author{
Cameron M. Stauder
}

Chestnut blight is a devastating canker disease of numerous Castanea spp. resulting from the infection of wounds by Cryphonectria parasitica. The restoration of American chestnut has been pursued through the generation of blight resistant American $\mathrm{x}$ Chinese hybrids and employment of virulence attenuating hypoviruses of $C$. parasitica. The goals of this study were: 1 ) to conduct comparisons of host resistance among American ( $C$. dentata), European ( $C$. sativa), Chinese (C. mollisima), and three American $x$ Chinese hybrid generations (B2F2, B2F3, B3F2) produced by The American Chestnut Foundation (TACF) to isogenic virulent and hypovirulent (CHV1) strains of $C$. parasitica; 2 ) to validate the high-throughput use and reproducibility of the chestnut leaf susceptibility assay against the same fungal strains on representative leaves sampled across the various host backgrounds; and 3) to conduct comparisons among hypovirulent strains of $C$. parasitica using living stem inoculation, excisedleaf, and apple assays. The comparison of host resistance was conducted on populations of trees growing at the West Virginia University agronomy farm. Infections were initiated on livestems with a virulent strain designated 'Weekly' and an isogenic, hypovirulent 'Weekly-CHV1' strain (Euro7-CHV1). Subsequent canker measurements and fungal stromata counts were performed every two months for a year to assess host resistance. For virulent Weekly inoculations, Chinese chestnuts were often significantly more resistant, but few significant differences existed among the other hosts. Average linear growth measurements taken one year post-inoculation revealed B3F2 chestnuts to have the most linear growth of $20.02 \mathrm{~cm}$ and Chinese chestnuts to have the least linear growth of $13.92 \mathrm{~cm}$. Stromata density estimates were highly variable within each host background with the highest density on B3F2 at an average of 0.65 stromata $/ \mathrm{cm}^{2}$ and the least on Chinese chestnuts at an average of 0.25 stromata $/ \mathrm{cm}^{2}$. Additional measurements taken for virulent Weekly inoculations hinted at a higher level of resistance within the B2F2 hybrid generation while all other hosts aside from Chinese were often similar to American chestnut. Weekly-CHV1 cankers expanded during the first two months of the study, but no subsequent growth was observed on any host despite the recovery of these isolates nine months post-inoculation. An excised-leaf assay was conducted using leaves from a subset of trees included in the living stem assay. Weekly and Weekly-CHV1 were used to inoculate the midvein of leaves from all previously mentioned host backgrounds. There were no significant differences for the Weekly isolate inoculations, but the average lesion area for American chestnut $\left(78.5 \mathrm{~mm}^{2}\right)$ was largest and Chinese chestnut $\left(33.1 \mathrm{~mm}^{2}\right)$ was smallest among the tree species and breeding lines tested. For Weekly-CHV1 inoculations, Chinese chestnut $\left(42.7 \mathrm{~mm}^{2}\right)$ had significantly smaller lesion areas while all other hosts had average lesion areas similar to each other with the exception of B2F2 $\left(63.72 \mathrm{~mm}^{2}\right)$. Weekly $\left(58.1 \mathrm{~mm}^{2}\right)$ produced a significantly smaller average leaf-lesion area across all hosts than Weekly-CHV1 $\left(86.4 \mathrm{~mm}^{2}\right)$. Further, the incidence of infection was significantly lower for Weekly (39.5\%) than 
Weekly-CHV1 (79.1\%). The virulence of selected hypovirulent isolates also was examined through a living branch assay that employed a clonal clump of wild American chestnut sprouts, an excised-leaf assay using leaves from the same clonal clump, and an apple assay. WeeklyCHV1 once again produced significantly smaller cankers in the living branch assay than Weekly. Interestingly, Weekly-CHV1 produced larger lesions than Weekly in the leaf and apple assays while all other virulent strains produced larger lesions than their hypovirulent counterparts. Host resistance and pathogen virulence are the balancing point for the survival of an infected chestnut host. Here, a selection of American, European, TACF hybrid chestnuts were shown to be equally susceptible to stem infections of $C$. parasitica. The excised-leaf assay produced similar results with regards to host response, but hypovirulent Weekly-CHV1 unexpectedly produced larger lesions than virulent Weekly. This same observation was made for an apple assay and a second excised-leaf assay. These findings provide evidence for unique interactions between $C$. parasitica and hypoviruses not previously observed. 


\section{ACKNOWLEDGEMENTS}

Above all else, I must first thank God for every opportunity and blessing He has provided me throughout my life. While I often take all that I have been given for granted, I must recognize that my reality could be a much different one. As I reflect back on my academic career, I realize I likely would not have approached graduate school if Dr. Scott Schlarbaum had not advised me to continue my education. So, thank you for the push in the right direction and all of the guidance along the way. Much of my success would not have been possible without the wisdom, guidance, and patience of Dr. William MacDonald and Dr. Matthew Kasson. Thank you Dr. MacDonald for inviting me to study at WVU, welcoming Laura and me to Morgantown with open arms, and remaining a constant mentor during my time here. I also would like to thank Dr. Kasson for all of the adventures along the way. I knew I had chosen the right lab immediately after our first trip down to southern West Virginia to hunt Brachycybe millipedes. Thank you for always providing me with inspiration, knowledge, and friendship. I also would like to thank Dr. Daniel Panaccione and Dr. Fred Hebard for agreeing to serve as part of my graduate committee and for being patient and supportive with everything along the way. I must also extend a special thanks to Mark Double. Every lab needs a scientist like Mark, and I am very grateful for having been allowed to learn and laugh with him throughout my time here.

I would like to thank my beautiful wife Laura for being my constant companion throughout this journey. Every time I was down, you picked me up. You are forever my inspiration, and I love you. Next, I must thank my mother and father for always pushing me to pursue what I want in life rather than allowing me to settle for some other reality along the way. I could not have asked for better parents. Last but definitely not least, I would like to thank Kristen Wickert, Matt Berger, Amy Metheny, Angie Macias, and all of my other fellow graduate students and friends for making this adventure a fun one. I'll never forget our times running through forests, fields, and caves hunting for one thing or another. 


\section{TABLE OF CONTENTS}

ABSTRACT II-III

ACKNOWLEDGEMENTS

LIST OF TABLES. ...VII

LIST OF FIGURES. ..VIII-IX

LIST OF APPENDICES

IX

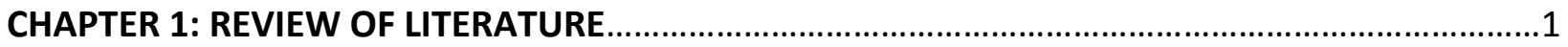

Brief history of the American chestnut and chestnut blight..............................................1

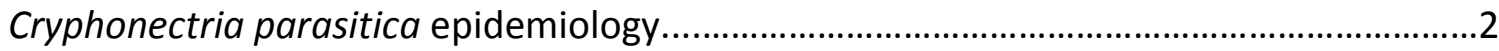

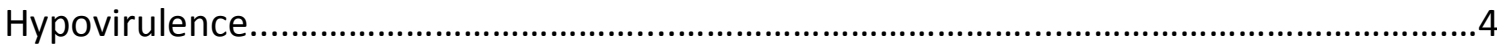

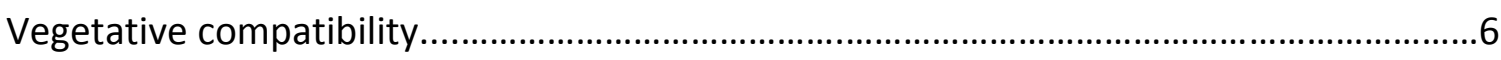

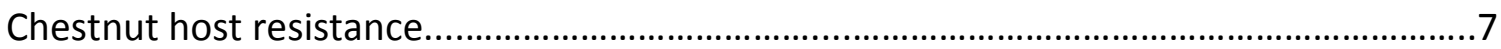

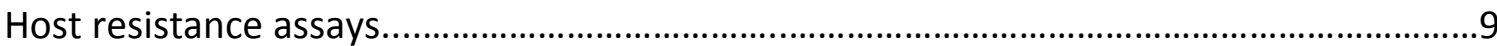

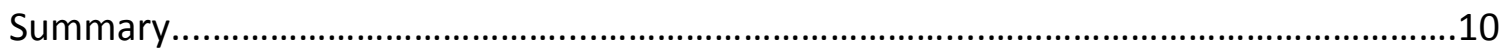

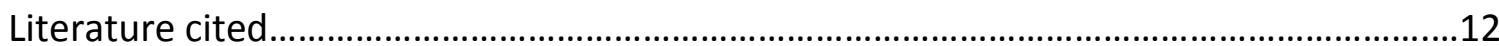

CHAPTER 2: COMPARISONS OF HOST SUSCEPTIBILITY TO DIRECT STEM INOCULATION OF ISOGENIC VIRULENT AND HYPOVIRULENT (CHV1) STRAINS OF CRYPHONECTRIA PARASITICA AMONG AMERICAN (CASTANEA DENTATA), CHINESE (C. MOLLISSIMA), EUROPEAN (C. SATIVA), AND AMERICAN X CHINESE HYBRID (B2F2, B2F3, B3F2) CHESTNUTS........................19

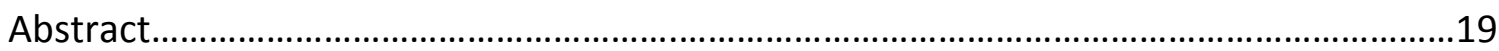

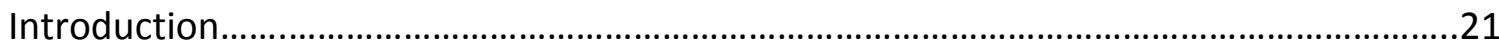

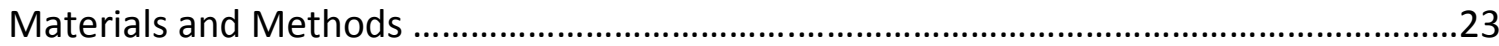

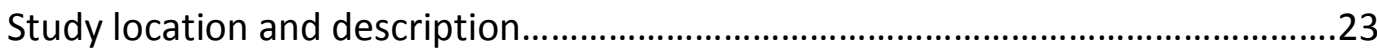

Experimental design and treatments...............................................................24

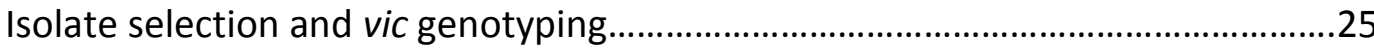

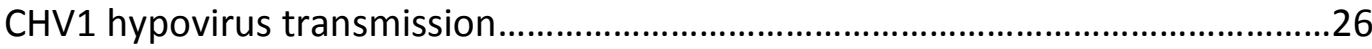

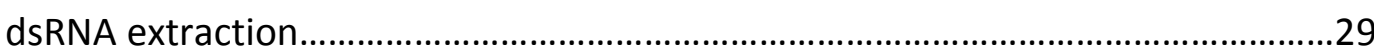

Inoculum preparation and inoculation procedure..................................................29

Canker linear growth and stromata estimates........................................................30

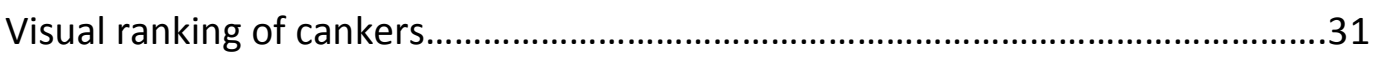

Tree diameter measurements and callus estimation...........................................32

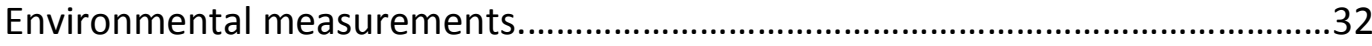

Cryphonectria parasitica reisolation and isolate confirmation...............................32

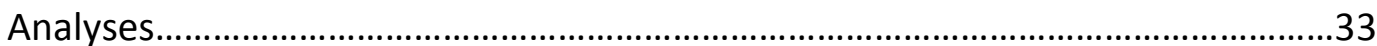

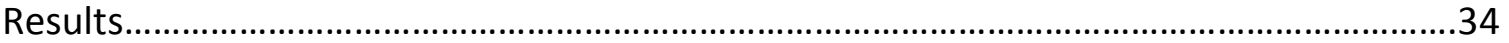

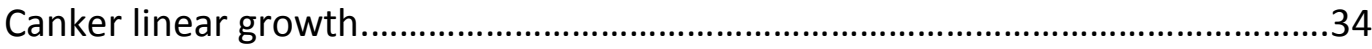




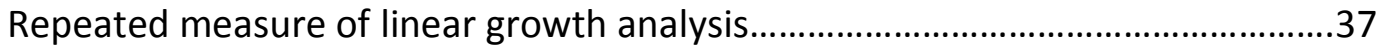

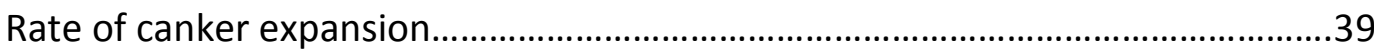

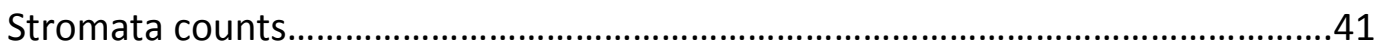

Effects of temperature and precipitation on sporulation and growth...................44

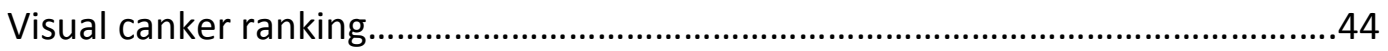

Stem diameter change and callus estimates........................................................45

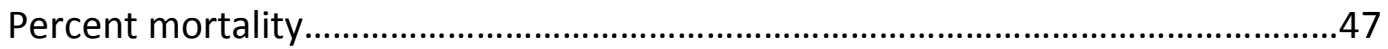

Reisolations from study cankers.........................................................................48

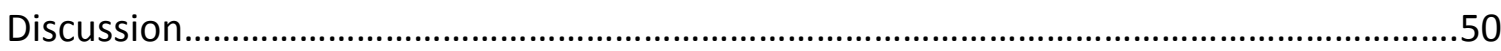

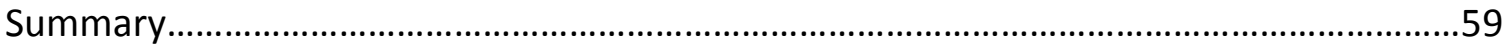

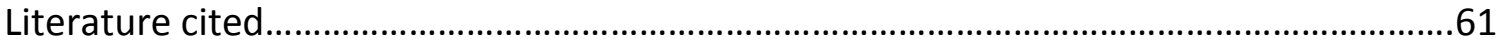

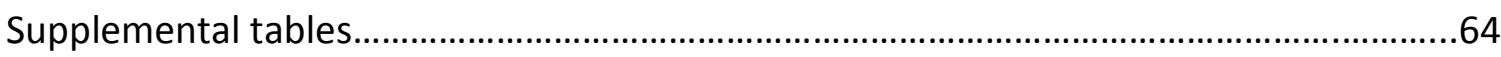

CHAPTER 3: EMPLOYING AN EXCISED-LEAF ASSAY TO ASSESS CHESTNUT HOST RESISTANCE

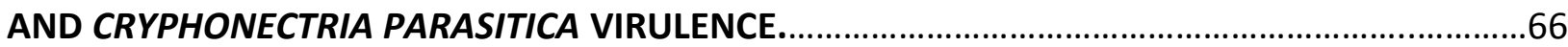

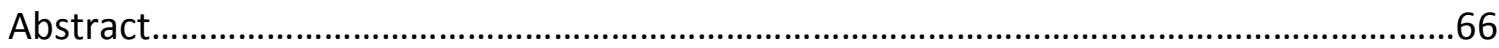

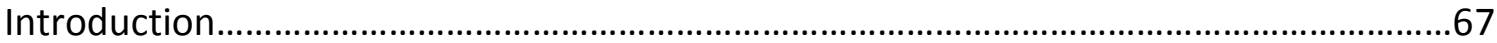

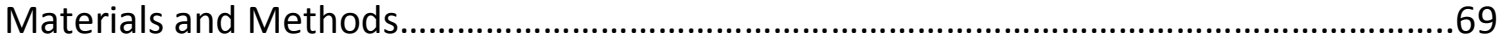

Experimental design and treatments............................................................69

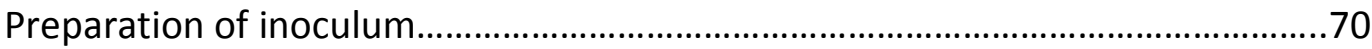

Collection, inoculation, and incubation procedure .................................................70

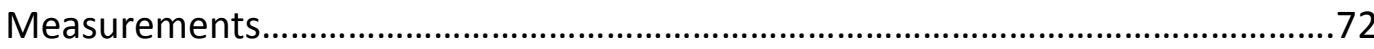

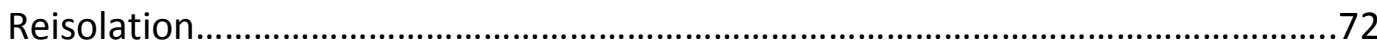

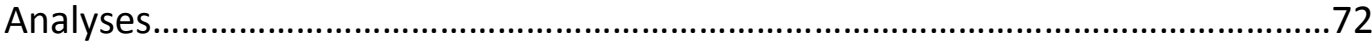

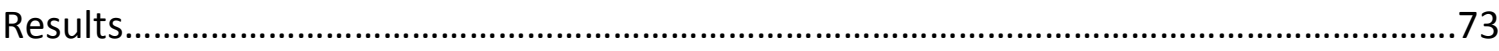

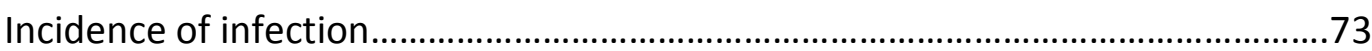

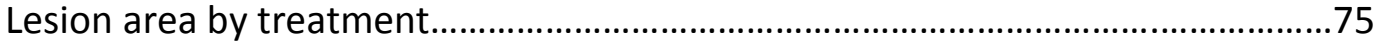

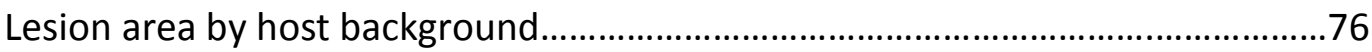

Comparison of normalized linear growth and leaf-lesion areas.............................78

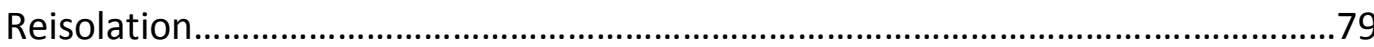

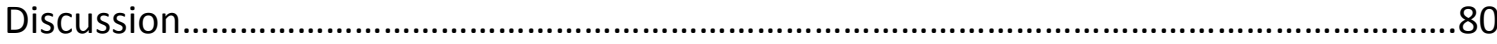

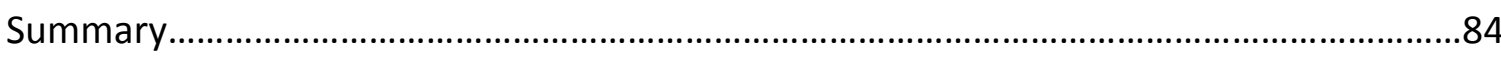

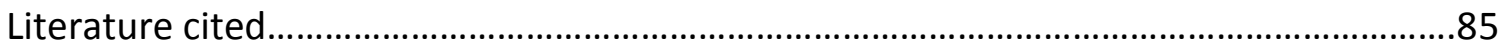

CHAPTER 4: THE INVASIVENESS OF VIRULENT AND HYPOVIRULENT (EURO7-CHV1) STRAINS OF CRYPHONECTRIA PARASITICA ON HOST AND NON-HOST SUBSTRATES..............................8

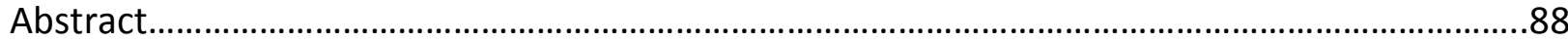

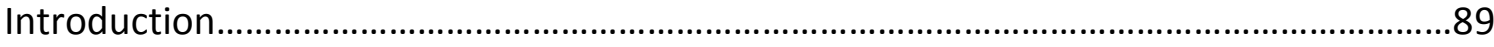




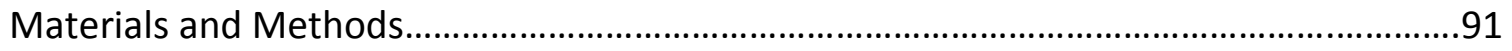

Live-stem assay isolate selection and hypovirus transmission................................91

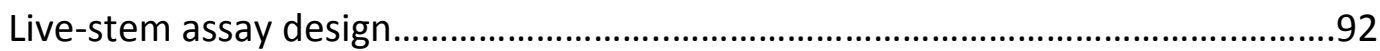

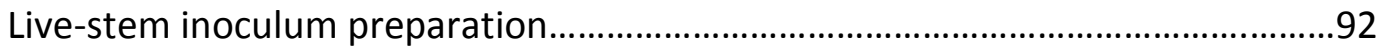

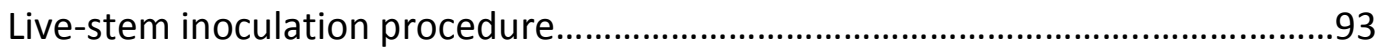

Excised-leaf and apple assay isolate selection and preparation............................93

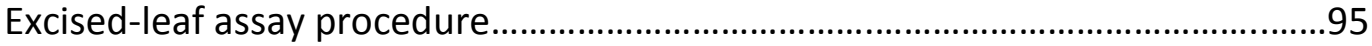

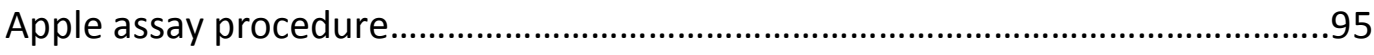

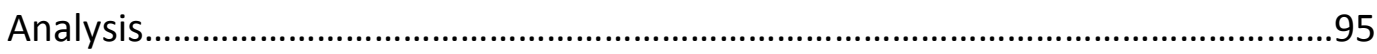

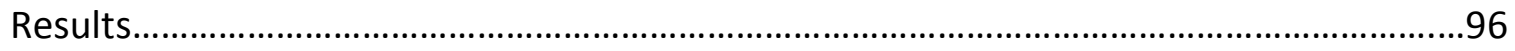

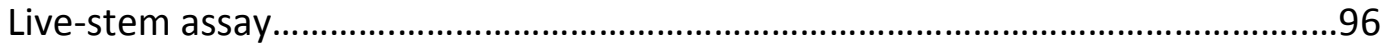

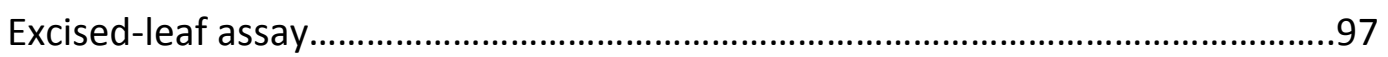

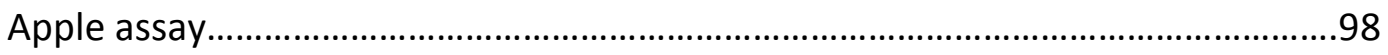

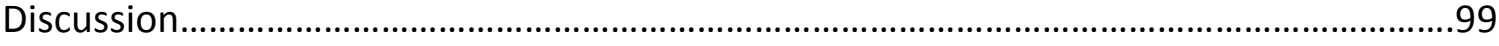

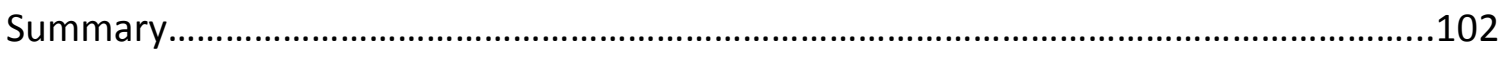

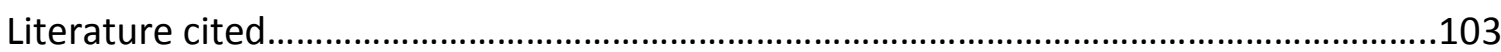

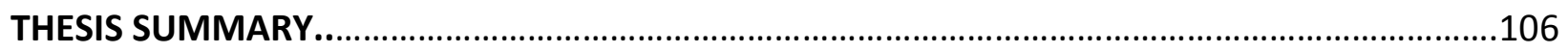

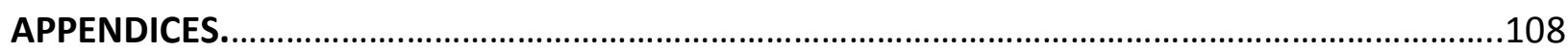

\section{LIST OF TABLES}

Chapter 2

Table 2.1: Table 2.1: Total trees by chestnut species or TACF hybrid generation.

Table 2.2: Vegetative incompatibility genotypes of selected virulent and hypovirulent

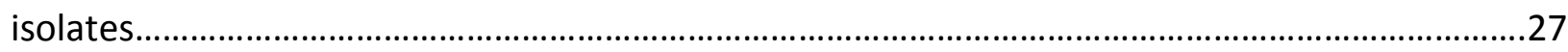

Table 2.3 Rank descriptions for visual assessment of cankers...................................................31

Table 2.4: Average precipitation and temperature values for each measurement period...........44

Table 2.5: Percent mortality at end of study by host background.................................................47

Table 2.6: Isolate genotype and dsRNA confirmation for reisolated $C$. parasitica isolates from selected cankers.

Chapter 4

Table 4.1: Designation and geographic origin of isolates included in the stem assay....... 


\section{LIST OF FIGURES}

\section{Chapter 2}

Figure 2.1: Aerial map of WVU Agronomy Farm with plot locations. .23

Figure 2.2: Excised stem assay results for selected virulent $C$. parasitica isolates

.26

Figure 2.3 (2a): Weekly x CHV1 isolate pairing with hypovirus transmission; (2b): Weekly-CHV1

(top) and Weekly virulent (bottom); (2c): Weekly paired with Weekly-CHV1.

Figure 2.4: Average linear growth $(\mathrm{I}+\mathrm{w} / 2 ; \mathrm{cm})$ for Weekly inoculations across the individual host backgrounds; error bars represent +/- $1 \mathrm{SE}$ 35

Figure 2.5: Average linear growth $(\mathrm{I}+\mathrm{w} / 2 ; \mathrm{cm})$ for Weekly-CHV1 inoculations across the individual host backgrounds; error bars represent +/- $1 \mathrm{SE}$.

Figure 2.6: Average linear growth $(\mathrm{I}+\mathrm{w} / 2 ; \mathrm{cm})$ for Weekly, Weekly-CHV1, and control inoculations; error bars represent +/- $1 \mathrm{SE}$

Figure 2.7: Average virulent Weekly linear growth $(\mathrm{cm})$ for each host background by measurement period; significance values located in the legend represent results from a repeated measures ANOVA.

Figure 2.8: Average virulent Weekly rate of canker expansion $(\mathrm{cm})$ for each host background across the six measurement periods of this study; error bars represent +/- $1 \mathrm{SE}$

Figure 2.9: Overall average rate of canker expansion for virulent Weekly for the six measurement periods of this study

Figure 2.10: Average stromata/ $\mathrm{cm}^{2}$ for individual host backgrounds; error bars represent $+/-1$

SE

Figure 2.11: Average stromata/ $\mathrm{cm}^{2}$ over time for individual host backgrounds. .43

Figure 2.12: Average stromata counts (stromata $/ \mathrm{cm}^{2}$ ) for all hosts by measurement period; error bars represent +/- $1 \mathrm{SE}$

Figure 2.13: Average virulent canker rank for each host background; error bars represent +/- 1 SE.

Figure 2.14: Diameter increase from start to end of study across all host backgrounds; error bars represent +/- $1 \mathrm{SE}$.

Figure 2.15: Callus growth estimate from diameter increase for virulent Weekly canker inoculation sites; error bars represent +/- 1 SE.

Figure 2.16: dsRNA extraction gel photo for selected reisolated hypovirulent isolates with the original Weekly CHV1 as a positive control.

Supplemental Figure 2.1: Comparison of average diameters of alive and dead hosts; error bars represent +/- 1 SE. .64

Supplemental Figure 2.2: Average linear growth 12-months post-inoculation by host background and lineage; error bars represent +/- $1 \mathrm{SE}$. .65

Supplemental Figure 2.3: Average total stromata counts across all host backgrounds; error bars represent +/- $1 \mathrm{SE}$. 


\section{Chapter 3}

Figure 3.1: Leaf assay procedure as described by Newhouse et. al (2014)....

Figure 3.2: Successful (A) and unsuccessful (B) Cryphonectria parasitica infections on chestnut leaves.

Figure 3.3: Average percent of successful infection for all host backgrounds for Weekly and Weekly-CHV1; Total percent infection by inoculum type was analyzed separately; error bars represent +/- $1 \mathrm{SE}$.

Figure 3.4: Leaf-lesion area $\left(\mathrm{mm}^{2}\right)$ averaged for all hosts for Weekly and Weekly-CHV1 inoculations; error bars represent +/- $1 \mathrm{SE}$ .75

Figure 3.5: Average leaf-lesion area $\left(\mathrm{mm}^{2}\right)$ for each host backgrounds for Weekly and WeeklyCHV1 inoculations; error bars represent +/- 1 SE.

Figure 3.6: Average leaf-lesion areas $\left(\mathrm{mm}^{2}\right)$ for virulent Weekly inoculations for each host background; error bars represent +/- $1 \mathrm{SE}$.

Figure 3.7: Average leaf-lesion area for Weekly-CHV1 inoculations across all host backgrounds; error bars represent +/- $1 \mathrm{SE}$. .78

Figure 3.8: Normalized values for Weekly leaf-lesion area and Weekly canker linear growth across all host backgrounds; error bars represent +/- $1 \mathrm{SE}$

\section{Chapter 4}

Figure 4.1: dsRNA extraction gel photo for hypovirulent isolates with virulent Weekly as a negative control

Figure 4.2: Linear growth at one month post-inoculation for virulent Weekly and six hypovirulent isolates included in the live-stem inoculations at Savage River State Forest; error bars represent +/- $1 \mathrm{SE}$.

Figure 4.3: Linear growth at twelve weeks post-inoculation for virulent Weekly and six hypovirulent isolates included in the live-stem inoculations at Savage River State Forest; error bars represent +/- $1 \mathrm{SE}$

Figure 4.4: Lesion areas for isolates included in the excised-leaf assay; error bars represent +/- 1 SE.

Figure 4.5: Average linear growth for all isolates included in the apple assay; error bars represent +/- $1 \mathrm{SE}$

\section{List of Appendices}




\section{CHAPTER 1: REVIEW OF LITERATURE}

\section{Brief history of the American chestnut and chestnut blight}

Chestnut blight is a devastating canker disease of Castanea spp. caused by Cryphonectria parasitica (Murr.) Barr, an introduced Ascomycotan fungus. Several other members of Fagaceae, such as scarlet oak (Quercus coccinea), also serve as hosts to $C$. parasitica although disease rarely results in mortality (Torsello et al., 1994). This disease was first observed by H.W. Merkel in 1904 in the New York City Zoological Park (Merkel, 1905). The pathogen decimated American chestnut (Castanea dentata (Marsh.) Borkh.) populations across the entire 3.6 million hectare range in eastern North America within 50 years at a dispersal rate of ca. 31 km/year (Anagnostakis, 1987; Evans and Finkral, 2010). Paradoxically, chestnut blight has led to the proliferation of American chestnuts in the understory in the form of vegetative sprouts, which are equally susceptible to blight, resulting in a repeated cycle of sprouting and death as infections reoccur.

The source of the introduction of $C$. parasitica into North America likely was an imported shipment of infected Japanese chestnut (C. crenata) seedlings (Anagnostakis, 1987; Dutech et al., 2012). Asiatic chestnut trees (C. mollissima and C. crenata) are hosts of $C$. parasitica but have a high level of resistance presumably due to their co-evolution with the pathogen in Asia, the presumed origin for C. parasitica. This observation, along with Approximate Bayesian Computation analyses, helped to reconstruct the origins and routes of $C$. parasitica colonization and thus strengthen evidence for the Japanese origin of the pathogen (Dutech et al., 2012). Although Asiatic chestnuts generally possess a high level of resistance, they lack many of the desirable qualities of American chestnut, such as its dominance in the 
forest canopy along with its straight, unbranched growth. Efforts are underway to breed resistance to $C$. parasitica into American chestnut by crossing $C$. dentata with $C$. mollisima and C. crenata (Hebard, 2005). More recent efforts by Powell and others have included the integration of resistance-enhancing genes from wheat into the American chestnut to generate a minimally modified, resistant American chestnut (Newhouse, 2014).

Approximately 45 years after the discovery of the disease in North America on American chestnut, $C$. parasitica was reported as causing the same disease on European chestnut trees (Castanea sativa Mill.) in Udine, Italy near Genoa (Pavari, 1949). Fortunately, the pathogen has had a less devastating effect on European populations likely due to a combination of a higher level of European chestnut's resistance to blight than American chestnut, a disjunct host range created by geographic barriers, and the limited diversity of vegetative compatibility (vc) types of $C$. parasitica in Europe, which allowed for the transmission of a debilitating hypovirus among compatible strains (Graves, 1950; Anagnostakis, 1986; Heiniger and Rigling, 1994).

\section{Cryphonectria parasitica epidemiology}

Cryphonectria parasitica typically infects through bark wounds (Anderson and Rankin, 1914). From the site of infection, the hyphae grow radially forming a mycelial fan resulting in cambial necrosis behind the advancing margin. The creation of necrotic tissue has been attributed to the excretion of oxalic acid by C. parasitica, which has a cell wall-degrading, toxic effect on host tissues (Havir and Anagnostakis, 1983).

The disease is similar to other canker diseases progressing from limited superficial lesions at onset followed by radial canker expansion. Prior to circumferential coalescence of the 
canker or cankers that result in stem girdling, blight cankers exhibit bark discoloration and often, swollen callus formation around the area of infection. As the mycelial fan of C. parasitica encircles the stem, the leaves of the stem distal to the canker rapidly wilt and become necrotic or 'blight' as the branch is girdled. Below the canker, epicormic sprouting often occurs as the host responds to the infection.

As a member of Ascomycota, the sexual (ascospores) and asexual (conidia) spores from spore-forming bodies known as perithecia and pycnidia, respectively, are formed in orange stromata that extrude from the bark. Given the sticky conidial ooze from their pycnidia, dispersal is likely most common through rain-water, insects, mammals, and birds (Anagnostakis, 1987). Ascospores are passively discharged from the perithecia and wind disseminated over longer distances (Heald et al., 1915).

Sexual reproduction in Ascomycota is unifactorial, controlled by a single mating-type locus with two alleles, MAT A and MAT a, that code for MAT-specific pheromones and receptors (Kues \& Casselton, 1992). Under these heterothallic conditions, an opposite mating type is required for a fungus to sexually reproduce and outcross. With C. parasitica, a mixed mating system can occur via mating-type heterokaryosis thus creating a self-fertile condition by which selfing can occur (McGuire et al., 2004). These heterokaryotic conditions are thought to form through recurrent biparental inbreeding or parasexual recombination (Milgroom et al., 2009). 


\section{$\underline{\text { Hypovirulence }}$}

The phenomenon of hypovirulence was first noted when 'spontaneously healing' cankers on European chestnut were observed in Italy by Biraghi (1953). Hypovirulence of C. parasitica is the result of infection of $C$. parasitica by one of four known mycoviruses of the family Hypoviridae. The viruses are characterized as double-stranded ribonucleic acid (dsRNA), cytoplasmically-restricted viruses, which lack a protein coat (Hillman, 2004). Hypovirulent strains of $C$. parasitica are generally characterized as having lighter pigment with attenuated growth and sporulation, which reduces canker development and reproductive capacity. These characteristics vary based on the infecting hypovirus and pathogen's genetics as well as the interactions between them (MacDonald and Fulbright, 1991).

Four hypovirus species have been described to date from C. parasitica in the Hypoviridae: Cryphonectria hypovirus 1 (CHV1) (Allemann et al., 1999; Heiniger and Rigling, 1994), CHV2 (Chung et al., 1994; Hillman, 1994), CHV3 (Smart et al., 1999), and CHV4 (Enebak, 1992; Linder-Basso, 2005). They are unique in their geographical occurrence, genomic structure, and effect on C. parasitica. Bauman (2015) described significant variations in the growth and asexual reproduction of strains infected with one of three hypoviruses: CHV3County Line, CHV1-Euro7 and CHV1-Ep713 with the conclusion that the host genome plays a significant role in hypovirulent conditions.

Hypovirulence is generally confirmed using such characteristics as attenuation of virulence, pigmentation, conidiation, oxalate accumulation, laccase production, and female fertility (Elliston, 1978; Anagnostakis, 1982; Havir, 1983; Rigling et al., 1989; Deng et al., 2007). Molecular confirmation of hypovirulent infection can be achieved via an extraction of the 
dsRNA virus (Morris and Dodds, 1979). CHV1 has been found in Europe and is considered partially responsible for the recovering European chestnut populations (Heiniger and Rigling, 1994; Milgroom and Cortesi, 2004). Like C. parasitica, the origin of these hypoviruses also is suspected to be from Asia where the CHV1 virus also has been discovered (Peever et al., 1998). CHV2 was found likely to be exclusive to New Jersey, and CHV3 has been predominately found in Michigan (Hillman et al., 1994; Smart et al., 1999). CHV4 has been found in isolates from throughout eastern North America including Kentucky, West Virginia, and New York but seems to have little to no effect on the pathogenicity or the biology of the blight fungus (Peever et al., 1997; Enebak, 1992).

Much variation in general morphology and pathogenic capacity is observed among individual hypovirulent strains of $C$. parasitica containing the same hypovirus species (Elliston, 1985; Peever et al., 2000). Interactions between the genetics of the hypovirus and its fungal host influence the extent to which a hypovirus can reduce overall pathogenicity of infected strains, including sporulation and growth (Nuss, 2005).

The transmission of hypoviruses can occur vertically and horizontally, where vertical transmission is defined as transmission between generations via spores while horizontal transmission is defined as transmission between individual thalli. Vertical transmission is restricted to the conidia given the lack of sexual reproduction in hypovirulent strains (Deng, 2007). The presence of the hypovirus in conidia is variable but is considered to be the major means of hypovirus dissemination (Peever et al. 2000; Prospero et al. 2006). Horizontal transmission is regulated by the occurrence of vegetative compatibility genes, which generally permit or deny hyphal anastomosis (Anagnostakis, 1982; Cortesi and Milgroom, 1998). 
Hypovirulence appeared promising as a biological control of chestnut blight and served as such in Europe as mentioned above. However, due to several complexities (see below), biological control has never been fully realized in the U.S. Instead, these hypovirulent strains are often used to directly treat virulent cankers as a means of transmitting the virus to often lethal causal strains.

Vegetative compatibility

The transmission of the hypovirus is dependent on the occurrence of hyphal anastomosis between two vegetatively compatible strains of $C$. parasitica (Van Alfen et al., 1975). Vegetative compatibility is based on a set of 6 unlinked, vegetative incompatibility (vic) loci (vic 1, 2, 3, 4, 6, \& 7), that are expressed as one of two alleles (1 or 2) (Cortesi and Milgroom, 1998). In theory, hyphae of strains that match exactly at these loci will readily fuse by anastomosis, and hypovirus transmission will occur through cytoplasmic mixing. Strains that differ at one locus are less likely to form this union, and this likelihood dramatically decreases with each additional difference (Liu and Milgroom, 1996). Furthermore, the degree of restriction of virus transmission is dependent on the specific locus. Each locus influences the occurrence of anastomosis differently and thus, hypovirus transmission (Cortesi et al., 2001). In addition to these six vic genes, there has been increasing evidence of additional vic genes and/or other genes in the pathogen's genetic background regulating hyphal anastomosis (Cortesi et al. 2001, Zamora et al. 2015, Short et. al 2015).

Based on the currently accepted hypothesis of six vic loci, $64\left(=2^{6}\right)$ vic genotypes could occur in nature. Generally, North America is considered to have much higher vic diversity than 
many areas of Europe. This is likely the result of higher vic-allelic diversity and higher rates of recombination in North America (Milgroom and Cortesi, 1999; Short et al. 2015). Also, areas recently invaded and colonized by C. parasitica are generally less diverse than longerestablished areas of infection, perhaps due to limited diversity in founder populations and the lack of subsequent introductions (Milgroom et al. 2008; Dutech et al., 2010). Further, the presence of a hypovirus in founder populations likely limits diversity of vic genotypes through the reduction of outcrossing via female sterility.

In 2015, Short et al. developed a multilocus PCR protocol that allows for vic genotyping. This advance allows for the rapid identification of vegetative compatibility based on each strains vic genotype. Given the vic genotypes found within a population, hypovirulent strains of matching vic genotypes can be employed to transmit hypovirus to present virulent infections as a means of biological control.

\section{Chestnut host resistance}

Graves (1950) made an early effort to characterize the levels of resistance among Castanea hosts to the blight pathogen. His findings supported a proposed ranking system, which placed Castanea spp. in the following order beginning with the most resistant: Chinese Class I; Japanese (C. crenata Sieb. \& Zucc.) - Class II; Seguin (C. sequini Dode.) - Classes II-IV; Allegany chinkapin (C. pumila Mill.) - Class III; Ozark chinkapin (C. ozarkensis Ashe.) - Classes III and IV; Henry chinkapin (C. henryi Rehd. \& Wils.) - Class IV; European (C. sativa) - Class IV; and America (C. dentata) - Class V. Subsequent studies based on inoculation of individual species 
with virulent strains of $C$. parasitica and measurements of resultant cankers generally validated Graves' ranking system (Anagnostakis, 1991; Berry, 1960; Clapper, 1952).

Within these studies, some limited, quantitative analyses were made. Berry (1960) found Chinese chestnut's average canker areas to be nearly $1 / 2$ that observed on European chestnuts and down to $1 / 3$ of the canker size on American chestnut. In a study by Anagnostakis (1991), American, Chinese, Japanese, Chinese chinkapin and various hybrids were compared based on canker width expansion rates ( $\mathrm{mm} /$ day). Her results show the following rates of expansion: American: $1 \mathrm{~mm} /$ day; Chinese: 0.2-0.6 mm/day; Chinese-American x American hybrid: 0.3-0.4 mm/day.

Upon the recognition of Asiatic chestnut's natural resistance to blight, early programs to develop resistant American chestnuts were initiated. These initial programs failed to successfully create a chestnut with the resistance of Asiatic chestnuts and the desired qualities of the American chestnut. This initiative was championed when Burnham et al. (1986) proposed that by backcrossing Chinese chestnut to the American chestnut resistance could be achieved. The American Chestnut Foundation would be formed, dedicated to testing the Burnham hypothesis (Hebard, 2005).

Backcross breeding is a method by which genes for blight resistance in Chinese chestnut can be integrated with the genes of the American chestnut to create an American $x$ Chinese hybrid. The process, as defined by Hebard (2005), begins by selecting individual Chinese and American chestnut trees that highly express the desired Chinese resistance and a desirable American phenotype. Individual representatives of each species are then crossed to create American $x$ Chinese progeny (F1, 50\% American). The progeny are then backcrossed with 
American chestnuts (B1, 75\% American). The B1 generation, as well as every subsequent generation following, are then evaluated and screened for resistance. The progeny are backcrossed with Americans twice more (B2, 87.5\% American; B3, 93.75\% American).

Once B3 hybrids are generated, a step known as intercrossing (i.e. B3 x B3) can be utilized to recover trees that are as homozygous for blight resistance, as they may inherit resistance genes from both parents. B3F2 trees that are homozygous for blight resistance should be true breeding for blight resistance thus most of their B3F3 progeny will be resistant to blight while the remaining B3F2s should segregate into various levels within the entire range of resistance between American and Chinese chestnut.

\section{$\underline{\text { Host resistance assays }}$}

Resistance assays are of particular importance to resistance breeding programs. Artificial branch inoculations of C. parasitica, as described by Jaynes and Elliston (1978), provide a method to assess resistance by comparing resultant cankers. While effective, this resistance screening method can encompass $>3$ years due to the need for the trees to grow to a suitable size and the time between inoculation and canker measurements (Griffin et al., 1983; Hebard, 2005).

Due to this long time period, methods of measuring host resistance in a shorter amount of time are desired. An excised bark/wood inoculation method has been used to produce measurable results in $\sim 4$ days (Lee et al., 1992), but this method also requires wounding the host (i.e. excising bark or branches) in such a way that might provide a means of entry for naturally occurring inoculum of $C$. parasitica. The inoculation of dormant excised stems can 
provide measurable results within 5 weeks (Elliston, 1978), but once again, a mode of entry is created on the tree. Despite the reasonably quick turnaround of these two methods, both require stems large enough to be sampled or utilized, and therefore, samples or stems are likely to be taken from clumps of vegetative sprouts or branches on larger trees thus making these methods less suitable for screening within resistance breeding programs which often maintain young saplings prior to screening.

For resistance breeding, the planting and maintenance of progeny prior to evaluation is time and resource intensive. Therefore, any method that might increase the efficiency of resistance evaluation is desirable. Newhouse et al. (2014) described an excised-leaf assay method by which measureable, host-specific representative results can be obtained within 4-7 days without compromising the host's integrity. Since the leaves are the tested tissues, resistance could be evaluated from seedling leaves within the first year. Therefore, trees that do not meet the desired level of resistance could be culled much earlier, and resources could be allocated elsewhere.

\section{Summary}

Even though Cryphonectria parasitica, hypovirulence, and the resistance of chestnut species have been extensively studied, the relative resistance of the TACF hybrid generations to virulent and hypovirulent strains, the practicability of the excised-leaf assay for identifying intermediate levels of resistance, and the variability of virulence among hypovirulent isolates need to be further studied. The objectives of this thesis were to: 1) conduct comparisons of host resistance across existing Castanea spp. and various American x Chinese crosses generated 
by The American Chestnut Foundation (TACF) using isogenic virulent and hypovirulent (CHV1) strains of $C$. parasitica; 2 ) validate the high-throughput use and reproducibility of the chestnut leaf susceptibility assay against the same fungal strains on representatives across the various host backgrounds; and 3) conduct comparisons among hypovirulent strains of $C$. parasitica using direct stem inoculation, excised-leaf, and apple assays. Addressing these topics will allow for further understanding of methodology to measure host resistance to chestnut blight. 


\section{LITERATURE CITED}

Allemann, C., P. Hoegger, U. Heiniger, and D. Rigling. 1999. Genetic variation of Cryphonectria hypoviruses (CHV1) in Europe, assessed using restriction fragment length polymorphisms (RFLP) markers. Molecular Ecololgy. 8(5):843-54.

Anagnostakis, S. L. 1982. Biological control of chestnut blight. Science. 215(4532):466-71.

Anagnostakis, S. L. 1982. Genetic analyses of Endothia parasitica: Linkage data for four single genes and three vegetative compatibility types. Genetics. 102: 25-28.

Anagnostakis, S. L., B. Hau, and J. Kranz. 1986. Diversity of vegetative compatibility groups of Cryphonectria parasitica in Connecticut and Europe. Plant Disease. 70:536-38.

Anagnostakis, S. L. 1987. Chestnut blight: the classical problem of an introduced pathogen. Mycologia. 79: 23-37.

Anagnostakis, S. L. 1991. Measuring resistance of chestnut trees to chestnut blight. Canadian Journal of Forest Research. 22:568-71.

Anderson, P.J. and W.H. Rankin. 1914. Endothia canker of chestnut. D.o.P. Pathology, ed. Ithaca, NY, USA: Cornell University, Agricultural Experiment Station of the College of Agriculture. 533-618.

Bauman, J. M. 2015. A comparison of the growth and asexual reproduction by Cryphonectria parastitica isolates infected with hypoviruses CHV3-County Line, CHV1Euro7, and CHV1-Ep713. American Journal of Plant Sciences. 6:73-83.

Berry, F. H. 1960. Relative resistance of some chestnut species and hybrids inoculated with the blight fungus. Plant Disease Reporter. 44(9):716-17.

Biraghi, A. 1953. Possible active resistance to Endothia parastitica in Castanea sativa. 
Reports of the International Union of Forest Research Organization. 643-45.

Burnham, C. R., Rutter, P. A. and French, D. W. (1986) Breeding Blight-Resistant

Chestnuts, in Plant Breeding Reviews, vol. 4 (ed J. Janick), John Wiley \& Sons, Inc., Hoboken, NJ, USA.

Clapper, R. B. 1952. Relative blight resistance of some species and hybrids. Journal of Forestry. 50:453-55.

Cortesi, P. and M. G. Milgroom. 1998. Genetics of vegetative incompatibility in Cryphonectria parasitica. Applied and Environmental Microbiology. 64(8):2988-94.

Cortesi, P., C. E. McCulloch, H. Song, H. Lin, and M. G. Milgroom. 2001. Genetic control of horizontal virus transmission in the chestnut blight fungus, Cryphonectria parasitica. Genetics. 159: 107-18.

Chung, P., P. J. Bedker, and B. I. Hillman. 1994. Diversity of Cryphonectria parasitica hypovirulence-associated double-stranded RNAs within a chestnut population in New Jersey. Phytopathology. 84(9): 984-990.

Deng, F., T. D. Allen, and D. L. Nuss. 2007. Ste12 transcription factor homologue CpST12 is down-regulated by hypovirus infection and required for virulence and female fertility of the chestnut Blight fungus Cryphonectria parasitica. Eukaryotic Cell. 6(2):235-44.

Dutech, C., O. Fabreguettes, X. Capdevielle, and C. Robin. 2010. Multiple introductions of divergent genetic lineages in an invasive fungal pathogen, Cryphonectria parasitica, in France. Heredity. 105:220-28.

Dutech, C, B. Barres, J. Bridier, C. Robin, M. G. Milgroom, and V. Ravigne. 2012. The chestnut blight fungus world tour: successive introduction events from diverse 
origins in an invasive plant fungal pathogen. Molecular Ecology. 21:3931-46.

Elliston, J. E. 1985. Characteristics of dsRNA-free and dsRNA-containing strains of Endothia parasitica in relation to hypovirulence. Phytopathology. 75:151-58

Elliston, J. E. 1978. Pathogenicity and sporulation in normal and diseases strains of Endothia parasitica in American chestnut. In Proceedings of the American Chestnut Symposium. W.L. MacDonald, F.C. Cech, J. Luchok, and C. Smith, eds. WV Univ. Books, Morgantown. 110-114.

Enebak, S.A. 1992. Characterization of dsRNA-containing strains of Cryphonectria parasitica recovered from the central Appalachian. PhD Thesis, West Virginia University, Morgantown.

Evans, A. M. and A. J. Finkral. 2010. A new look at spread rates of exotic diseases in North American forests. Forest Science. 56(5):453-59.

Graves, A. H. 1950. Relative blight resistance in species and hybrids of Castanea. Phytopathology. 40(12): 1125-31.

Griffin, G. J., F. V. Hebard, R. W. Wendt, and J. R. Elkins. 1983. Survival of American Chestnut trees: evaluation of blight resistance and virulence in Endothia parasitica. Phytopathology. 73(7):1084-92.

Havir, E. A. and S. L . Anagnostakis. 1983. Oxalate production by virulent but not by hypovirulent strains of Endothia parasitica. Physiological Plant Pathology. 23:36976.

Heald, F. D., M. W. Gardner, and R. A. Studhalter. 1915. Air and wind dissemination of ascospores of the chesnut blight fungus. Journal of Agricultural Research. 3:493-526. 
Hebard, F. 2005. The backcross breeding program of the American Chestnut Foundation. Journal of the American Chestnut Foundation. 19(2):55-78.

Heiniger, U. and D. Rigling. 1994. Biological control of chestnut blight in Europe. Annual Review of Phytopathology. 32:581-99.

Hillman, B. I., B. T. Halpern, and M. P. Brown. 1994. A viral dsRNA element of the chestnut blight fungus with a distinct genetic organization. Virology. 201(2):241-50.

Hillman, B. I. and N. Suzuki. 2004. Viruses of the chestnut blight fungus, Cryphonectria parasitica. Advances in Virus Research. 63:423-72.

Jaynes, R. A., \& Elliston, J. E. 1978. Control of Endothia parasitica cankers on American chestnut sprouts with hypovirulent strains. In Proceedings of the American Chestnut Symposium. W.L. MacDonald, F.C. Cech, J. Luchok, and C. Smith, eds. WV Univ. Books, Morgantown. 110-114.

Kues, U. and L. A. Casselton. 1992. Fungal mating type genes - regulators of sexual development. Mycological Research. 12: 993-1006.

Lee, J. K., T. A. Tattar, P. M. Berman, and M. S. Mount. 1992. A rapid method for testing the virulence of Cryphonectria parasitica using excised bark and wood of American chestnut. Phytopathology. 82(12): 1454-56.

Linder-Basso, D., J. N. Dynek, and B. I. Hillman. 2005. Genome analysis of Cryphonectria hypovirus 4, the most common hypovirus species in North America. Virology. 1:193203.

Liu, Y. and M. G. Milgroom. 1996. Correlation between hypovirus transmission and the 
number of vegetative incompatibility (vic) genes gifferent among isolates from a natural population of Cryphonectria parasitica. Phytopathology. 86(1): 79-86.

MacDonald, W. L. and D. W. Fulbright. 1991. Biological control of chestnut blight: use and limitations of transmissible hypovirulence. Plant Disease. 75(7):656-61.

McGuire, C. R. E. Marra, and M. G. Milgroom. 2004. Mating-type heterokaryosis and selfing in Cryphonectria parasitica. Fungal Genetics and Biology 41:521-33.

Merkel, H. W. 1905. A deadly fungus on the American chestnut. New York Zoological Society, 10th Annual Report, pp. 97-103.

Milgroom, M. G. and P. Cortesi. 1999. Analysis of population structure of the chestnut blight fungus based on vegetative incompatibility genotypes. Proceeding of the National Academy of Science. 96:10518-523.

Milgroom, M. G. and P. Cortesi. 2004. Biological control of chestnut blight with hypovirulence. A critical analysis. Annual Review of Phytopathology. 42:311-38.

Milgroom, M. G., K. Sotirovski, D. Spica, J. E. Davis, M. T. Brewer, M. Milve, and P. Cortesi. 2008. Clonal population structure of the chestnut blight fungus in expanding ranges in southeastern Europe. Molecular Ecology. 17:4446-58.

Milgroom, M. G., K. Sotirovski, M. Risteski, and M. T. Brewer. 2009. Heterokaryons and parasexual recombinants of Cryphonectria parasitica in two clonal populations in southeastern Europe. Fungal Genetics and Biology. 46(11): 849-54.

Morris, J. T. and J. A. Dodds. 1979. Isolation and analysis of double-stranded RNA from virusinfected plant and fungal tissue. Phytopathology. 69(8):854-58.

Newhouse, A. E., J. E. Spitzer, C. A. Maynard, and W. A. Powell. 2014. Chestnut leaf 
inoculation assay as a rapid predictor of blight susceptibility. Plant Disease. 98(1):4-9.

Newhouse, A. E., L. D. Polin-McGuigan, K. A. Baier, K. E. R. Valletta, W. H. Rottmann, T.

J. Tschaplinski, C. A. Maynard, and W. A. Powell. 2014. Transgenic American chestnuts show enhanced blight resistance and transmit the trait to T1 progeny. Plant Science. 228. 88-97.

Nuss, D. L. 2005. Hypovirulence: Mycoviruses at the fungal-plant interface. Nature 3:63242.

Pavari, A. 1949. Chestnut blight in Europe. Unasylva 3:8-13.

Peever, T. L., Y. Liu, and M. G. Milgroom. 1997. Diversity of hypoviruses and other double-stranded RNAs in Cryphonectria parasitica in North America. Phytopathology. 87(10): 1026-33.

Peever, T. L., Y. Liu, K. Wang; B. I. Hillman, R. Foglia, and M. G. Millgroom. 1998. Incidence and diversity of double-stranded RNAs occurring in the chestnut blight fungus, Cryphonectria parasitica, in China and Japan. Phytopathology. 88(8): 811-17.

Peever, T. L., Y. Liu, P. Cortesi, and M. G. Milgroom. 2000. Variation in tolerance and virulence in the chestnut blight fungus-hypovirus interaction. Applied and Environmental Microbiology. 66(11): 4863-69.

Prospero, S., M. Conedera, U. Heiniger, and D. Rigling. 2006. Saprophytic activity and sporulation of Cryphonectria parasitica on dead chestnut wood in forests with naturally established hypovirulence. Phytopathology. 96(12):1337-44.

Rigling, D., Heiniger, U., Hohl, H. R. 1989. Reduction of laccase activity in dsRNA- 
containing hypovirulent strains of Cryphonectria (Endothia) parasitica. Phytopathology 79:219-23.

Short, D. P. G., M. Double, D. L. Nuss, C. M. Stauder, W. L. MacDonald, and M. T. Kasson. 2015. Multilocus PCR Assays Elucidate Vegetative Incompatibility Gene Profiles of Cryphonectria parasitica in the United States. Applied and Environmental Microbiology. 81(17): 5736-42.

Smart, C. D., W. Yuan, R. Foglia, D. L. Nuss, D. W. Fulbright, and B. I. Hillman. 1999. Cryphonectria hypovirus 3, a virus species in the family Hypoviridae with a single open reading frame. Virology. 265(1):66-73.

Torsello, M. L., D. D. Davis, and B. L. Nash. 1994. Incidence of Cryponectria parasitica cankers on scarlet oak (Quercus coccinea) in Pennsylvania. Plant Disease. 78(3): 313-15.

Van Alfen, N. K., R. A. Jaynes, S. L. Anagnostakis, and P. R. Day. 1975. Chestnut blight: biological control by transmissible hypovirulence in Endothia parasitica. Science. 189(4206): 890-891.

Zamora, P., A. B. Martin, M. Duenas, R. San Martin, and J. J. Diez. 2015. Cryphonectria parasitica isolates of the same vegetative compatibility type display different rates of transfer of CHV1 hypovirus. European Journal of Plant Pathology. 143: 767-77. 


\title{
CHAPTER 2: COMPARISONS OF HOST SUSCEPTIBILITY TO STEM INOCULATIONS OF ISOGENIC VIRULENT AND HYPOVIRULENT (CHV1-INFECTED) STRAINS OF CRYPHONECTRIA PARASITICA AMONG AMERICAN (CASTANEA DENTATA), CHINESE (C. MOLLISSIMA), EUROPEAN (C. SATIVA), AND AMERICAN X CHINESE HYBRID (B2F2, B2F3, B3F2) CHESTNUTS
}

\begin{abstract}
Chestnut blight is a lethal canker disease of several members of the family Fagaceae resulting from infections by Cryphonectria parasitica. This disease was first observed by H.W. Merkel in 1904 in the New York City Zoological Park (Merkel, 1905; Anagnostakis, 1987). Within fifty years of its introduction, C. parasitica had decimated American chestnut (Castanea dentata) populations across its entire 3.6 million hectare range in eastern North America, spreading at a rate of ca. $31 \mathrm{~km} /$ year (Anagnostakis, 1987; Evans and Finkral, 2010).

A less devastating but significant epidemic was observed in Europe on European chestnut (Castanea sativa) beginning around 1949 (Pavari, 1949; Anagnostakis, 1987). However, the emergence of hypovirulence followed shortly behind this epidemic. Hypovirulence of $C$. parasitica results from infections by mycoviruses of the family Hypoviridae, which debilitate the fungus resulting in reduced canker development and sexual/asexual reproduction (Hillman, 1994; MacDonald and Fulbright, 1991). The level of debilitation depends on the genetics of the hypovirus, its fungal host, and the interactions between them (Nuss, 2005).
\end{abstract}

Chestnut host resistance is another factor influencing disease progression and also is the motivation for efforts by The American Chestnut Foundation (TACF) to generate blight-resistant 
hybrids by crossing American chestnuts with resistant Chinese chestnuts (Castanea mollissima) (Hebard, 2005). The objective of this study was to assess variations in host susceptibility to isogenic virulent and hypovirulent (infected with hypovirus Euro7-CHV1) strains of $C$. parasitica among a population of American, Chinese, European, and TACF American x Chinese hybrids (B2F2, B2F3, and B3F2). The plantings are located at West Virginia University's agronomy farm. Artificial stem infections were used to assess host resistance by measuring canker growth and stromata production measurements over a one-year period.

When challenged with virulent strain Weekly, Chinese chestnuts were the most resistant producing an average linear growth of $13.92 \mathrm{~cm}$. Linear growth of all other hosts were not significantly different from American which averaged $17.92 \mathrm{~cm}$. Stromata density also was significantly lower on Chinese $\left(0.16\right.$ stromata $\left./ \mathrm{cm}^{2}\right)$ than all other hosts. Several included analyses found similar levels of resistance to Chinese chestnuts within the TACF hybrid generation B2F2. Climatic influences on C. parasitica's growth and stromata production also were explored. Temperature was positively correlated with changes in growth and stromata density. Hypovirulent Weekly-CHV1 ceased growth during the first two months of the study, yet isolate recovery was possible from canker tissue 9-mo. post-inoculation. Hypovirulent canker linear growth was significantly less than virulent canker linear growth, but few significant differences could be identified among hosts. The findings of this study begin to establish a relationship among the TACF hybrids and other chestnut species relative to host resistance and pathogen virulence. 


\section{INTRODUCTION}

Chestnut blight, caused by the fungal pathogen Cryphonectria parasitica, is a canker disease of members of Fagaceae and especially those in the genus Castanea. Species of Castanea exhibit varying levels of susceptibility to the chestnut blight pathogen (Graves, 1950; Anagnostakis, 1991; Berry, 1960; Clapper, 1952). Among them, Chinese chestnuts (Castanea mollissima) are considered resistant, American chestnuts (C. dentata) are highly susceptible, and European chestnuts exhibit levels of resistance slightly higher than American chestnuts (Graves, 1950).

Unfortunately, the Chinese chestnut cannot fill the role the American chestnut once had in eastern North American as a fast-growing, straight-boled, rot-resistant, keystone species. The Chinese chestnuts are more suitable to orchard settings given their spreading growth form (Schlarbaum et al., 1992). The characteristics of Chinese do not compete with that of the American, and therefore, there has been great interest in the generation of a blight-resistant American chestnut for restoration purposes.

Efforts to incorporate the blight resistance of Chinese chestnut into highly susceptible American chestnuts began as early as 1930, and many generations of such hybrids exist today (Hebard, 2005). Despite this extensive work, the recent American $x$ Chinese hybrid generations have yet to be formally ranked among the other chestnut species. A more recent development utilized transgenic technologies to develop American chestnuts that exhibit levels of resistance higher than even that of Chinese chestnut due to the incorporation of an oxalate oxidase gene from wheat (Zhang, 2013; Newhouse et al., 2014). Both of these efforts provide hope for the restoration of American chestnut. 
In addition to host resistance, the virulence of $C$. parasitica is also a factor which determines host survival. Around 1953, Biraghi noticed "spontaneously healing" cankers on European chestnuts (C. sativa) in Italy. A mycovirus of the family Hypoviridae was later found within these hypovirulent strains and thus named Cryphonectria hypovirus (CHV). More than half a century later, a total of four hypoviruses have been described from hypovirulent strains found in Europe and North America. Each is genetically different and characterized as having varying effects on C. parasitica (Heiniger and Rigling, 1994; Milgroom and Cortesi, 2004; Hillman, 1994; Smart et al., 1999; Peever et al., 1997; Enebek, 1992). Generally, hypovirulent strains of $C$. parasitica have attenuated growth and sexual/asexual reproductive capacity, which result in reduced canker development and dissemination. These characteristics vary based on hypovirus and host genetics as well as the interactions between them (MacDonald and Fulbright, 1991). A formal investigation of host susceptibility of chestnut species to these hypovirulent strains of $C$. parasitica has not been conducted.

The objective of this study was to implement a comparative and comprehensive study of host susceptibility to Cryphonectria parasitica among American, Chinese, European, and American x Chinese hybrid (B2F2, B2F3, B3F2) chestnuts when direct-stem inoculated with one of two strains: a virulent C. parasitica strain named Weekly (EU15; vic genotype 2211-11) and an isogenic hypovirulent strain Weekly Euro7-CHV1. 


\section{MATERIALS AND METHODS}

\section{Study location and description}

The chestnut community for this study is located at the West Virginia University (WVU) Agronomy Farm (39³9'36.52"N and 7954'13.79"W) in Morgantown, WV. In 2006, six plots with a total of 900 trees including American (181), Chinese (189), European (154), and American $x$ Chinese hybrid (B2F2 (82), B2F3 (160), B3F2 (134)) chestnuts were planted randomly in rows on a $2.13 \times 6.1$ meter spacing in an orchard setting.

Three plots $(1,4$, and 5), physically separated by $0.3-0.8 \mathrm{~km}$, were used for this study (Figure 2.1). The plots are at an elevation of approximately $366 \mathrm{~m}$. The three plots were planted in a randomized, complete-block design. Soil surveys reveal the profiles to be composed largely of silt loam and lily loam with slopes ranging from $3-15 \%$.

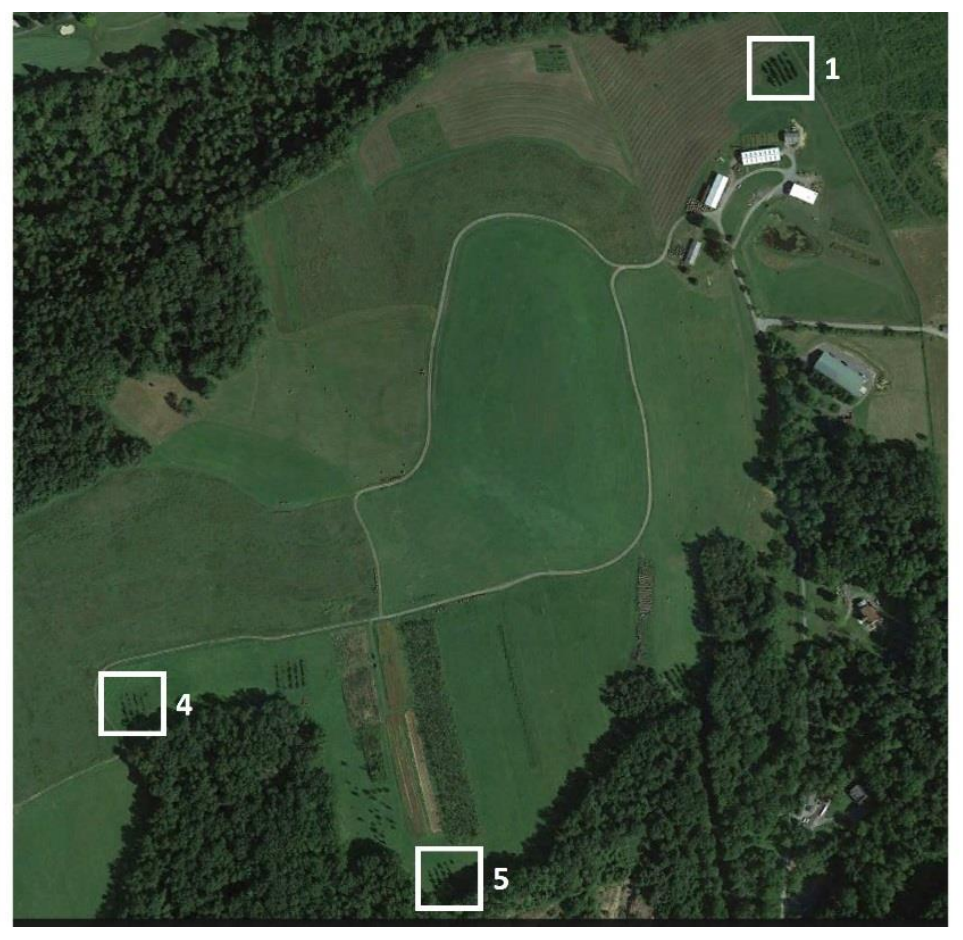

Figure 2.1: Aerial map of WVU Agronomy Farm with plot locations 
For each chestnut species or hybrid, the populations are comprised of trees from various parentages. Ideally, the parentages would be equally represented within the populations, but the study's selection requirements (described below) and the survivorship of previously infected stems (natural and intentional) restricted equal representation.

\section{Experimental design and treatments}

Chestnut trees within plots 1,4 , and 5 were selected based on having $a \geq 140 \mathrm{~cm}$ stem section with a minimum diameter of $\geq 3 \mathrm{~cm}$ and the absence of any potentially lethal blight infections that might have girdled the stem during the time of the study. Three inoculation sites were designated on each stem in areas that were generally free of bud scars, branches, rhytidome, and other potentially confounding plant anatomical features which can affect canker development (Hebard, personal communication).

Following the inventory, 111 trees were randomly selected from those that met the above criteria. European chestnut was the most limiting population with only 10 suitable trees. Each of the other chestnut species and hybrids were represented by 20 or more trees, except for B3F2 hybrids, which had 18 individuals (Table 2.1).

Table 2.1: Total trees by chestnut species or TACF hybrid generation

\begin{tabular}{|c|c|}
\hline Species/Hybrid & Total Trees \\
\hline American & 20 \\
\hline B2F2 & 22 \\
\hline B2F3 & 21 \\
\hline B3F2 & 18 \\
\hline Chinese & 20 \\
\hline European & 10 \\
\hline Total & 111 \\
\hline
\end{tabular}


$\underline{\text { Isolate selection and vic genotyping }}$

Isolates used in this study were selected from the culture collection of $C$. parasitica isolates maintained at WVU. These isolates had been stored on PDA slants at $4^{\circ} \mathrm{C}$.

An excised stem assay was used to compare a selection of $C$. parasitica strains to identify isolates that exhibited moderate virulence with the intention of selecting an isolate which would maximize survival of field tested trees through 1-year post-inoculation. Several of the isolates included in this assay have not been previously assessed with regard to their virulence, while others, including isolate EP 155, have been well characterized and, as such, served as a reference. Stems utilized in this assay were cut from a wild population of American chestnuts in the Savage River State Forest in Maryland.

The excised stem assay procedure as described by Elliston (1978) was conducted with the following exceptions. Stems were incubated at ambient room temperature $\left(c a .21^{\circ} \mathrm{C}\right)$ in the dark for approximately five weeks after which cankers were measured and reisolations were conducted for fungal isolate confirmation. The stem assay results are shown in Figure 2.2. One isolate designated Weekly exhibited a moderate level of virulence among the tested isolates and therefore, was selected for this study. 


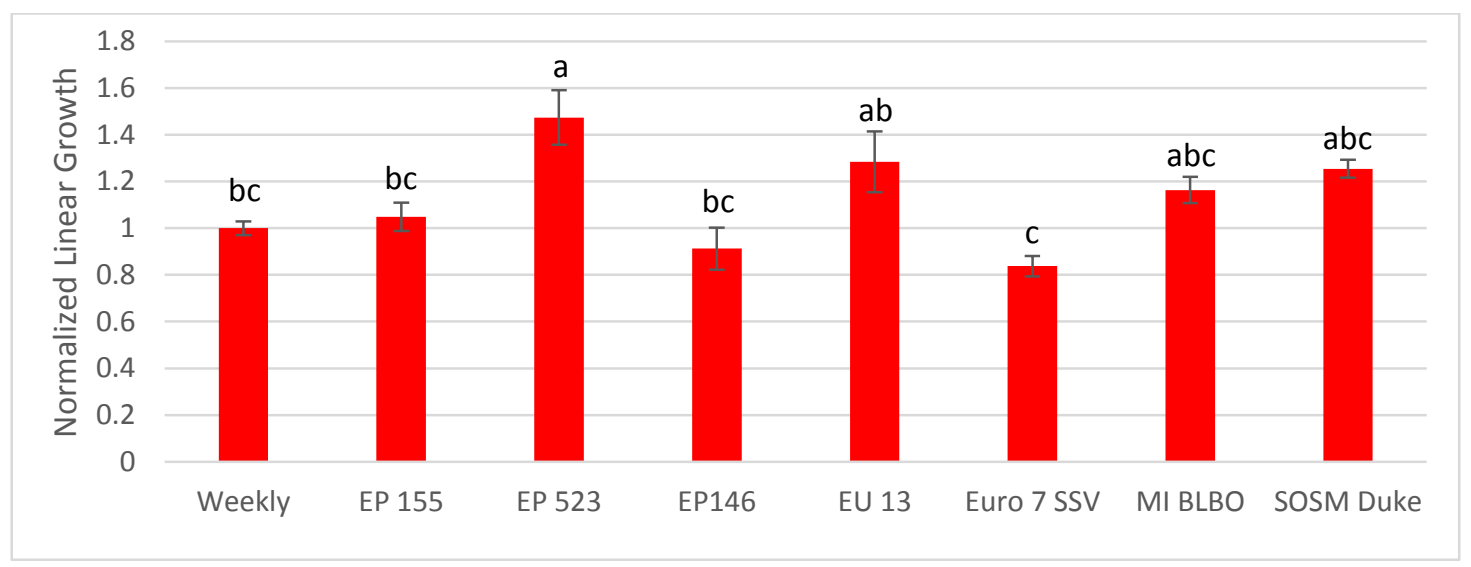

Figure 2.2: Excised stem assay results for selected virulent $C$. parasitica isolates; each measurement was normalized by dividing the value by the overall average linear growth for Weekly inoculations

\section{CHV1 hypovirus transmission}

A CHV1 virus from the Euro7 background was selected as the hypovirus for this study given its level of characterization and the availability of potential donor isolates in the WVU $C$. parasitica collection (Heiniger and Rigling, 1994; Milgroom and Cortesi, 2004). CHV1 viruses commonly reduce the pigmentation of $C$. parasitica resulting in a stark white colony. This feature aids in the visual confirmation of hypovirus infection.

The generation of the isogenic Weekly-CHV1 strain was completed via transmission of hypovirus from available Euro7-CHV1 strains. DNA isolation and vic genotyping as described in Short et al. (2015) were performed to assess the potential for vegetative compatibility among available CHV1 strains and Weekly. This PCR method utilizes sequence-specific primers developed for the two possible alleles for each of the vic loci. Given the primer specificity and the different size amplicons, PCR products of an unknown vic genotype and a known control 
can be visualized simultaneously by gel electrophoresis for comparison to distinguish vic genotypes (Short et al. 2015).

The CHV1 donor isolates were selected from available EU-tester hypovirulent isolates, which were the result of intentional attempts to transmit the Euro7-CHV1 hypovirus via hyphal anastomosis to the 64 EU-tester strains by co-culturing with a collection of CHV1-containing isolates (HVA). The resolved genotypes of these EU-tester strains represent the 64 possible vic genotypes when considering the 6 vic genes The EU-tester strains commonly are used for culture-based vic genotyping (Cortesi and Milgroom, 1998).

Because vegetative compatibility regulates hypovirus transmission, Weekly's genotype was compared with the available CHV1 isolates to choose exact or near matches (Table 2.2). Given the lack of known exact matches in the Euro7-CHV1 C. parasitica collection, isolates with vic genotypes comprised of only one allelic difference were selected for Weekly $\mathrm{x}$ hypovirulent donor pairing assays (described below) for hypovirus transmission.

Table 2.2: Vegetative incompatibility genotypes of selected virulent and hypovirulent isolates

\begin{tabular}{|c|c|}
\hline Isolate & vic genotype \\
\hline \hline Weekly (V) & $2211-11$ \\
\hline EU9-HVA19 (CHV1) & $2111-11$ \\
\hline EU13-HVA32 (CHV1) & $1211-11$ \\
\hline EU21-HVA36 (CHV1) & $2211-12$ \\
\hline EU28-HVA37 (CHV1) & $2212-11$ \\
\hline EU46-HVA21 (CHV1) & $2221-11$ \\
\hline
\end{tabular}


Isolate co-culturing for hypovirus transmission was conducted on PDA + tween (39 g

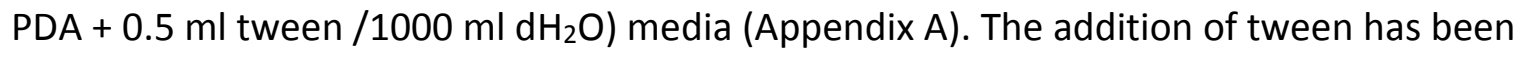
observed to create growth conditions that promote hyphal anastomosis among compatible strains (M. Double, personal communication). Weekly and hypovirulent isolates were transferred to the assay plate in close proximity to the upper edge and each other as shown in Figure 2.3.

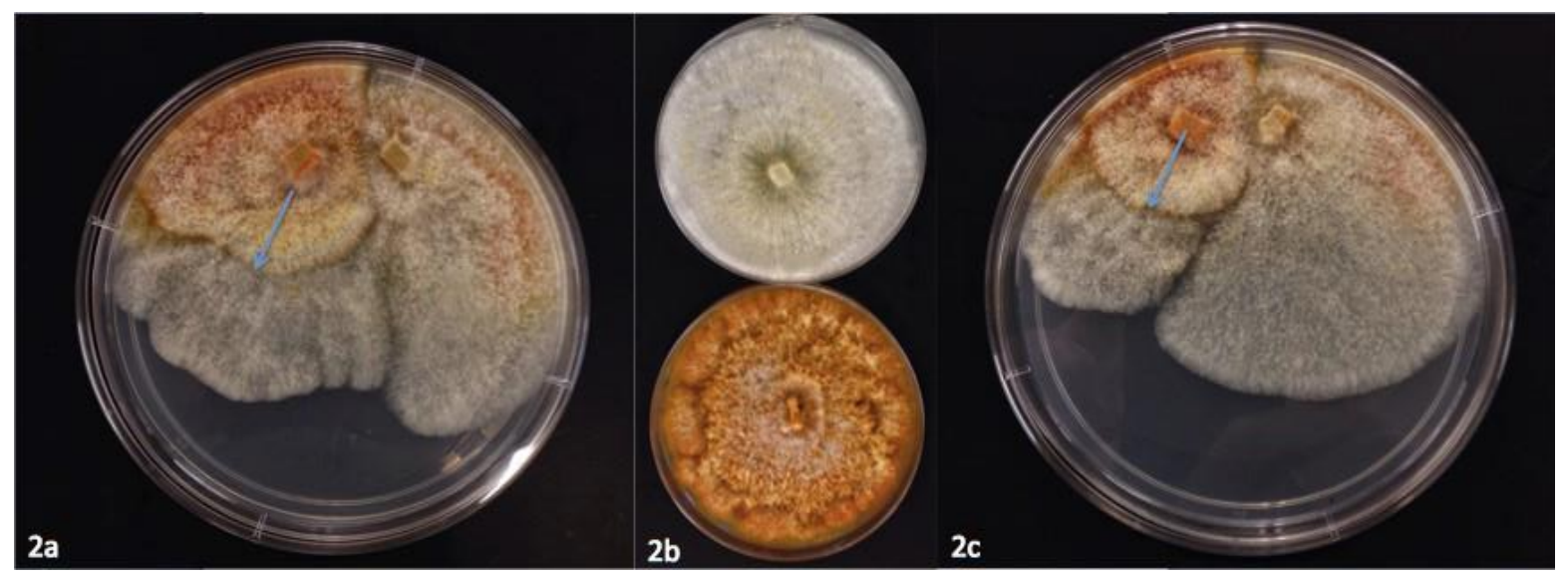

Figure 2.3 (2a): Weekly x CHV1 isolate pairing with hypovirus transmission; (2b): Weekly-CHV1 (top) and Weekly virulent (bottom); (2c): Weekly paired with Weekly-CHV1

Potential transmission of the hypovirus was observed based on a distinct shift in the morphology of the virulent strain (Figure $2.3(2 a)$ ). The leading edge of the potential Weekly isolate that had acquired the CHV1 hypovirus was then transferred to a new PDA plate to make morphological comparisons to Weekly (Figure 2.3 (2b)). Weekly-CHV1 was then paired with the virulent Weekly strain to observe hypovirus transmission to the virulent strain (Figure 2.3 (2c)). This transmission should readily happen as the two isolates have matching genotypes. Distinct 
differences in growth between Weekly and the potential Weekly-CHV1 isolate, isogenic virulent-hypovirulent hypovirus transmission, and the confirmation of the Weekly genotype (2211-11) among Weekly-CHV1 isolates justified the following dsRNA extraction assay.

The generation of Weekly-CHV1 occurred when paired with two donating isolates: EU 28-HVA37 and EU46-HVA21. The Weekly-CHV1 from the EU46-HVA21 donor was chosen for use in the study. The generation of a Weekly-CHV3 isolate was also attempted through the same pairing assay described above using a number of CHV3 containing isolates, but no transmission was observed across all pairing assays. Therefore, Weekly-CHV3 was removed from the original experiment's design.

dsRNA extraction

To confirm the presence of dsRNA associated with suspected Weekly-CHV1 strains, a modified dsRNA isolation protocol previously described by Morris and Dodds (1979) (Method 1) was used with some modifications. Samples were prepared in liquid nitrogen and final samples were dissolved in $20 \mu \mathrm{l}$ of $22 \mathrm{mM}$ Tris, $22 \mathrm{mM}$ boric acid, $250 \mu \mathrm{M} \mathrm{Na2,} \mathrm{EDTA} \mathrm{buffer} \mathrm{with} \mathrm{10 \%}$ sucrose and $0.001 \%$ bromophenol blue. One percent agarose gels containing $40 \mu$ l ethidium bromide per $100 \mathrm{ml}$ agarose were used for visualization.

$\underline{\text { Inoculum preparation and inoculation procedure }}$

Weekly virulent and Weekly-CHV1 isolates were incubated on PDA for approximately 10 days at approximately $20.0^{\circ} \mathrm{C}$ under a $16-8 \mathrm{hr}$ light-dark cycle. Inoculum plugs were made using 
a $1 \mathrm{~cm}$ leather punch along the growing edge of the fungal colony. For negative controls, a sterile $1 \mathrm{~cm}$ leather punch was used to generate identically sized agar plugs from sterile PDA.

Using an inoculation method previously described by Jaynes and Elliston (1978), a hammer and $1 \mathrm{~cm}$ leather punch were used to create an inoculation hole by removing the bark and exposing the vascular cambium for each of three inoculation sites on each test tree included in the study. Inoculations proceeded as each tree received a Weekly virulent, WeeklyCHV1, and a negative control plug. Inoculum placement was randomized on each stem, and once inoculated, tape was applied to minimize drying and maximize fungal establishment. All inoculations were made on June 1, 2015.

\section{Canker linear growth and stromata estimates}

Canker measurements were made every two months for one year. The edge of the canker was marked with a Sharpie then the length and width of the canker was measured. For each canker, the linear growth was calculated by the following formula: Linear Growth (LG)= (length+width)/2. Rate of canker expansion was calculated by the following formula: Linear Growth $=\left(L G_{\text {period- }-\mathrm{B}}-\mathrm{L} \mathrm{G}_{\text {period }-\mathrm{A}}\right)$.

Stromata counts were performed using a transparent $1 \times 1 \mathrm{~cm}$ grid as a means of estimating sporulation. Across the grid, $75 \%$ of the sections were randomly blocked out such that $25 \%$ of the sections were left open to count stroma. This grid was placed over the canker and stromata were counted within the open grid cells. Given the intensive nature of this measurement, approximately $50 \%$ of the study trees were subjected to this measurement. This $25 \%$ estimation was then adjusted to a $100 \%$ and divided by the canker area 
$\left(=\mathrm{pi}^{*}(1 / 2 *\right.$ Length $) *(1 / 2 *$ Width $\left.)\right)$ to present stromata count as stromata/ $\mathrm{cm}^{2}$. This conversion was completed to represent the relative number of stromata present by controlling for the influence of canker area on the estimate.

In the event of tree death, linear growth measurements and stromata counts were discontinued as it was observed that the pathogen was then able to grow largely unimpeded and sporulate at significantly higher rates. As trees died during the course of the study, the expanding fungal colonization was visually confirmed by scraping a region of bark at the proximal margin of the suspect canker to confirm green, living tissue below the margin and necrotic tissue above.

\section{Visual assessment of cankers}

At the end of the study, an ordinal ranking system was employed to assess each canker. The ranking system included ranking from 1-5, which qualified the amount of callus tissue and stromata present for each canker (Table 2.3). This method served to quickly assess overall host response to C. parasitica infections.

Table 2.3: Rank descriptions for visual assessment of cankers

\begin{tabular}{|c|c|}
\hline Rank & Description \\
\hline 1 & Callus, no stroma \\
\hline 2 & Callus, stroma present \\
\hline 3 & Callus, abundant stroma \\
\hline 4 & No callus, abundant stroma \\
\hline 5 & Dead \\
\hline
\end{tabular}


$\underline{\text { Tree diameter measurements and callus estimation }}$

Prior to inoculation and at the end of the study period, the diameter of tree at each of the three inoculation sites and the diameter of the stem at breast height ( $1.3 \mathrm{~m}$ above ground) was measured with a diameter tape. Diameter increase resulting from the development of swollen callus surrounding the inoculation (CDI) was estimated using $\left[C D I=\left(D_{2}-A D I\right)-D_{1}\right]$ with $D_{1}$ as the starting diameter of the inoculation site of interest, $D_{2}$ as the ending diameter of that site, and $A D I$ as the average diameter increase across all measurements on that stem.

\section{Environmental measurements}

Climate data was obtained from the PRISM Climate Group for the WVU Agronomy Farm area. Given the 2-month measurement periods used in this study, monthly temperature and precipitation were averaged for the each two-month period to estimate the average temperatures and precipitation between measurements. These data served to help elucidate how varying conditions influenced growth and sporulation changes over the course of the oneyear study.

\section{Cryphonectria parasitica reisolation and isolate confirmation}

Nine months post-inoculation approximately of $10 \%$ of cankers resulting from Weekly and Weekly-CHV1 were sampled using a microsampler to excise bark plugs from the canker's outer edge. These plugs were washed for 14 minutes in a 1:10 commercial bleach-water solution for surface sterilization then placed onto glucose yeast-extract (GYE) agar amended with streptomycin sulfate and tetracycline hydrochloride antibiotics to permit fungal isolations 
(Appendix A). Resulting C. parasitica isolates were subcultured, grown on potato-dextrose

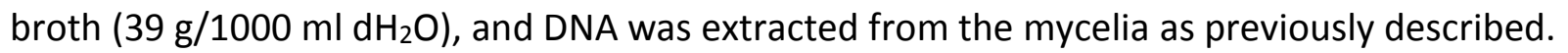
Vic genotyping permitted confirmation of Weekly's vic genotype for isolates recovered from cankers after 9 months. The use of the dsRNA extraction described above served as the means of confirming the hypovirulence of isolates recovered from hypovirulent cankers. This extraction was completed on a subset of isolates, and morphological comparisons served as a means of confirming the hypovirulence of isolates not included in the dsRNA extraction.

\section{Analyses}

All analyses were performed using JMP ${ }^{\circledR} 10$ software (SAS Institute, Inc., Cary, NC, USA). Mean comparisons served as the main analysis utilized to discern differences among host backgrounds, inoculum sources, and periods throughout the study. To test these, Tukey's HSD $(\alpha=0.05)$ was selected to make these comparisons among $>3$ dependent variables, and Students $t(\alpha=0.05)$ for analyses with $\leq 3$ dependent variables. Values of significance are found within each figure and were calculated with the previously described analyses. For climate influences on the change in growth and stromata production, one way ANOVA tests were performed. Pairwise comparisons were conducted for host mortality using a Kaplan-Meier survival analysis ( $\alpha=0.003)$. 


\section{RESULTS}

Inoculations for all hosts were successful except for three failed inoculation for virulent Weekly and three Weekly-CHV1 inoculations which became infected with a wild strain of $C$. parasitica. Mortality limited several sample populations, largely American, and therefore, the following results only represent measurements taken on trees living at the end of the study. Analyses that included dead trees are noted below.

\section{Linear growth}

Linear growth serves as a direct measure of the ability of $C$. parasitica to colonize a host's tissue around the inoculation. Linear growth was measured every two months for a year. Inoculations with virulent Weekly resulted in comparable average linear growth measurements across all hosts with the exception of Chinese which had a significantly lower average linear growth than most other hosts $(13.92 \mathrm{~cm}$ ) (Figure 2.4). Surprisingly, cankers on TACF hybrid generation B3F2 trees had the highest average linear growth $(20.02 \mathrm{~cm})$, but this value was not significantly greater than American, B2F2, B2F3, or European chestnuts, which had linear growth measurements of $17.92,18.56,18.98$, and $19.84 \mathrm{~cm}$, respectively. Also, American chestnuts had linear growth measurements that were also similar to Chinese chestnuts. 


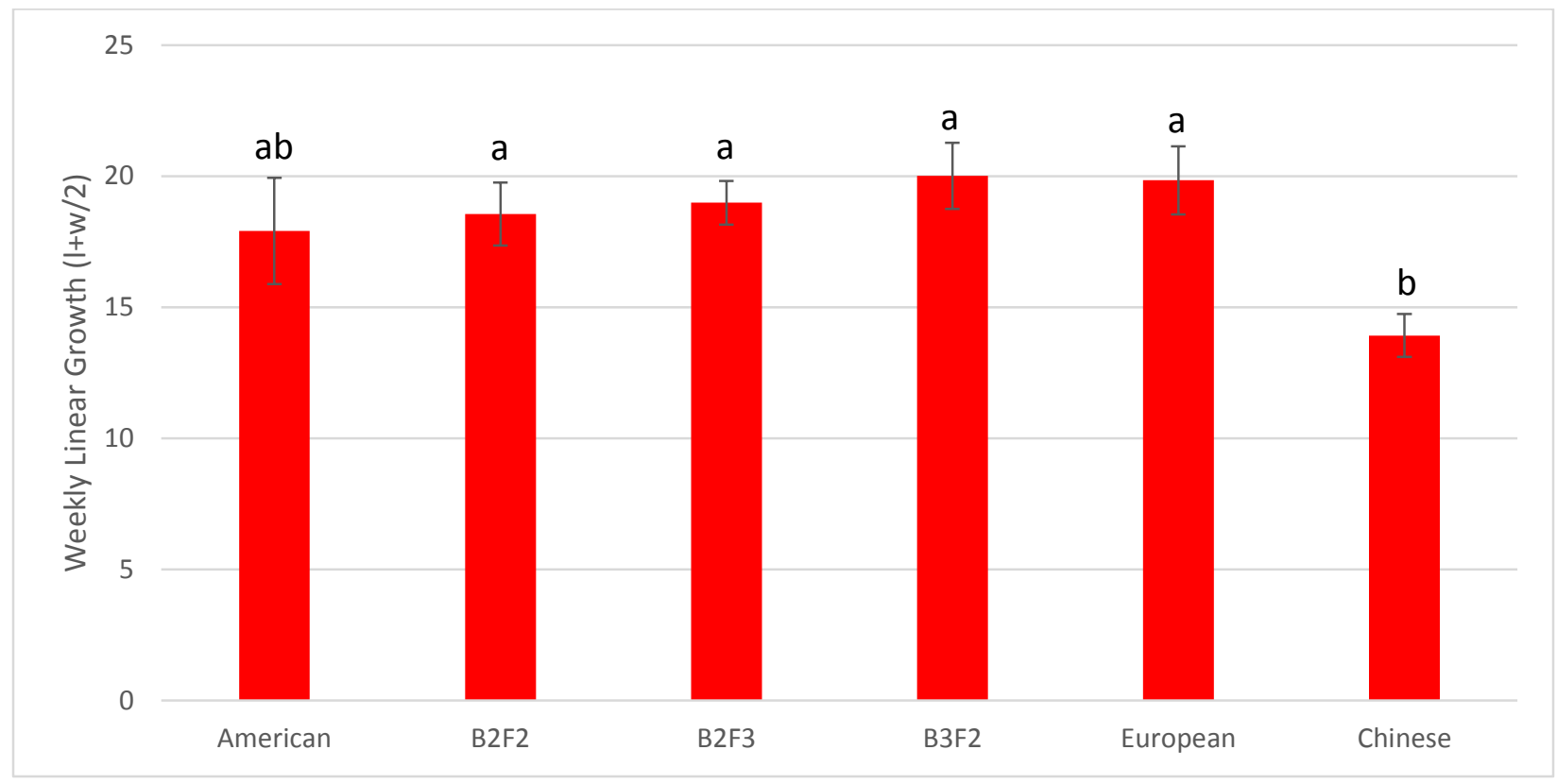

Figure 2.4: Average linear growth $(1+w / 2)$ for Weekly inoculations for each host background; error bars represent +/- $1 \mathrm{SE}$

All inoculations with the Weekly strain infected with the Euro7-CHV1 hypovirus (Weekly-CHV1) also produced cankers, but canker expansion ceased during the first eight weeks. Three of 111 cankers expanded in subsequent months on American chestnut, but virulent isolates with vic genotypes not matching Weekly-CHV1 were recovered from these infections while all others resulted in hypovirulent Weekly-CHV1 (Table 2.6). Therefore, measurements for the three wild-type infections were removed from the study. Since the canker margins were marked after the first eight weeks, a visual assessment was employed for the remaining measurement periods to check for continued growth. Given the lack of continued growth, no additional measurements were taken. 
The following data represent the amount of linear growth that was measured after the first eight weeks and did not change for the duration of the study. Average linear growth for cankers on European chestnut $(4.97 \mathrm{~cm})$ was less than that measured for Weekly-CHV1 cankers on all other hosts (Figure 2.5). Cankers on Chinese chestnut had the most linear growth (7.63 $\mathrm{cm})$, but that value was not statistically different from measurements for cankers on American, B2F2, B2F3, and B3F2 which had linear growth measurements of $6.65,6.74,7.73$, and $6.81 \mathrm{~cm}$ respectively.

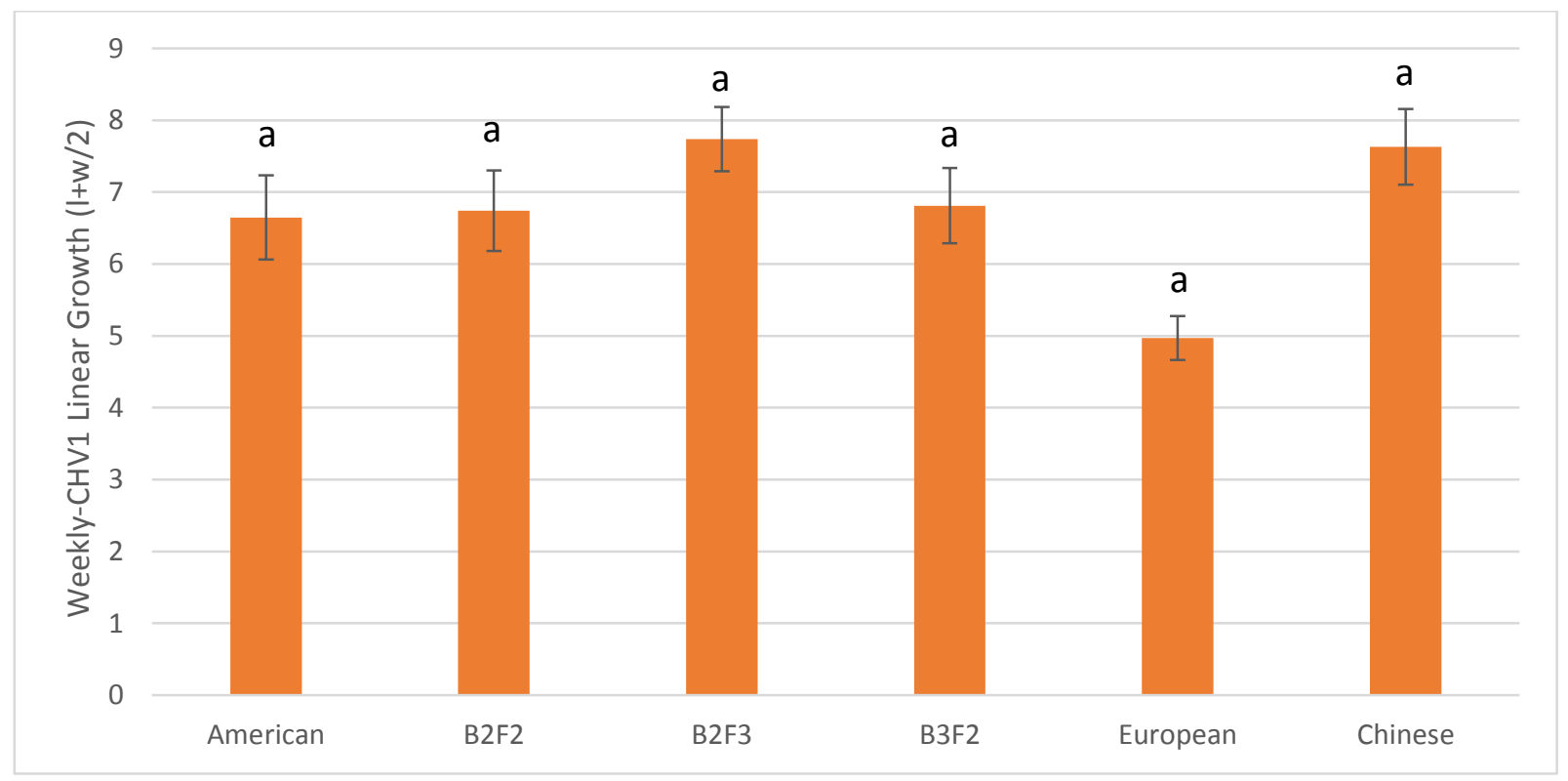

Figure 2.5: Average linear growth $(I+w / 2)$ for Weekly-CHV1 inoculations for each host background; error bars represent +/- 1 SE 
Linear growth of cankers averaged for all hosts resulted in significantly higher averages for virulent Weekly $(17.92 \mathrm{~cm})$ compared to Weekly-CHV1 $(6.93 \mathrm{~cm})$, which produced cankers only slightly larger than the linear growth measured for sterile PDA control $(3.44 \mathrm{~cm})$ (Figure 2.6). In this case, the control has linear growth values which represents the inoculation hole created at the beginning of the study and any response of the tree to wounding.

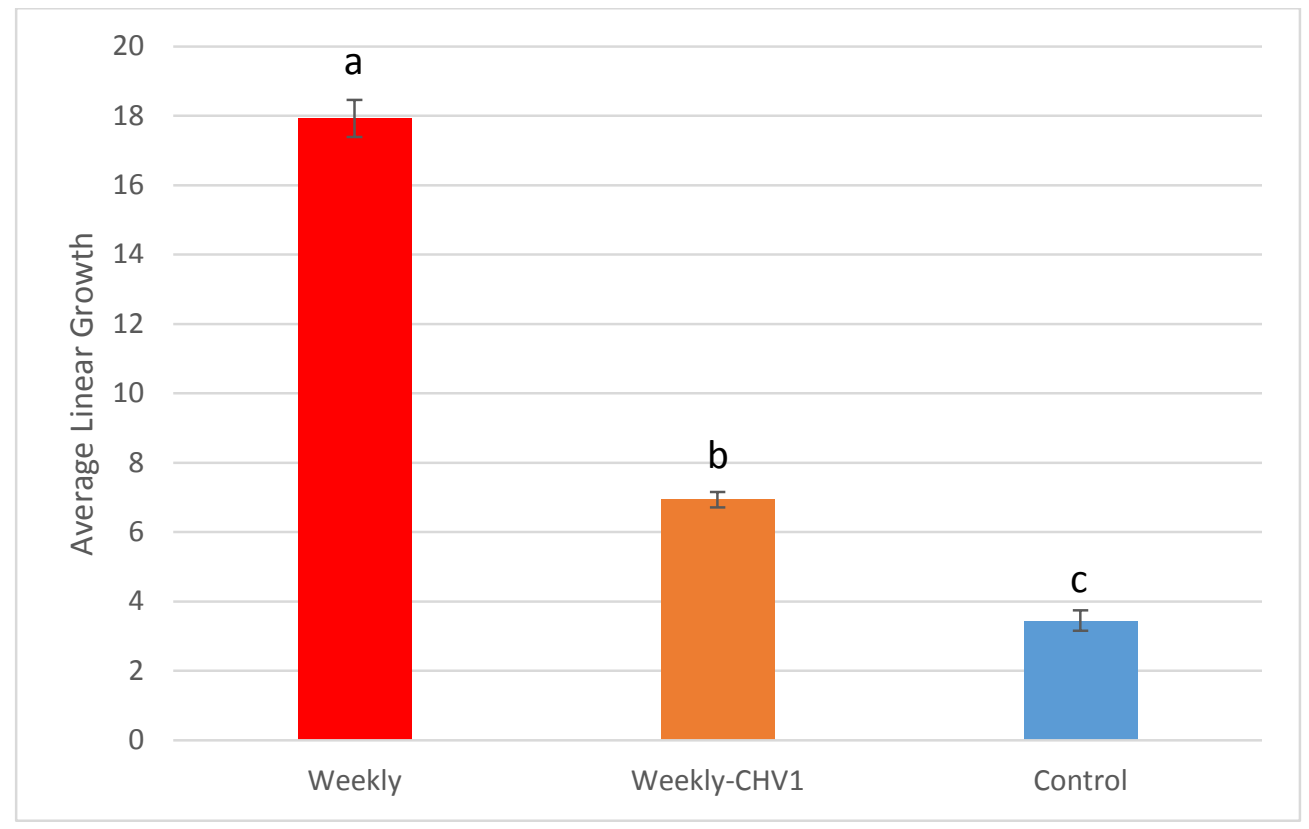

Figure 2.6: Average linear growth $(1+w / 2)$ for Weekly, Weekly-CHV1, and control inoculations; error bars represent +/- $1 \mathrm{SE}$

\section{Repeated measure of linear growth analysis}

Given the lack of many significant differences in linear growth at the end of the study, a repeated measures ANOVA was employed to analyze each linear growth measurement recorded for all six measurement periods. Using this method, each measurement of linear growth can influence the overall analysis. The trends analyzed here show instances of 
disproportionate changes in linear growth among cankers on the different hosts between measurement periods. The repeated measures ANOVA test captures such differences and therefore, is an appropriate analysis. The results indicate that linear growth on B2F2 and European chestnuts are not significantly different than linear growth measurements for Chinese chestnut. The remaining host backgrounds show significantly similar ANOVA values to American, B2F3, and B3F2 chestnuts (Figure 2.7).

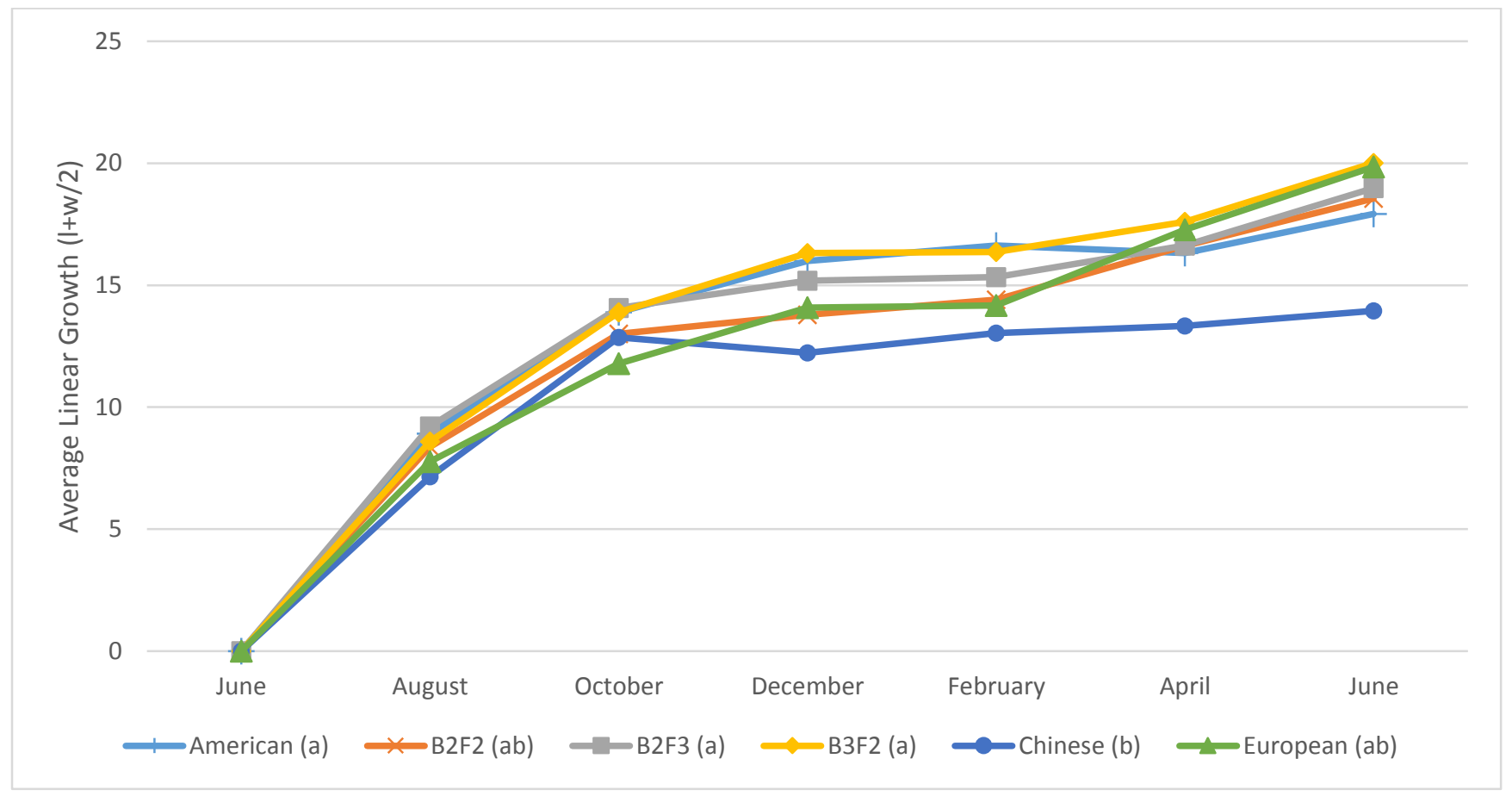

Figure 2.7: Average virulent Weekly linear growth $(1+w / 2)$ for each host background by measurement period; significance values located in the legend represent results from a repeated measures ANOVA 


\section{$\underline{\text { Rate of canker expansion }}$}

Given the lack of subsequent growth for Weekly-CHV1 initiated infections, the following rate of canker expansion analysis was completed for virulent Weekly inoculations. Here, the data were represented by the amount of linear growth between measurement periods ( 60 days/period). Rates of canker expansion varied by measurement period and host background (Figure 2.8), and overall averages varied for each measurement period (Figure 2.9). For virulent Weekly, the highest rate of canker expansion averaged for all hosts was $8.4 \mathrm{~cm} /$ period during the months of June - August (Figure 2.9). The lowest rate of canker expansion was observed during the December - February period of an average of $6.35 \mathrm{~cm} /$ period. There was no significant difference among October to December (1.67 cm/period), February to April (1.59 $\mathrm{cm} /$ period), and April to June $(2.01 \mathrm{~cm} /$ period $)$.

Cankers expanded at a similar rate for each host regardless of measurement period. Chinese chestnut had significantly lower rates of canker expansion than all other host except European for June-August, but in all other time periods, no host was significantly different from the rest. The highest average rate of canker expansion occurred from June-August for TACF hybrid generation B2F3 $(9.22 \mathrm{~cm})$ while the lowest was from December - February on European chestnuts $(0.33 \mathrm{~cm})$. The average rate of canker expansion for the entire duration of the study also was lower for Chinese at an average of $2.77 \mathrm{~cm}$ than all other hosts. 


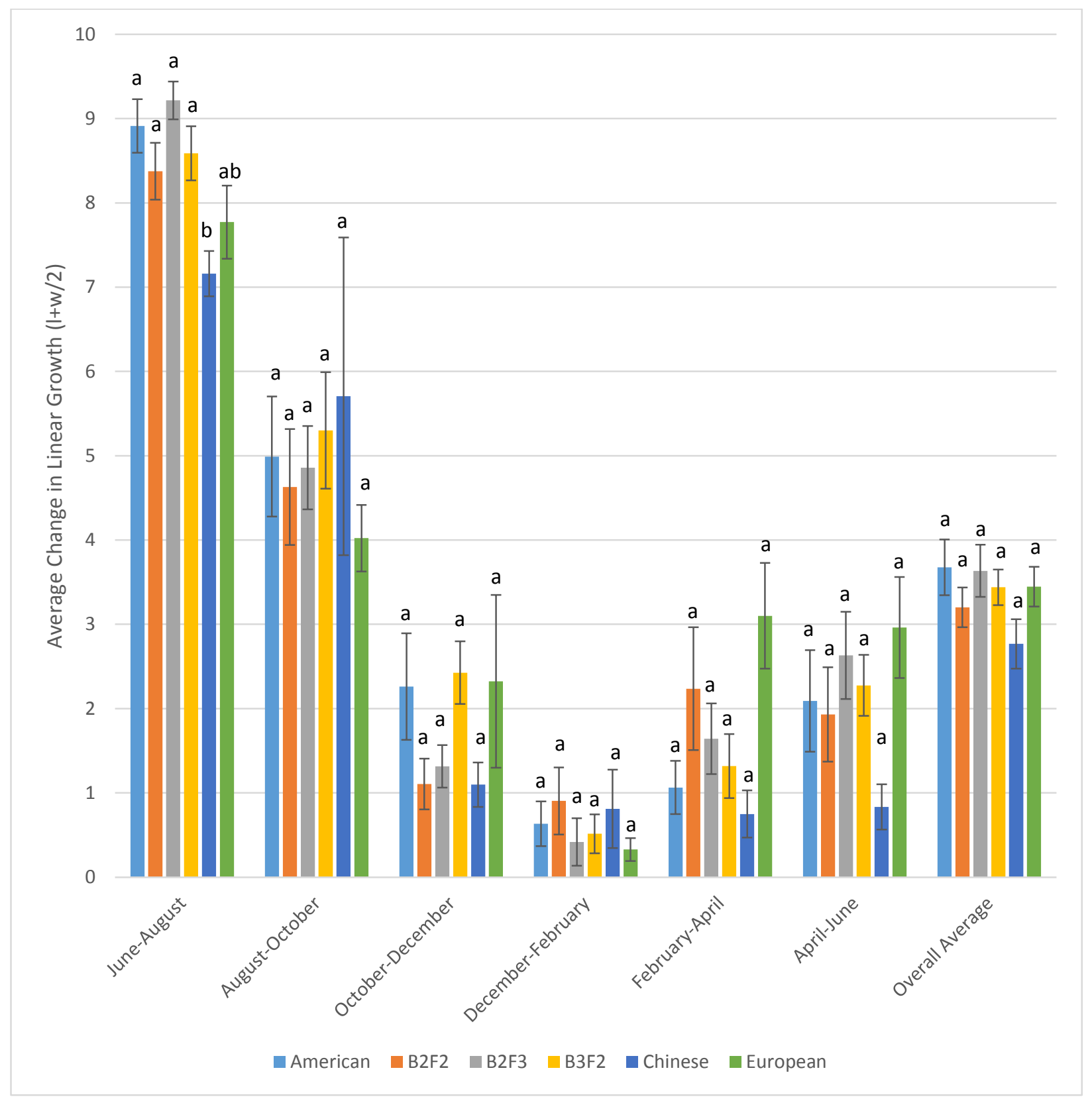

Figure 2.8: Average virulent Weekly rate of canker expansion (cm/period) for each host background across the six measurement periods of this study; error bars $=+$ - $1 \mathrm{SE}$ 


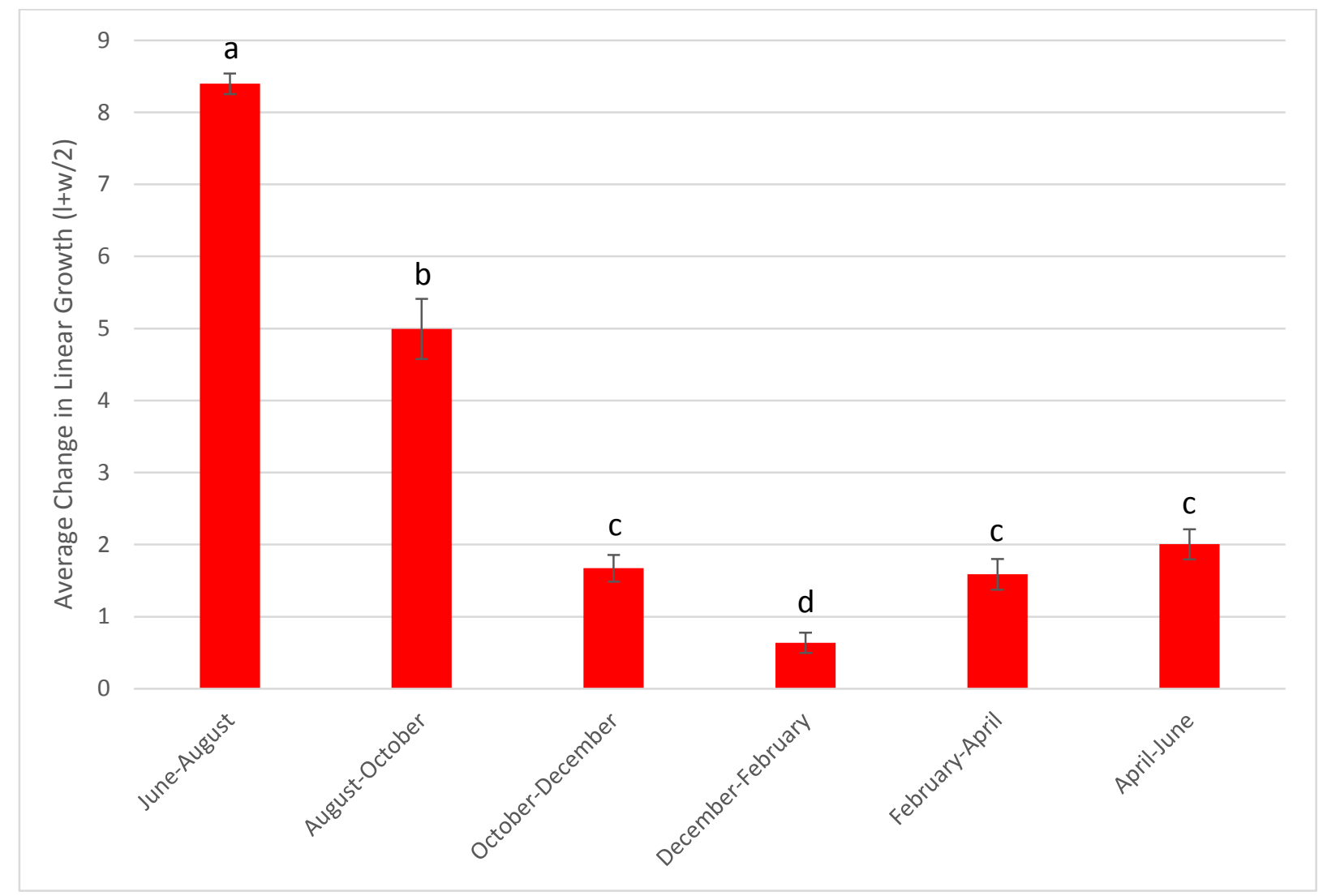

Figure 2.9: Overall average rate of canker expansion for virulent Weekly for the six measurement periods of this study

\section{$\underline{\text { Stromata counts }}$}

Stromata density analyses were performed on approximately $50 \%$ of Weekly cankers in this study due to the sampling limitations mentioned above. Weekly-CHV1 cankers failed to sporulate during this study, and therefore, no analyses were performed. Average stromata density estimates for virulent Weekly cankers on individual host backgrounds revealed Chinese permitted the lowest density with an average of 0.3 stromata $/ \mathrm{cm}^{2}$. The highest density of stromata was observed on B3F2 chestnuts with an average of 0.7 stromata/ $\mathrm{cm}^{2}$ (Figure 2.10). All other hosts were not significantly different from Chinese and B3F2 chestnuts. 


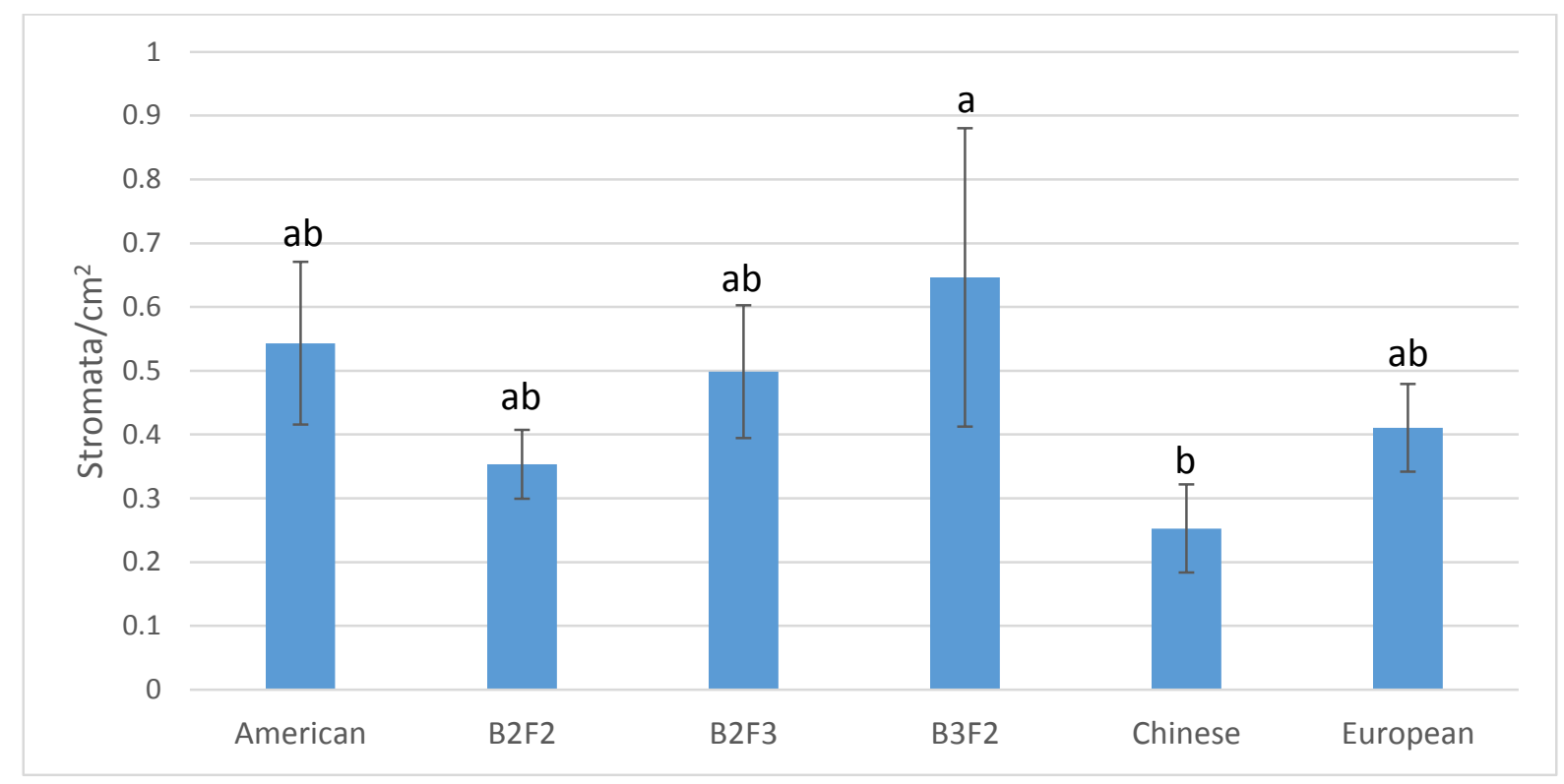

Figure 2.10: Average stromata/cm2 for individual host backgrounds; error bars represent +/- $1 \mathrm{SE}$

The presence of stromata fluctuated in density among measurement periods (Figure 2.11; Figure 2.12). The high density of stromata were counted during the October regardless of host background with the highest count of $1.2 \mathrm{stroma} / \mathrm{cm}^{2}$ on B2F3 (Figure 2.12). The lowest density was recorded during February on Chinese with 0.1 stroma/ $/ \mathrm{cm}^{2}$ (Figure 2.11). A repeated measures of ANOVA was not performed on these data due to the high variability of the data. 


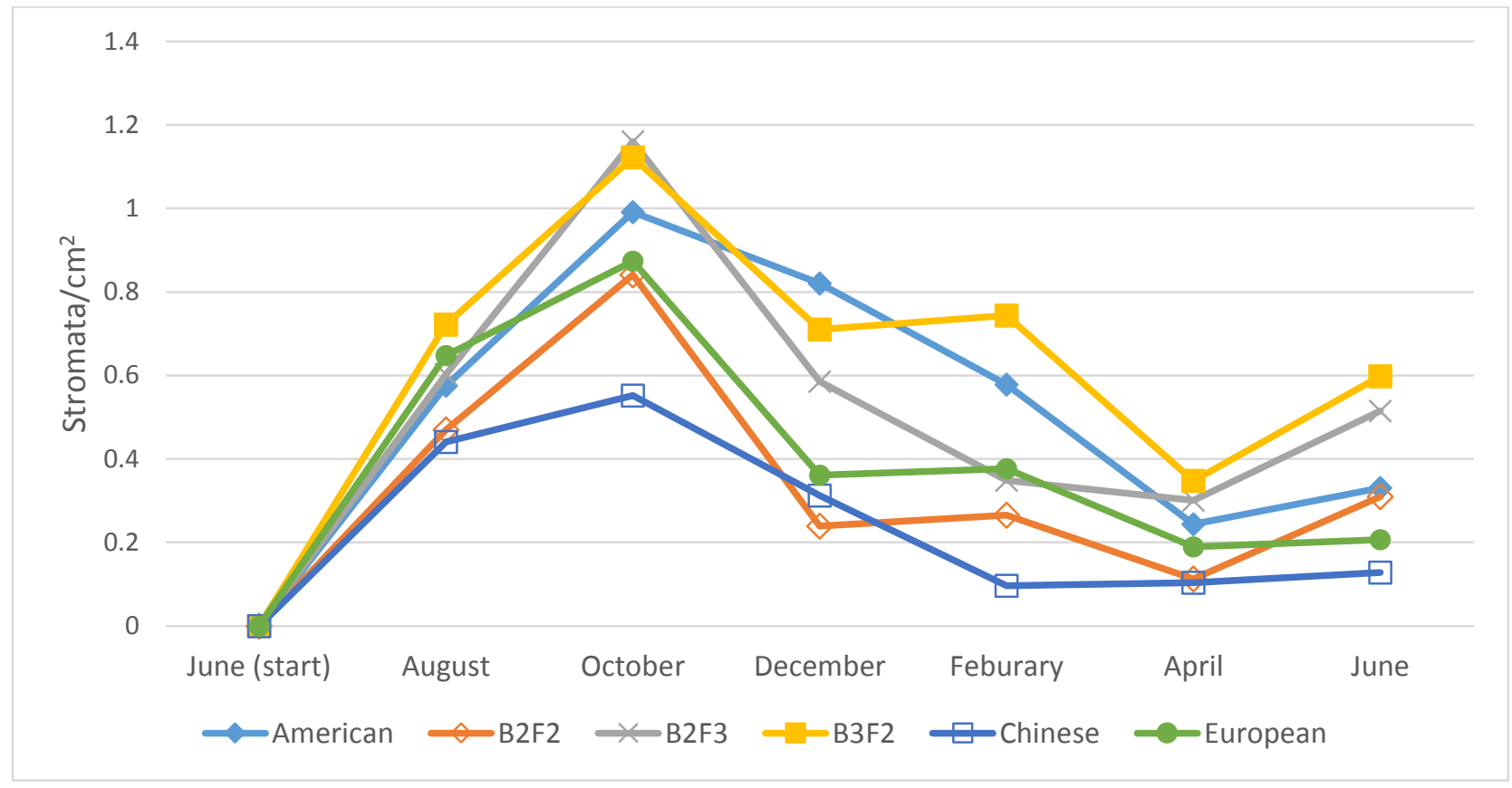

Figure 2.11: Average stromata/ $\mathrm{cm}^{2}$ over time for individual host backgrounds

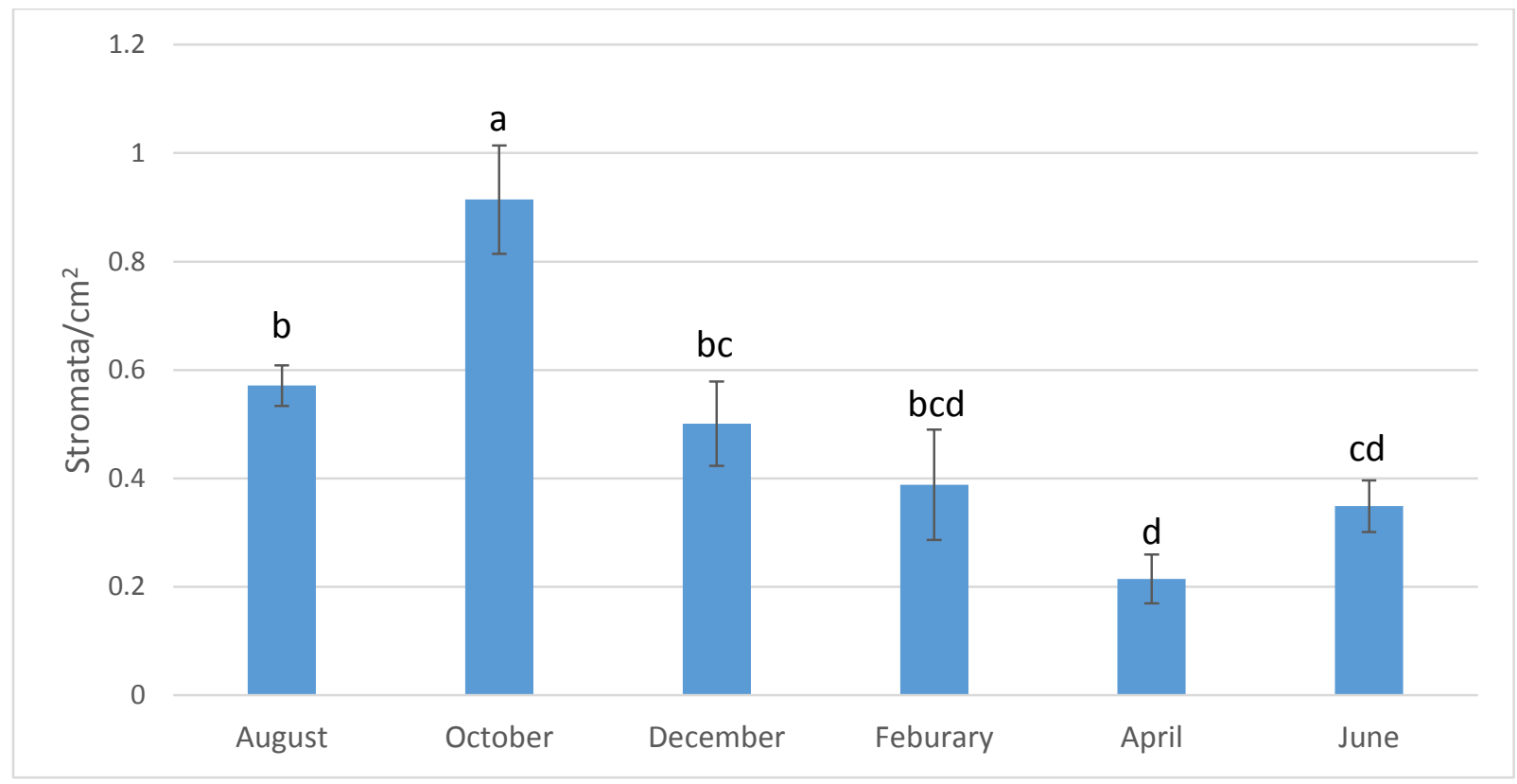

Figure 2.12: Average stromata counts $\left(\right.$ stromata $/ \mathrm{cm}^{2}$ ) for all hosts by measurement period; error bars represent +/- 1 SE 
Effects of temperature and precipitation on sporulation and growth

Temperature and precipitation measurements for the 2015-2016 period are

summarized in Table 4. Increase in temperature was positively correlated with the increase in stromata density at $r=0.819(P<0.05)$ and canker expansion at $r=0.922(P<0.01)$. Increased precipitation was positively correlated with stromata density at $r=0.741$, but this relationship was not significant.

Table 2.4: Average precipitation and temperature values for each measurement period

\begin{tabular}{|l|r|r|r|r|r|r|}
\hline & $\begin{array}{l}\text { June- } \\
\text { August }\end{array}$ & $\begin{array}{l}\text { August- } \\
\text { October }\end{array}$ & $\begin{array}{l}\text { October- } \\
\text { December }\end{array}$ & $\begin{array}{l}\text { December- } \\
\text { February }\end{array}$ & $\begin{array}{l}\text { February - } \\
\text { April }\end{array}$ & \multicolumn{2}{l|}{$\begin{array}{l}\text { April - } \\
\text { June }\end{array}$} \\
\hline Precipitation $(\mathrm{mm})$ & 122.3 & 73.7 & 58.8 & 81.7 & 85.4 & 107.1 \\
\hline Temp (max) $\left({ }^{\circ} \mathrm{C}\right)$ & 27.7 & 27.7 & 16.8 & 8.0 & 10.9 & 19.8 \\
\hline Temp (avg) $\left({ }^{\circ} \mathrm{C}\right)$ & 22.6 & 21.7 & 11.2 & 3.0 & 5.5 & 13.8 \\
\hline Temp (min) $\left({ }^{\circ} \mathrm{C}\right)$ & 17.6 & 15.7 & 5.6 & -2.0 & 0.1 & 7.9 \\
\hline
\end{tabular}

\section{Visual canker ranking}

Cankers resulting from inoculations with the Weekly isolate were visually ranked on a scale from 1-5 based on the characteristic described in Table 2.3 with the most resistant rank designated as a 1 and least resistant as a 5. Cankers on American chestnuts had highest ordinal ranking with an average of 3.6 (Figure 2.13). Chinese chestnuts had the lowest average canker rank with an average of 1.95 and were statistically lower than all other backgrounds except B2F2. TACF hybrid generations B2F3, B3F2, and European chestnuts had a higher average rank but were not statistically different from American. B2F2 chestnuts were statistically different from all hosts but European, B2F3, and Chinese chestnuts and had an average of 2.5. 


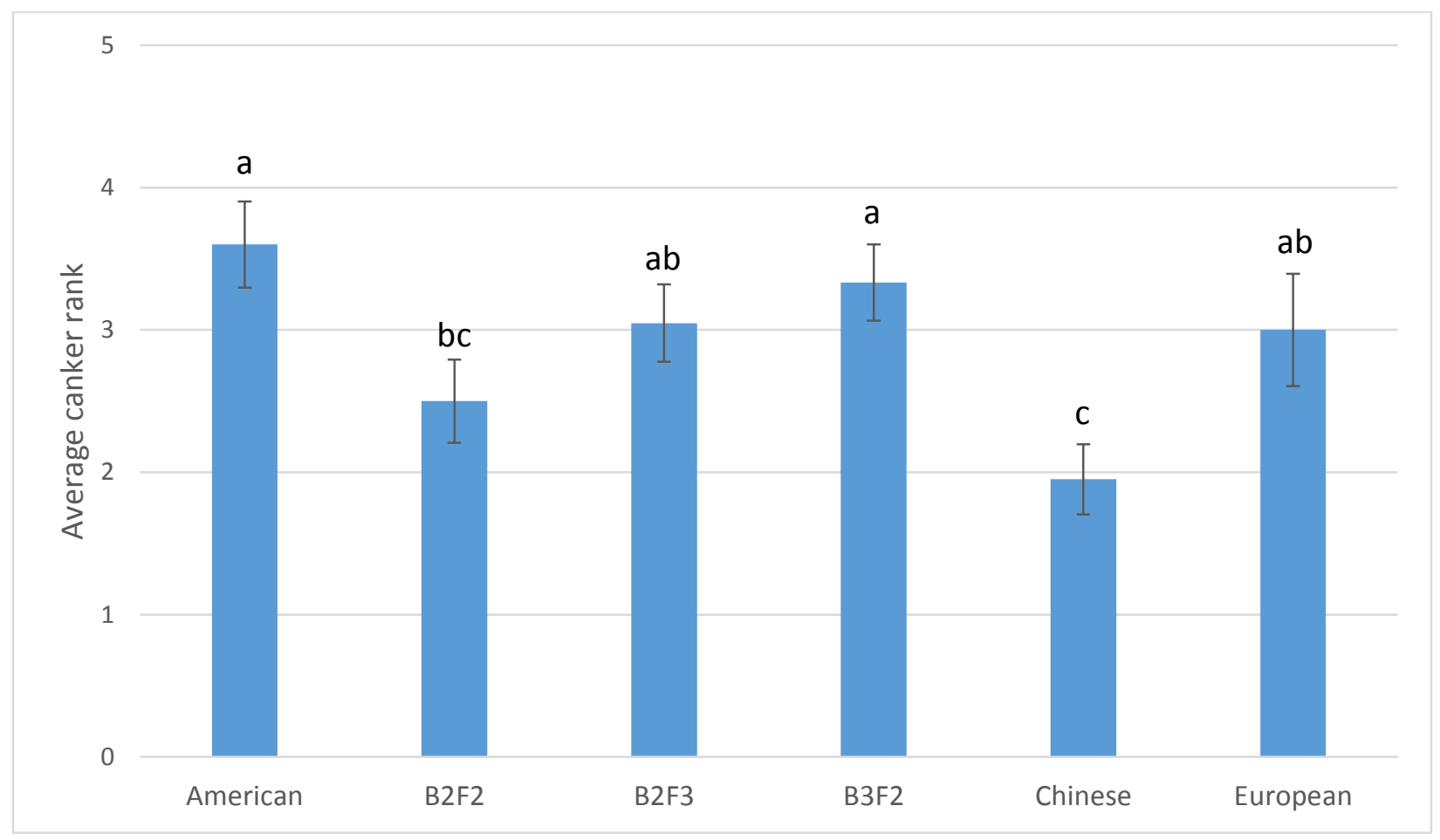

Figure 2.13: Average virulent canker rank for each host background; error bars represent +/- 1 SE

$\underline{\text { Stem diameter change and callus estimates }}$

The average change in stem diameter for $\mathrm{DBH}$ and the diameters at each inoculation site for all host backgrounds revealed a significant higher average increase in diameter for the Chinese background at $1.39 \mathrm{~cm}$ over the duration of the study, but these values were not significantly different than B2F2 chestnuts (Figure 2.14). American chestnut had the smallest average increase in diameter of $0.72 \mathrm{~cm}$. B2F3, B3F2, and European had average diameter increases between American and Chinese chestnuts, but these were not significantly different than that of American chestnut. 


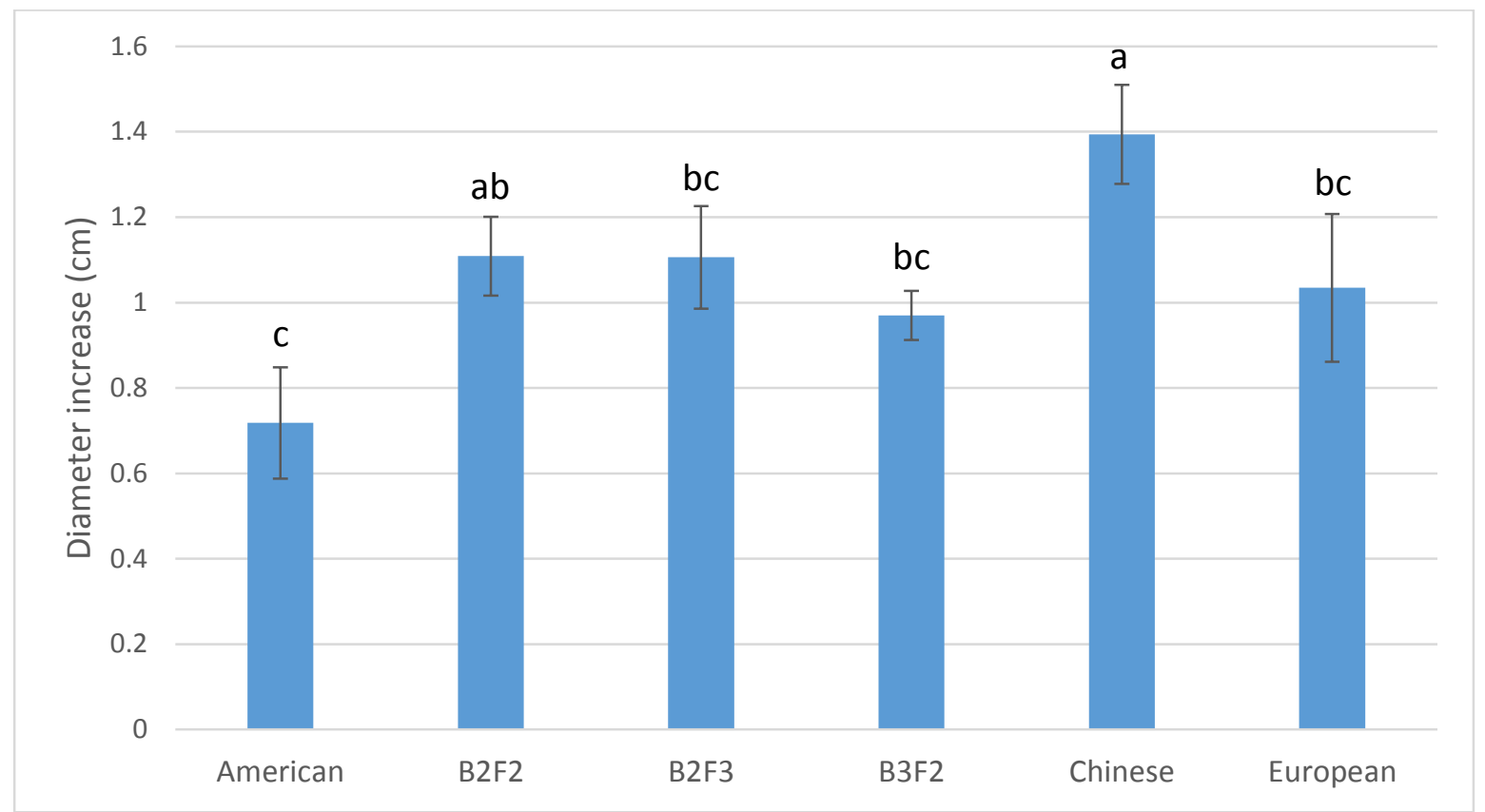

Figure 2.14: Diameter increase from start to end of study across all host backgrounds; error

$$
\text { bars }=+/-1 \mathrm{SE}
$$

At the end of the study, callus growth was calculated by subtracting the change in diameter of the stem at virulent Weekly inoculation sites from the average change in diameter for all measurement sites on the tree. Callus growth calculations for virulent canker sites revealed Chinese had an average increase of $0.24 \mathrm{~cm}$ above the average diameter increase at all other measurement points (Figure 2.15). American chestnuts had an average decrease in diameter of $-0.2 \mathrm{~cm}$ at virulent canker sites, and this value was similar to B3F2 and European chestnuts. Chinese, B2F2, and B2F3 chestnuts all had significantly larger average increase in diameter at virulent canker sites than American. 


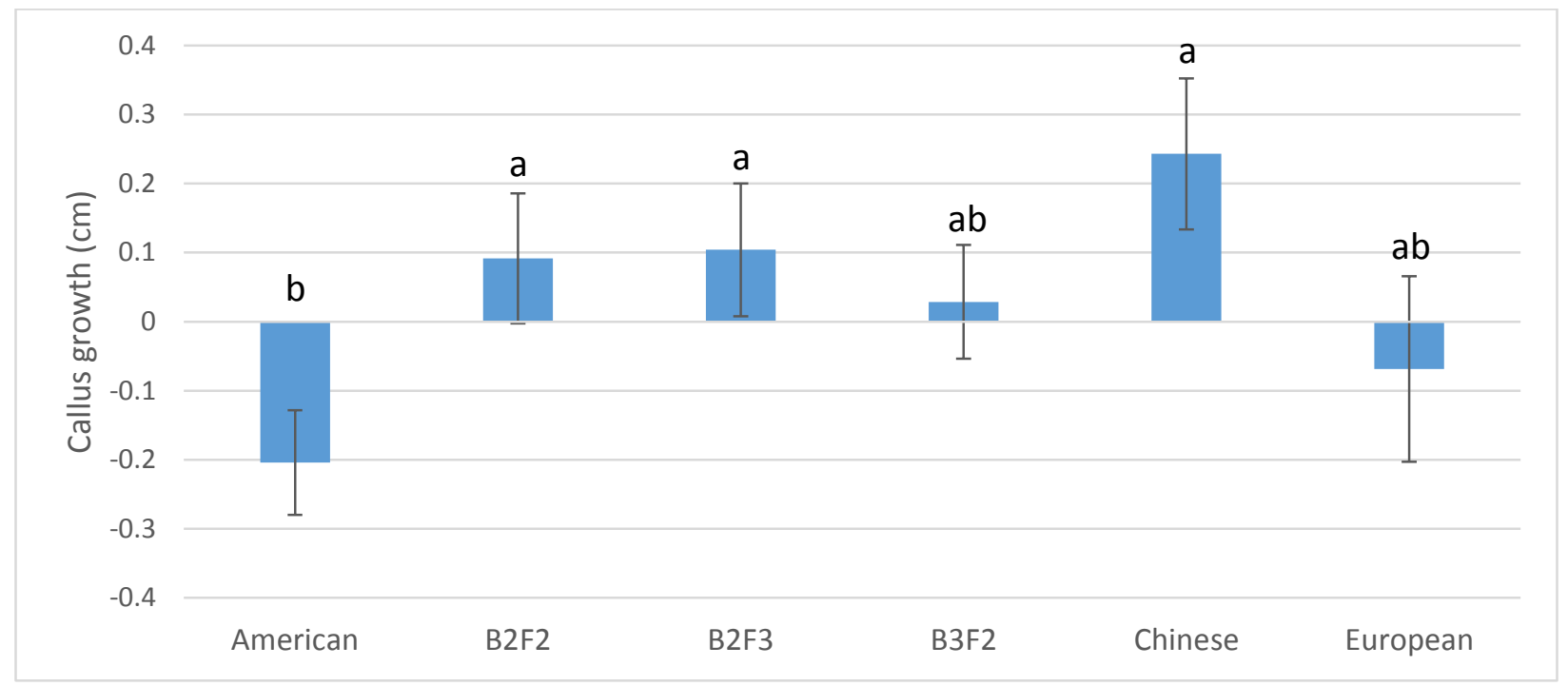

Figure 2.15: Callus growth estimate from diameter increase for virulent Weekly canker inoculation sites; error bars represent +/- $1 \mathrm{SE}$

\section{Percent mortality}

The highest mortality occurred for American chestnuts (35\% of trees) (Table 2.5). TACF hybrid generations B2F3, B3F2, and European had the followed with mortality of $19 \%, 17 \%$, and $10 \%$, respectively. TACF hybrid generation B2F2 only had $5 \%$ mortality, and all Chinese chestnuts survived until the end of the study.

Table 2.5: Percent mortality at end of study by host background; Significance values represent pairwise comparison results from Kaplan-Meier survival analysis $(\alpha=0.003)$

\begin{tabular}{|l|c|}
\hline \multicolumn{1}{|c|}{ Species } & $\begin{array}{c}\text { Percent } \\
\text { Mortality }\end{array}$ \\
\hline American (A) & $35 \%$ \\
\hline B2F2 (AB) & $5 \%$ \\
\hline B2F3 (AB) & $19 \%$ \\
\hline B3F2 (AB) & $17 \%$ \\
\hline Chinese (B) & $0 \%$ \\
\hline European (AB) & $10 \%$ \\
\hline
\end{tabular}


$\underline{\text { Reisolation from study cankers }}$

All reisolations from virulent cankers resulted in the recovery of isolates with Weekly's genotype (Table 2.6). For hypovirulent cankers, the Weekly-CHV1 genotype was recovered from eight of nine cankers. Isolations from two Weekly-CHV1 (SGHV) cankers which continued to grow throughout the study resulted in two virulent isolates with non-Weekly-CHV1 genotypes. For the subset of isolates included in the dsRNA extraction, the CHV1 hypovirus was recovered for the Weekly-CHV1 isolates but not from the Weekly virulent nor from a virulent isolate recovered from growing Weekly-CHV1 cankers (SGHV) (Table 2.6; Figure 2.16).

Table 2.6: Isolate genotype and dsRNA confirmation for reisolated C. parasitica isolates from selected cankers

\begin{tabular}{|c|c|c|c|c|c|}
\hline \multirow{11}{*}{ 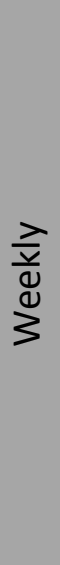 } & Species & Plot & Position & Genotype & dsRNA \\
\hline & American & 1 & 8 & $2211-11$ & \\
\hline & American & 4 & 54 & 2211-11 & - \\
\hline & $\mathrm{B} 2 \mathrm{~F} 2$ & 1 & 123 & 2211-11 & \\
\hline & B2F3 & 4 & 9 & 2211-11 & \\
\hline & B3F2 & 1 & 125 & 2211-11 & \\
\hline & B3F2 & 1 & 38 & 2211-11 & \\
\hline & Chinese & 4 & 5 & 2211-11 & \\
\hline & Chinese & 4 & 78 & 2211-11 & \\
\hline & European & 5 & 93 & 2211-11 & \\
\hline & European & 1 & 34 & 2211-11 & \\
\hline \multirow{11}{*}{ 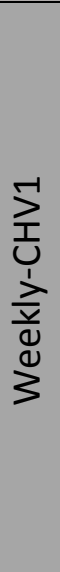 } & American & 4 & 10 & 2211-11 & + \\
\hline & American (SGHV) & 1 & 8 & 2121-21 & \\
\hline & American (SGHV) & 1 & 53 & $2112-11$ & - \\
\hline & B2F2 & 1 & 123 & 2211-11 & + \\
\hline & $\mathrm{B} 2 \mathrm{~F} 2$ & 5 & 9 & $1221-11$ & \\
\hline & $\mathrm{B} 2 \mathrm{~F} 3$ & 1 & 35 & 2221-11 & + \\
\hline & $\mathrm{B} 2 \mathrm{~F} 3$ & 5 & 80 & 2211-11 & \\
\hline & B3F2 & 4 & 1 & 2211-11 & + \\
\hline & B3F2 & 5 & 101 & 2211-11 & \\
\hline & European & 5 & 93 & 2211-11 & \\
\hline & European & 1 & 34 & 2211-11 & + \\
\hline
\end{tabular}




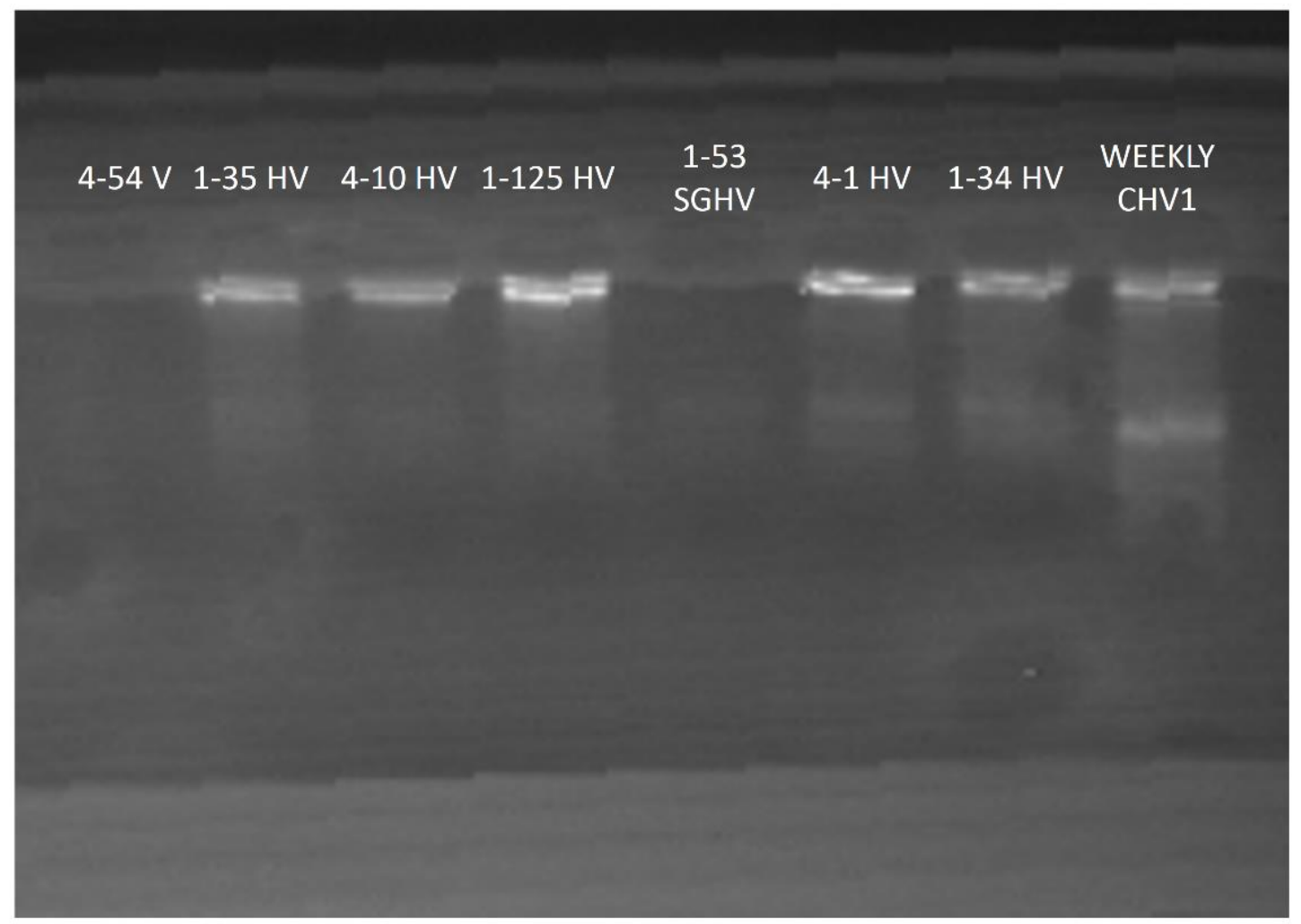

Figure 2.16: dsRNA extraction gel photo for selected reisolated hypovirulent isolates with the original Weekly CHV1 as a positive control; sample names (i.e. 4-54 V) represent plot (4), position within plot (54) and treatment (V; Weekly) 


\section{DISCUSSION}

Many factors contribute to the response of a chestnut host once it is infected with the chestnut blight fungus. Among them, host resistance and pathogen virulence are considered most influential. Given the overwhelming presence and high vic genotype diversity of $C$. parasitica across North America, the use of hypoviruses as biocontrols is limited. Therefore, breeding for higher levels of host resistance serves as one of the most practical solutions for the restoration of the American chestnut.

The American Chestnut Foundation program has produced many generations of American $x$ Chinese hybrids of varying levels of resistance. The current TACF screening program measures resistance by direct stem inoculation and uses early symptoms of susceptibility to cull individuals of low resistance. The long-term host response and ultimately, survival is assumed from these early screening results.

In this research, a number of measurements and analyses were used to compare three individual generations of TACF hybrids from various lineages to populations of American, Chinese, and European chestnut. The results were intended to capture components that contribute to host resistance as well as the pathogenicity of virulent and hypovirulent $C$. parasitica strains. Given the limited growth of Weekly-CHV1, the following discussion largely will be about the observations made for inoculations made with virulent Weekly strain.

\section{Assessing resistance through linear growth analyses}

Linear growth is commonly used to assess the pathogen's ability to colonize the host tissue in the presence of host resistance. A large part of this resistance is expressed as the host 
attempts to produce callus tissue associated with impeded growth of the fungus. All hosts are capable of producing callus tissue as it is a response to wounding. When chestnut is infected with $C$. parasitica, the host must produce this tissue faster than the pathogen is able to invade the host in order to survive the infection. In the case of American chestnut, the host often is unable to produce callus tissue rapidly enough, and therefore, the chestnut blight fungus grows largely unimpeded by the host's attempt to compartmentalize it (Hebard, 1984). Therefore, linear growth is a direct measure of the ability of the host to slow the growth of the fungus or in other words, the inability of the fungus to invade the host tissue due to the host's active resistance during the growing season.

The analysis of the final linear growth measurements found all hosts to have similar measurements except for cankers on Chinese chestnut which had significantly less growth than all hosts aside from American (Figure 2.4). For trees in this study, the death of a stem rendered the cankers unusable as the pathogen began to grow unimpeded through the dead tissue of the tree. This could lead to misrepresentative results. The high rate of mortality for American chestnuts (35\%; Table 2.5 ) likely resulted in such a misrepresentation as the most susceptible individuals were not included in the final linear growth analysis. Mortality was observed across all host backgrounds except Chinese. Therefore, more average linear growth for all other host backgrounds may have been seen had it been possible to include the cankers on dead trees in the analysis of the final linear growth measurements (Table 2.5). To address this issue, a repeated measures ANOVA for linear growth was performed to include every measurement taken prior to tree death (Figure 2.7). Here, B2F2 and European chestnuts' average linear growth was significantly similar to both American and Chinese chestnuts. This is indicative of a 
greater level of resistance among the B2F2 and European chestnuts in comparison to B2F3, B3F2, and American chestnuts.

In another effort to include data from trees which died during the course of the study, the rate of canker expansion was calculated for the six measurement periods of the study. In this way, the rates of growth seen on hosts which ultimately succumbed to the infection were included in the averaged values until they died. Using these data, no further differences were found among the hosts thus providing a similar conclusion that Chinese chestnut has a higher level of resistance than the other host backgrounds given the smaller canker sizes and lower rates of canker expansion. This finding is not surprising as it has been often documented in previous studies (Graves, 1950; Berry, 1960; Anagsnostakis, 1991).

Overall, final linear growth, repeated measures ANOVA for linear growth, and rate of expansion analyses failed to identify many significant differences among host backgrounds relative to resistance. The TACF hybrid generation B2F2 and European chestnuts were the only backgrounds to demonstrate resistance statistically similar to the Chinese chestnuts. The nature of the TACF program is designed to select individuals that express levels of resistance higher than average while culling others. In this way, overall averages, such as those presented here, can be useful for general comparisons, but the true interest lies in the more resistant individuals within each species or hybrid generation.

When considering the various lineages represented within each species or hybrid generation, lineages which express much higher or lower levels of resistance can be identified (Supplemental Graph 2.2). For example within the B2F2 lineages, the B22 line had the most average linear growth $(25.58 \mathrm{~cm})$ of all included lineages across all host backgrounds while the 
BF2 line had an average of $13.83 \mathrm{~cm}$, which is comparable to the overall average of Chinese chestnut at $13.91 \mathrm{~cm}$. Based on these data, the BF2 line indicates that this lineage achieves a desirable level of resistance while those in the B22 line would likely be culled given their high level of susceptibility. The results of this study, where cankers on American and backcross chestnuts were similar in size while those on Chinese chestnut were smaller, are similar to those previously reported (Hebard et al., 2013).

The measurements and analyses used in this study served as adequate means of assessing host resistance over a longer term study. Equal representation of the descendants from various parentages would have made for a more robust study and allowed for more conclusions to be made regarding individual genotypes among the chestnut species and hybrids included in this study.

Stem diameter growth and callus production

The diameter of Chinese chestnut increased the most over the course of the year while American chestnuts increased the least. While this may be a characteristic of these individual chestnut species, the overall vigor of these hosts also may have been severely decreased for the more susceptible hosts as infections increased in size. As average canker size increases, the average change in stem diameter decreased thus further supporting the hypothesis that the presence of an infection may lower the overall vigor of the host (Figures $2.4 \& 2.14$ ).

The callus production estimates generated in this study were based on the assumption that diameter increase for the whole tree was proportional. Average diameter increases calculated from four measurement points on the tree were used as to control for any instances 
where this assumption did not hold. Callus production is a mechanism by which a host compartmentalizes an infection, and in theory, a resistant host will produce a larger amount of callus tissue than a more susceptible host. Without callus production, the growth of the pathogen results in a sunken lesion without this swollen callus tissue. This explains why there was a negative value associated with the callus production estimate for the highly susceptible American chestnuts while a positive value was found for resistant Chinese chestnuts (Figure 2.15).

Overall, Chinese, B2F2, and B2F3 chestnuts produced a significantly larger average callus than American chestnuts (Figure 2.15). The positive average callus production estimates for these TACF hybrid generations indicate a higher level of resistance than American chestnut. This is supported by the ordinal ranking results, which indicated many more Chinese and B2F2 chestnuts had formed noticeably larger callus compared to American chestnut (Figure 2.12).

\section{Mortality}

As death is the most important result, this study could have continued for a longer period of time to assess host survivorship. Given that cankers continued to expand through the course of a year, canker expansion may not have been controlled by many hosts included in this study. Therefore, survival must be considered a result of the balance of canker expansion and tree growth or an imbalance due to higher rates of tree growth until the infection is effectively compartmentalized.

The highest mortality rates due to a study inoculation were seen for American chestnut (35\%) followed by B2F3 (19\%) and B3F2 (17\%). No Chinese chestnuts succumbed to the 
inoculations, and lower rates of morality were seen for European (10\%) and B2F2 (5\%). The individuals that succumbed to the Weekly infections were smaller diameter trees

(Supplemental Table 2.1). Further, the average DBH of B2F2 trees was slightly higher than average and therefore, a factor that likely contributed to the lower rate of mortality given that canker expansion was not limited by the diameter of the tree. This is further supported by the similar rate of growth observed for Weekly inoculations for B2F2 and American chestnuts.

\section{Stromata production}

Dissemination of $C$. parasitica is considered to be largely dependent on conidia which are formed in pycnidia embedded in stromata. Also, outcrossing, which leads to higher levels of vic diversity, occurs via sexual reproduction inside ascocarps embedded in stromata. Therefore, an estimation of stromata density on a canker is one measure of the pathogen's potential to disseminate both asexual and sexual spores. A host which produces more stromata would therefore be a host which could promote disease progression and higher vic diversity.

In this study, B3F2 chestnuts had the highest average density of 0.65 stromata $/ \mathrm{cm}^{2}$, and Chinese chestnuts had the lowest average of 0.156 stromata $/ \mathrm{cm}^{2}$ (Figure 2.9). These density findings were largely proportional to total stromata counts (Supplemental Figure 2.3). This lower rate of stromata production for Weekly infections on Chinese chestnuts can be considered an effect of its active resistance to the chestnut blight fungus. If the stromata production of $C$. parasitica were reduced by host resistance, the fungus would not have the capability of actively sporulating, which in turn could give rise to additional infections. While these secondary infections also may be resisted, additional infections would have the potential 
to reduce the vigor of the host and potentially lead to tree mortality as these infections girdle the stem. Given the similar rates of stromata production of Chinese and B2F2 chestnuts, a retained level of active resistance may once again be indicated by these data.

\section{$\underline{\text { Visual assessment of cankers }}$}

Large-scale breeding programs, such as that conducted by TACF, depend on estimations of host resistance. These are commonly obtained by visual ranking assessments of canker morphology. This ranking system places a host with no callus formation and heavy sporulation as the most susceptible and a host with callus tissue and no stromata at the lowest. In theory, the rankings should reflect the data which could be obtained through many of the more intensive measurements described here.

The visual rankings for the hosts included in this study directly reflected the linear growth and stromata count data. Therefore, this visual ranking method can be further validated as an adequate assessment for screening purposes. In many ways, callus growth is difficult to measure, and this ranking system may capture this aspect of host resistance more accurately than other methods.

\section{Seasonal effects on stromata production and canker expansion}

The influence of temperature and moisture on the growth of various fungi has been documented in a number of studies. In the case of $C$. parasitica, limited data is available for the activity of the fungus over the course of a year. The climatic conditions of temperature and precipitation were evaluated. 
In this study, temperature was highly correlated with the increase in the growth of $C$. parasitica. This is apparent when considering the change in rates of growth as the seasons progressed over the course of the study. Growth decreased as the autumn and winter months approached, and then growth increased as the temperatures increased with the onset of spring and summer. No cessation of growth was observed for any entire host background throughout the course of the study. The lowest rate of growth of $0.032 \mathrm{~cm}^{2} /$ day was observed on the most resistant Chinese hosts during the months of December-February. Canker orientation in relation to the sun was consider as an explanation for growth during these winter months. In theory, cankers which face a more southwestern aspect would receive more sun and thus experience warmer temperatures than those northeastern facing cankers, but no correlation was observed between growth and aspect.

Both precipitation and temperature were positively correlated with the density of stromata, but only temperature was significant. In this case, as the average temperature increased, the density of stromata increased. Seasonal influences have been previously described in regards to sporulation, namely the autumn months are the peak season for sporulation. Observing the shifts in stromata density during the study, a similar conclusion can be made. Stromata density increased through the autumn months, but as the temperatures decreased during the winter months, stromata density decreased. As temperatures warmed and precipitation increased in the spring, stromata density also increased.

In conclusion, change in temperature and precipitation influenced the growth of $C$. parasitica during this study, and although there was not a significant relationship, precipitation 
seemed to influence stromata production. Among the host backgrounds, shifts in growth and stromata production were largely proportional.

\section{$\underline{\text { Weekly-CHV1 Virulence }}$}

Hypovirulence is a biocontrol phenomenon that has been associated with the control of chestnut blight. In theory, an effective biocontrol would be one that increases host survival through the reduction of the pathogen's virulence and ability to disseminate virulent propagules. Given the intracellular nature of the hypovirus, there must be a unique balance of pathogen virulence and its ability to spread throughout the forest. This is because the virulence of the hypovirulent isolate must be reduced enough for the tree to be able to compartmentalize the infection of the infecting hypovirulent strain to ensure survival, but given the need to naturally disseminate the hypovirus, the fungus must be able to sporulate in the presence of a host's resistance.

The results of this study confirm the severe debilitation of the growth and sporulation of Weekly-CHV1. Interestingly, this isolate was recovered nine months after inoculation indicating that the fungus was still viable despite its inability to invade new tissue for at least a sevenmonth period.

Given that the cankers assessed in this study were initiated with a large plug of inoculum (approx. $1 \mathrm{~cm}$ in diameter), it is unlikely that a germinated, hypovirulent conidium would produce a similar amount of growth in a chestnut wound. Hypovirulent conidia have been shown to be less able to incite infections than virulent conidia (Kenaley, 2010). This severe debilitation of growth creates a situation that requires extremely close proximity to a virulent 
canker for hypovirus transmission to the infecting isolate. For applications of hypovirulent isolates for virulent canker treatment, this minimal growth of the hypovirus donor may not be of much concern as it only needs to grow enough to contact the virulent mycelia, anastomose, and transfer the virus. Despite this, the hypovirulent isolate likely may not disseminate hypovirulent propagules as stromata and subsequent conidia formation may be limited.

The European experience with the natural spread of hypovirulence was likely the result of many contributing factors including lower vic diversity. An additional factor may have been the presence of hypovirulent isolates with a higher level of virulence which allowed them to more readily grow and disseminate hypovirulent conidia to secondary infections. Given the level of genetic diversity of $C$. parasitica documented in eastern North America, hypovirulence as a natural biocontrol may not only be restricted by vegetative incompatibility but also, the level of virulence expressed by $C$. parasitica after hypovirus infection. Here, only a single strain designated "Weekly" was tested. Therefore, these findings can only be assumed for this isolate and different results may have been observed if other isolates had been selected.

\section{SUMMARY}

The variation in resistance to $C$. parasitica measured for the included host backgrounds was too high to assign classes of susceptibility as they have been previously described (Graves, 1950). Instead, intra-species and intra-hybrid groupings based on genetic lineages were the levels at which significant differences in resistance may have been described, but this was not fully explored here due to the high variability in the sample sizes of these groups. Chinese chestnuts were the only chestnuts that were significantly different for many of the 
measurements included here. The repeated measures ANOVA of linear growth, visual canker rankings, and callus estimates distinguished a potential increased resistance for the B2F2 chestnuts.

Precipitation and temperature influenced the overall virulence of Weekly. These data provide insight into the biology of the chestnut blight pathogen during the course of a year and indicate that late summer into early autumn or August through October is the optimal time of the year for both growth and sporulation.

Weekly-CHV1 was highly debilitated in regards to growth and sporulation when inoculated within the woody tissue of all chestnut hosts. Given this finding, questions arise regarding the virulence of other hypovirulent strains and the level of debilitation which occurs with other hypovirus species. Further, the variation of susceptibility of the chestnut species and hybrid generations to hypovirulent strains remains unclear. 


\section{LITERATURE CITED}

Anagnostakis, S. L. 1987. Chestnut blight: the classical problem of an introduced pathogen. Mycologia 79: 23-37.

Anagnostakis, S. L. 1991. Measuring resistance of chestnut trees to chestnut blight. Canadian Journal of Forest Research. 22:568-71.

Berry, F. H. 1960. Relative resistance of some chestnut species and hybrids inoculated with the blight fungus. Plant Disease Reporter. 44(9):716-17.

Biraghi, A. 1953. Possible active resistance to Endothia parastitica in Castanea sativa. Reports of the International Union of Forest Research Organization. 643-45.

Clapper, R. B. 1952. Relative blight resistance of some species and hybrids. Journal of Forestry. 50:453-55.

Cortesi, P. and M. G. Milgroom. 1998. Genetics of vegetative incompatibility in Cryphonectria parasitica. Applied and Environmental Microbiology. 64(8):2988-94.

Enebak, S.A. 1992. Characterization of dsRNA-containing strains of Cryphonectria parasitica recovered from the central Appalachian. PhD Thesis, West Virginia University, Morgantown.

Evans, A. M. and A. J. Finkral. 2010. A new look at spread rates of exotic diseases in North American forests. Forest Science. 56(5):453-59.

Graves, A. H. 1950. Relative blight resistance in species and hybrids of Castanea. Phytopathology. 40(12): 1125-31.

Hebard, F. 2005. The backcross breeding program of the American Chestnut Foundation. Journal of the American Chestnut Foundation. 19(2):55-78. 
Hebard, F.V., Georgi, L., Donahue, J., Bevins, D., and Coalson, E. 2013. Meadowview Notes 2011-2012. Journal of the American Chestnut Foundation 27(1):19-25.

Heiniger, U. and D. Rigling. 1994. Biological control of chestnut blight in Europe. Annual Review Phytopathology. 32:581-99.

Hillman, B. I., B. T. Halpern, and M. P. Brown. 1994. A viral dsRNA element of the chestnut blight fungus with a distinct genetic organization. Virology. 201(2):241-50.

Hillman, B. I. and N. Suzuki. 2004. Viruses of the chestnut blight fungus, Cryphonectria parasitica. Advances in Virus Research. 63:423-72.

Jaynes, R. A., \& Elliston, J. E. 1978. Control of Endothia parasitica cankers on American chestnut sprouts with hypovirulent strains. In Proceedings of the American Chestnut Symposium. W.L. MacDonald, F.C. Cech, J. Luchok, and C. Smith, eds. WV Univ. Books, Morgantown. 110-114.

JMP ${ }^{\circledR}$, Version 10. SAS Institute Inc., Cary, NC, 1989-2007.

Kenaley, S. C. 2010. The evaluation of virulent and hypovirulent inocula of the chestnut blight fungus, Cryphonectria parasitica, to incite infection and spermatize virulent cankers. Ph.D. dissertation. Davis College of Agriculture, Natural Resources, and Design. West Virginia University.

MacDonald, W. L. and D. W. Fulbright. 1991. Biological control of chestnut blight: use and limitations of transmissible hypovirulence. Plant Disease. 75(7):656-61.

Merkel, H. W. 1905. A deadly fungus on the American chestnut. New York Zoological Society, 10th Annual Report, pp. 97-103.

Milgroom, M. G. and P. Cortesi. 2004. Biological control of chestnut blight with 
hypovirulence. A critical analysis. Annual Review of Phytopathology. 42:311-38.

Morris, J. T. and J. A. Dodds. 1979. Isolation and analysis of double-stranded RNA from virusinfected plant and fungal tissue. Phytopathology. 69(8):854-58.

Newhouse, A. E., L. D. Polin-McGuigan, K. A. Baier, K. E. R. Valletta, W. H. Rottmann, T. J. Tschaplinski, C. A. Maynard, and W. A. Powell. 2014. Transgenic American chestnuts show enhanced blight resistance and transmit the trait to T1 progeny. Plant Science. 228. 88-97.

Nuss, D. L. 2005. Hypovirulence: Mycoviruses at the fungal-plant interface. Nature 3:63242.

Pavari, A. 1949. Chestnut blight in Europe. Unasylva 3:8-13.

Peever, T. L., Y. Liu, and M. G. Milgroom. 1997. Diversity of hypoviruses and other double-stranded RNAs in Cryphonectria parasitica in North America. Phytopathology. 87(10): 1026-33.

PRISM Climate Group, Oregon State University, http://prism.oregonstate.edu, created 4 Feb 2004.

Schlarbaum, S., S. L. Anagnostakis, and M. C. Morton. (1992) Evaluation of experimental chestnut plantings in Eastern North America. pages 52-56 in: Proceedings of the International Chestnut Conference, M. L. Double and W. L. MacDonald, eds., West Virginia University.

Short, D. P. G., M. Double, D. L. Nuss, C. M. Stauder, W. L. MacDonald, and M. T. Kasson. 
2015. Multilocus PCR Assays Elucidate Vegetative Incompatibility Gene Profiles of Cryphonectria parasitica in the United States. Applied and Environmental Microbiology. 81(17): 5736-42.

Smart, C. D., W. Yuan, R. Foglia, D. L. Nuss, D. W. Fulbright, and B. I. Hillman. 1999. Cryphonectria hypovirus 3, a virus species in the family Hypoviridae with a single open reading frame. Virology. $\mathbf{2 6 5 ( 1 ) : 6 6 - 7 3 .}$

\section{Zhang, B., A. D. Oakes, A. E. Newhouse, K. M. Baier, C. A. Maynard, and W. A. Powell.} 2013. A threshold level of oxalate oxidase transgene expression reduces Cryphonectria parasitica-induced necrosis in a transgenic American chestnut (Castanea dentata) leaf bioassay. Transgenic Research. 22(5): 973-82.

\section{SUPPLEMENTAL TABLES}

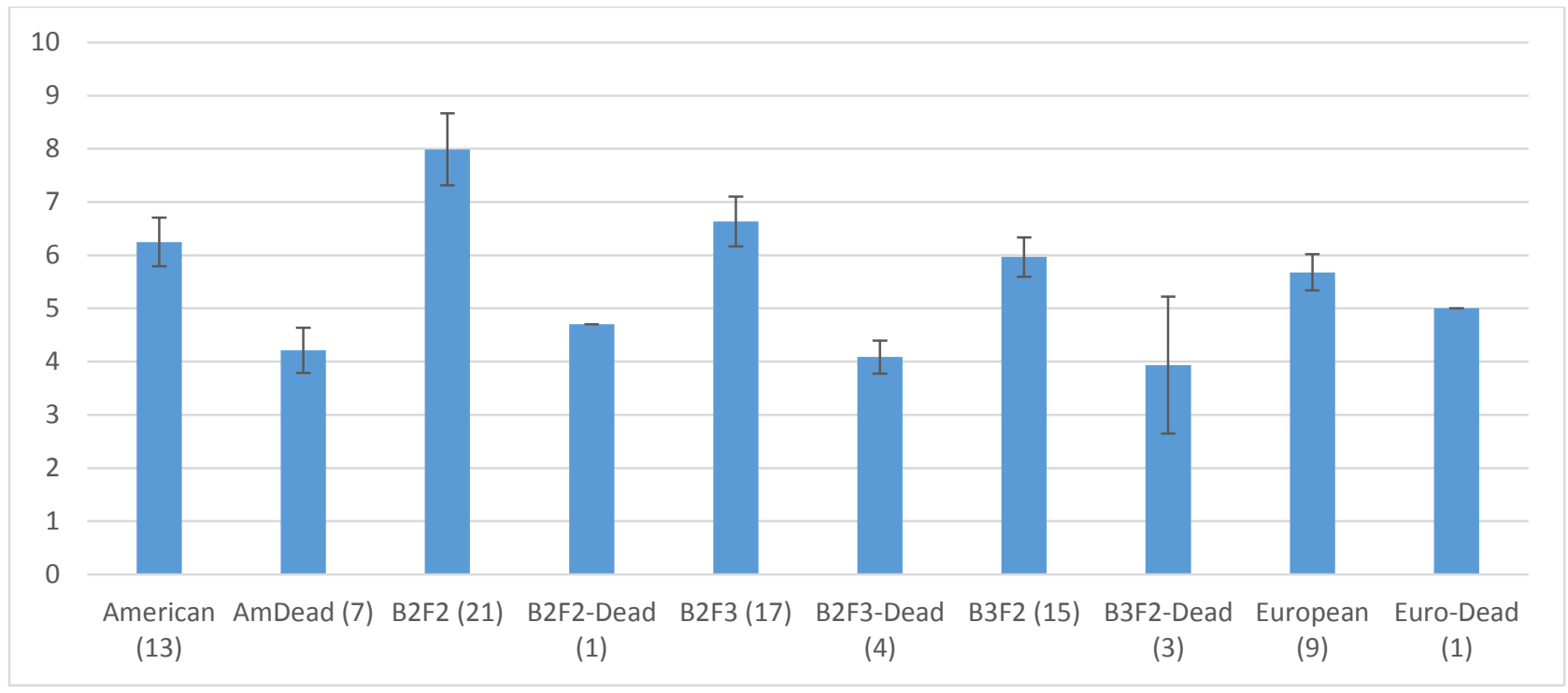

Supplemental Figure 2.1: Comparison of average diameters of living and dead hosts; error bars represent +/- 1 SE 


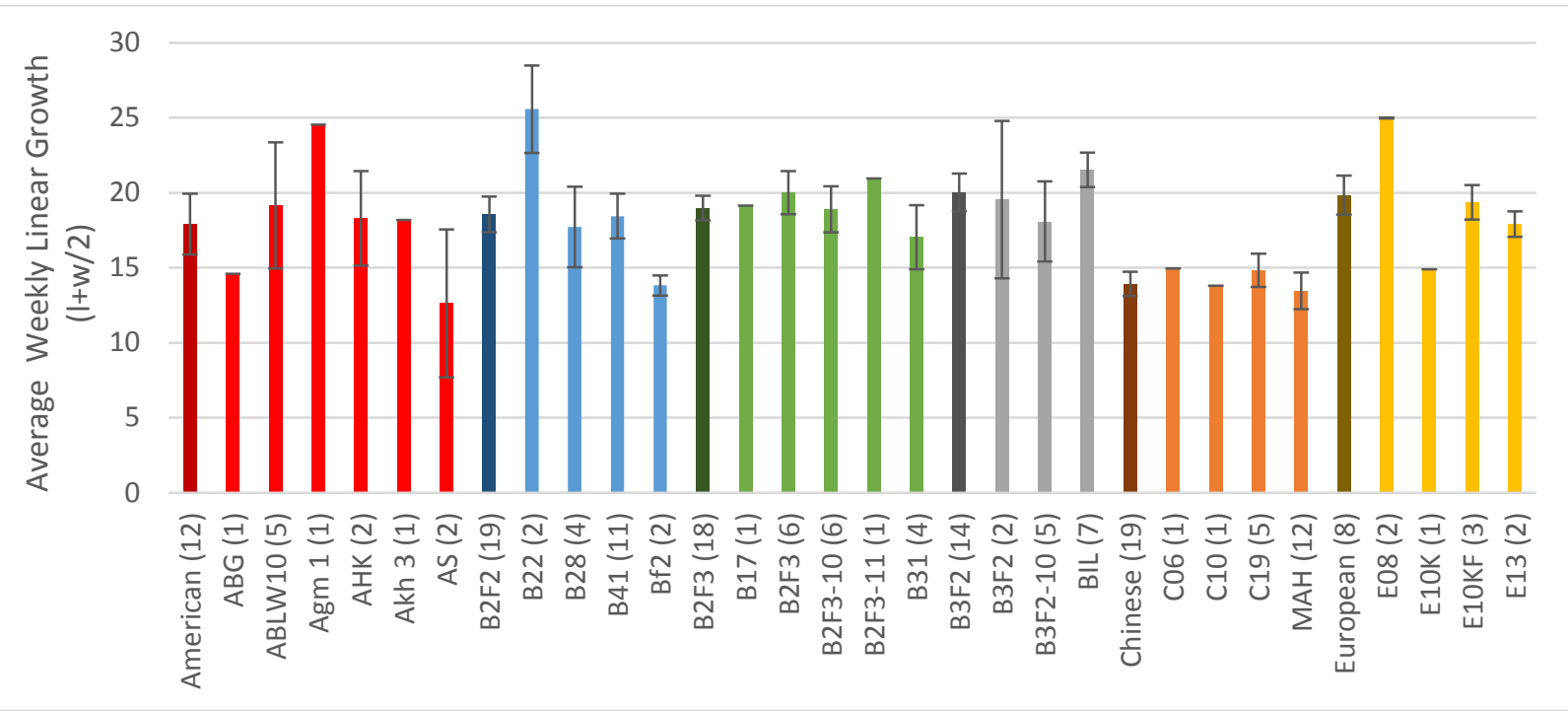

Supplemental Figure 2.2: Average linear growth 12-months post-inoculation by host background and lineage; error bars represent +/- 1 SE

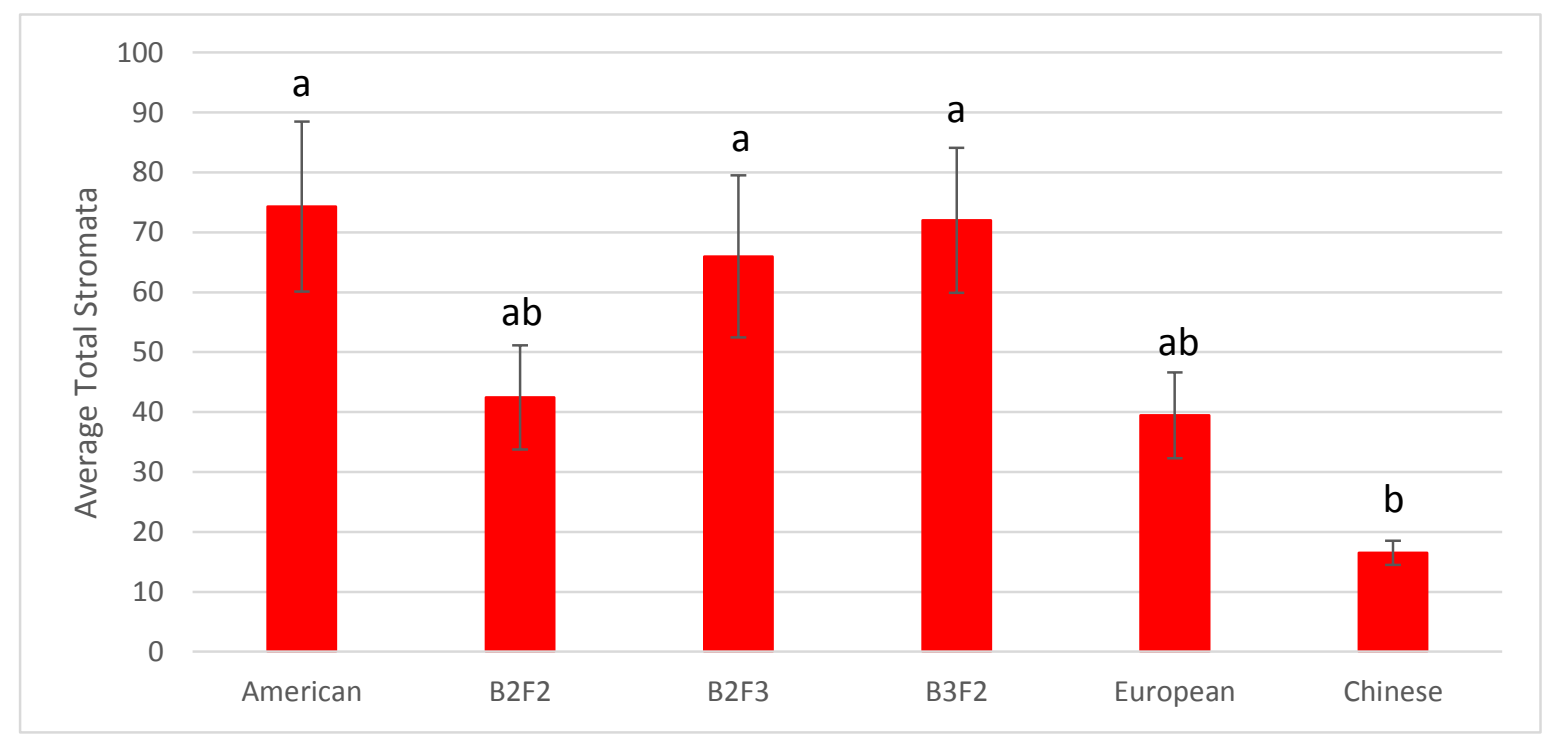

Supplemental Figure 2.3: Average total stromata counts across all host backgrounds; error bars represent +/- 1 SE 
CHAPTER 3: EMPLOYING AN EXCISED-LEAF ASSAY TO ASSESS CHESTNUT HOST RESISTANCE AND CRYPHONECTRIA PARASITICA VIRULENCE

\section{ABSTRACT}

Many efforts have been initiated to restore the American chestnut (Castanea dentata) to its former prominence after its devastation by the chestnut blight pathogen Cryphonectria parasitica in North America. The approaches have included crossbreeding American chestnuts with resistant Asiatic chestnuts ( $C$. mollissima and $C$. crenata), incorporating an oxalic oxidase gene from wheat into the American chestnut genome, and the deployment of hypoviruses to attenuate the virulence of the chestnut blight pathogen. For each of these approaches, investigators routinely measure the growth of artificial infections of $C$. parasitica in chestnut stems for resistance screening. This process takes between 2 and 5 years for trees are from seed or tissue culture, depending on the cross type being tested. Therefore, any effort to reduce this time to identify resistance is valuable for these research efforts. An excised-leaf assay that utilizes newly produced leaves has been shown to produce results in less than seven days, eliminating the need for larger woody stems (Newhouse et. al, 2014). This comparative method uses the measurement of necrotic lesions that arise from artificial inoculations of $C$. parasitica on the leaf midvein. The objective of this study was to test the suitability of the leaf assay to determine intermediate levels of host resistance and pathogen virulence for a collection of chestnut species and American $x$ Chinese hybrids using a virulent strain (Weekly) and an isogenic, hypovirulent (Weekly-CHV1 (Euro7)) strain. The virulent Weekly strain (39.9\%) infected at half of the rate of hypovirulent Weekly-CHV1 (79.4\%). Overall average lesion area was significantly larger for Weekly-CHV1 infections $\left(86.4 \mathrm{~mm}^{2}\right)$ than virulent Weekly $(58.1$ 
$\mathrm{mm}^{2}$ ). Leaf-lesion areas averaged for individual host backgrounds were similar to the respective levels of host resistance described in previous studies, but no correlation was found between leaf-lesion area and canker linear growth data collected in a parallel study for each respective tree (Chapter 2). These findings pose questions regarding the sensitivity, precision, and accuracy of the leaf assay method. Further, the level of virulence of hypovirulent Weekly-CHV1 poses questions regarding the fitness of hypovirus-infected strains when grown on non-woody chestnut substrates.

\section{INTRODUCTION}

Chestnut blight, caused by the fungal pathogen Cryphonectria parasitica, has been an ecologically devastating canker disease of members of the genus Castanea, particularly $C$. dentata and C. ozarkensis. Varying levels of susceptibility to the chestnut blight pathogen have been described among chestnut species with Chinese chestnut ( $C$. mollissima) as most resistant and American chestnut (C. dentata) as the least (Graves, 1950; Anagnostakis, 1991; Berry, 1960; Clapper, 1952). A number of restoration approaches for American chestnut were initiated following the introduction of $C$. parasitica and its subsequent devastation of native chestnut populations in eastern North American (Merkel, 1905). Organizations, such as The American Chestnut Foundation (TACF), were established in an effort to produce resistant American $\mathrm{x}$ Chinese chestnut hybrids through crossbreeding (Hebard, 2005). After the discovery of hypovirulence, there were many efforts focused on the deployment of hypoviruses as a natural biocontrol agents of chestnut blight (Biraghi, 1953; MacDonald and Fulbright, 1991). While this phenomenon has been associated with various levels of control of $C$. parasitica in Europe, the 
North American experience has seen limited success due to a number of restricting factors (Milgroom and Cortesi, 1999, Short et al. 2015). Along with advances in molecular techniques, a recent development using transgenic technology has generated American chestnut trees that express levels of resistance comparable to Chinese chestnut through the incorporation of an oxalic oxidase gene from wheat (Zhang et al., 2013; Newhouse et al., 2014).

Despite differences in fundamental approaches, each of these research efforts continues to depend on testing host resistance and/or pathogen virulence relying on methods that utilize large chestnut stems. For resistance breeding programs such as those employed by TACF, testing for resistance is vital to the program. Comparisons of host susceptibility have been performed using a number of inoculation methods on host woody tissue. This requires either the direct inoculation of the stem or the cutting of dormant stems from the tree for inoculation in the laboratory (Jaynes and Elliston, 1978; Elliston, 1978). These methods are limited by the need for the stems large enough to inoculate or sample and the need for incubation periods of five weeks for excised-stem assays and six months for artificial inoculations in the field to permit robust comparisons. Additionally, these programs are subjected to relatively long spans of time from seed planting to resistance screening at approximately two-to-five years of age without any advanced knowledge of susceptibility (Hebard, 1994). Therefore, any effort to shorten that time would be valuable for such programs.

An excised-leaf assay was proposed for resistance screening that provides results within five days and requires only leaves, effectively eliminating the need for the larger stems required for woody stem assays (Newhouse et al., 2014). The objective of this study was to utilize the 
leaf assay procedure to compare the host resistance of American (Castanea dentata), European (C. sativa), Chinese (C. mollisima) and American $\mathrm{x}$ Chinese hybrid chestnuts using a virulent strain (Weekly) and an isogenic hypovirulent strain (Weekly-CHV1(Euro7)) of C. parasitica. The purpose of the study was to validate the leaf assay as a potential resistance screening method for large-scale breeding operations.

\section{MATERIALS AND METHODS}

\section{Experimental design and treatments}

The resistance of American, Chinese, European, and three generations of American $\mathrm{x}$ Chinese hybrids (B2F2, B2F3, B3F2) grown together in a mixed plantation was explored via direct stem-inoculations with virulent (Weekly) and hypovirulent (Weekly-CHV1) (Euro7-CHV1) C. parasitica strains (Chapter 2). The data collected from the field tests were used in the following selection of trees. From the remaining living trees, six trees representing the most susceptible, intermediate, and most resistant trees based on canker linear growth measurements were selected such that two trees represented each susceptibility class for each species or hybrid generation. The European chestnut population was limited to only one intermediate tree due to tree mortality and the presence of smaller leaves that likely resulted from ambrosia beetle infestations.

Seven recently expanded leaves were collected from each selected tree. Leaves large enough to allow two inoculations separated by approximately $40-\mathrm{mm}$ were preferred (i.e. leaves $>15 \mathrm{~cm}$ in length). In the event that a tree had smaller leaves ( $<15 \mathrm{~cm}$ in length) allowing for only one inoculation, a total of 14 leaves were collected. The variation in the phenology of 
bud break required that morphological characteristics, including level of expansion and leaf color, were used to estimate the ideal level of leaf development; fully-expanded leaves which were no longer tinted red at the tip were selected (Newhouse et. al, 2014). These leaves were typically the third or fourth leaf from the growing branch tip.

\section{Preparation of inoculum}

Isogenic Cryphonectria parasitica isolates Weekly and Weekly-CHV1 were grown on five plates of Difco PDA (39 g/1000 ml) for approximately 5 days at 20.0 C under a 16/8hr light/dark cycle. Prior to leaf inoculations, a \#1 cork borer ( 3-mm diameter) was used to cut inoculum plugs around the growing edge of the colonies or throughout a sterile PDA plate for the negative control.

\section{Collection, inoculation, and incubation procedure}

The following leaf assay procedures are modeled from the procedures developed and described by Newhouse et al. (2014). As leaves were collected, they were placed in labeled Ziploc bags, placed into a cooler, and stored there for transport back to the laboratory. Leaves were labeled in sequence using a Sharpie marker. A 5-mm inoculation site was marked approximately $20-\mathrm{mm}$ from the petiole along the midvein. When a second inoculation site was available, it was placed 40-mm away from the first (Figure 3.1(A)). Leaves were washed in a mild detergent (.005\% Tween-20) and shaken for 1.5 minutes then dried on paper towels.

The 5-mm inoculation sites were then sliced using a \#11 scalpel (Figure 3.1(B)). The cut was approximately halfway through the midvein. A C. parasitica-colonized or sterile control 
medium plug was placed on each inoculation site (Figure 3.1 (C)). For each tree, six leaf inoculations were made for Weekly and Weekly-CHV1 treatments while two inoculations were made for the sterile control. The leaves were then placed in sealable storage trays containing 3 sheets of tri-fold paper towels moistened with $25 \mathrm{ml}$ of water (Figure 3.1(D)). The leaves were placed with the midvein facing upward. The trays were sealed and placed in a dark cabinet and stored for 5 days at room temperature. All inoculations were made within 6 hours of collection.

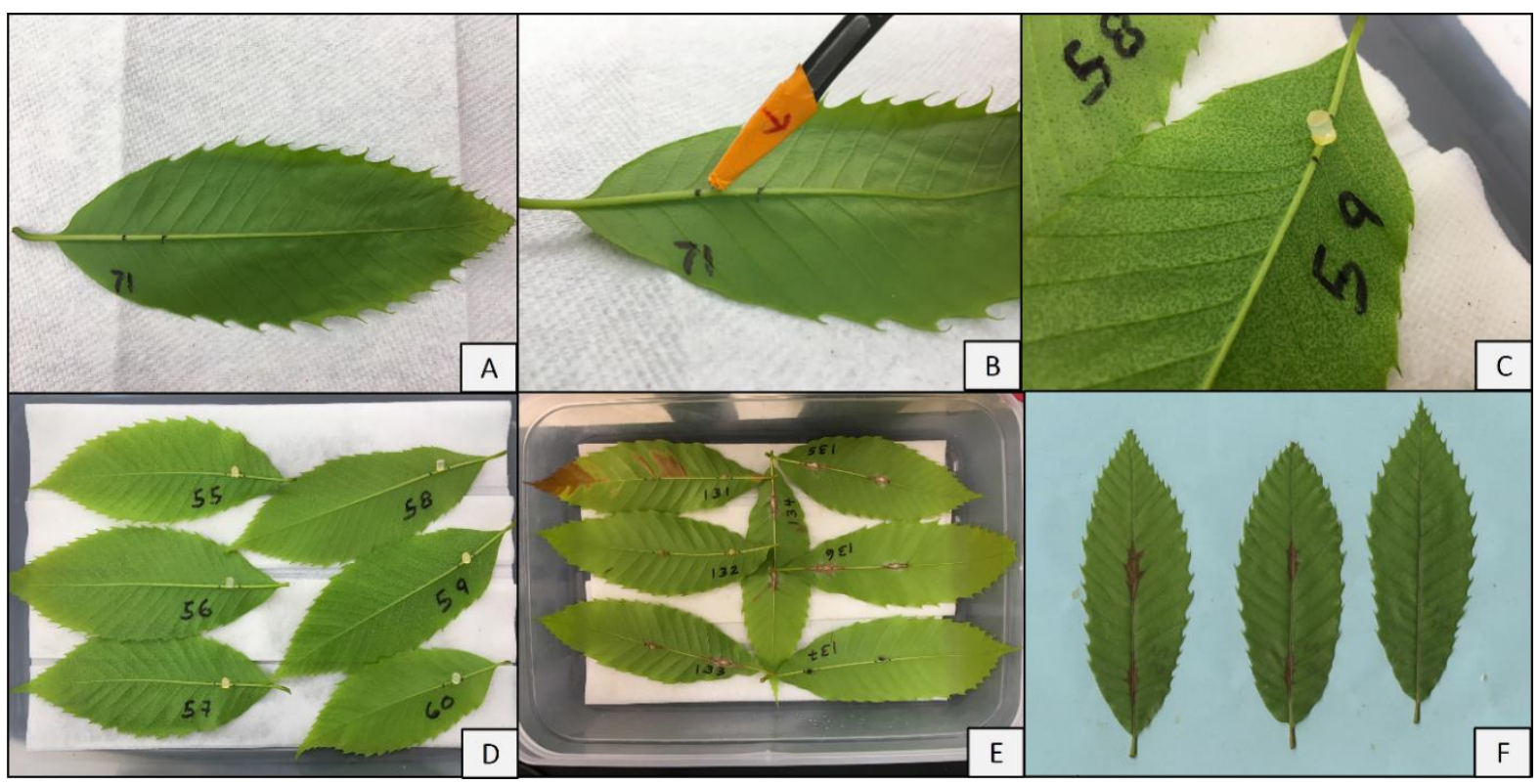

Figure 3.1: Leaf assay procedure as described by Newhouse et. al (2014); A: sequentially labeled leaf with two 5-mm inoculation sites approximately 40-mm apart; B: \#11 scalpel was used to make a cut approximately halfway through the midvein along the 5-mm inoculation site; C: the designated inoculum plug was placed mycelium side down on inoculation site; D: inoculated leaves were placed in a container with moist paper towels; E: leaves in tray after 5 days of incubation; F: necrotic lesions resulting from inoculation with C. parasitica isolates 


\section{Measurements}

Following the 5-day incubation period, leaves were removed from the container, placed on a scanner bed with the midvein up, and scanned into a computer to measure the area of necrosis associated with the inoculation using The American Phytopathological Society: Assess 2.0 software (Lamari, 2002) (Figure $3.1(E, F)$ ).

All necrotic areas were averaged by tree and by treatment. Inoculations which failed to produce a lesion were not included. American chestnut was considered the most susceptible, and therefore was a control by which all other species and hybrid average areas were normalized for comparisons to canker data. By doing so, the measurements were treated and interpreted relative to the control. This approach was necessary so that leaf assay results could be compared to the linear growth data for the selected trees.

\section{$\underline{\text { Reisolation }}$}

For both virulent and hypovirulent inoculations, a small section of leaf (approx. 2-mm x 2-mm) was excised from the necrotic tissue surrounding the inoculation site, bleached in a 1:10 commercial bleach/water solution for 45 seconds, then placed on glucose-yeast extract agar amended with streptomycin and neomycin for reisolation of the causal agent (Appendix A). The white appearance of the Weekly-CHV1 mycelium was used to confirm hypovirulence. For approximately eight failed inoculations per treatment, the original plug was taken from the inoculation site and placed onto PDA as to confirm the viability of the inoculum.

\section{Analyses}

All analyses were performed using JMP ${ }^{\circledR} 10$ software (SAS Institute, Inc., Cary, NC, USA). Mean comparisons served as the main analysis utilized to resolve differences between host 
backgrounds and inoculum sources. To test these factors, a one-way ANOVA with Tukey's HSD for $>3$ dependent variables and Students-t test for $\leq 3$ dependent variables $(\alpha=0.05)$ were selected to make these comparisons as only one independent variable was incorporated into each analysis. A single linear regression was used to test the correlation of normalized lesion and linear growth data.

\section{RESULTS}

The excised-leaf assay procedure was replicated from Newhouse et al. (2014) as described above. Weekly and Weekly-CHV1 produced lesions on leaves from every tree but not on every leaf. Failed inoculations were not included in area analyses. Leaves with visual colonization by organisms other than C. parasitica were removed from the study due to excessive damage to the leaf tissue confounding lesion measurement.

\section{Incidence of infection}

Inoculations were considered successful when a necrotic lesion expanding from the point of inoculation was found (Figure 3.2; A). The absence of such necrosis was scored as an unsuccessful colonization (Figure 3.2; B). The percent of successful infection averaged for all hosts was significantly lower for Weekly inoculations (39.9\%) than hypovirulent Weekly-CHV1 inoculations (79.4\%) (Figure 3.3). The lowest percentage of successful infection (19.4\%) was on B3F2 leaves inoculated with Weekly virulent. Inoculations with Weekly-CHV1 on European chestnuts were successful most often (90.0\%). 


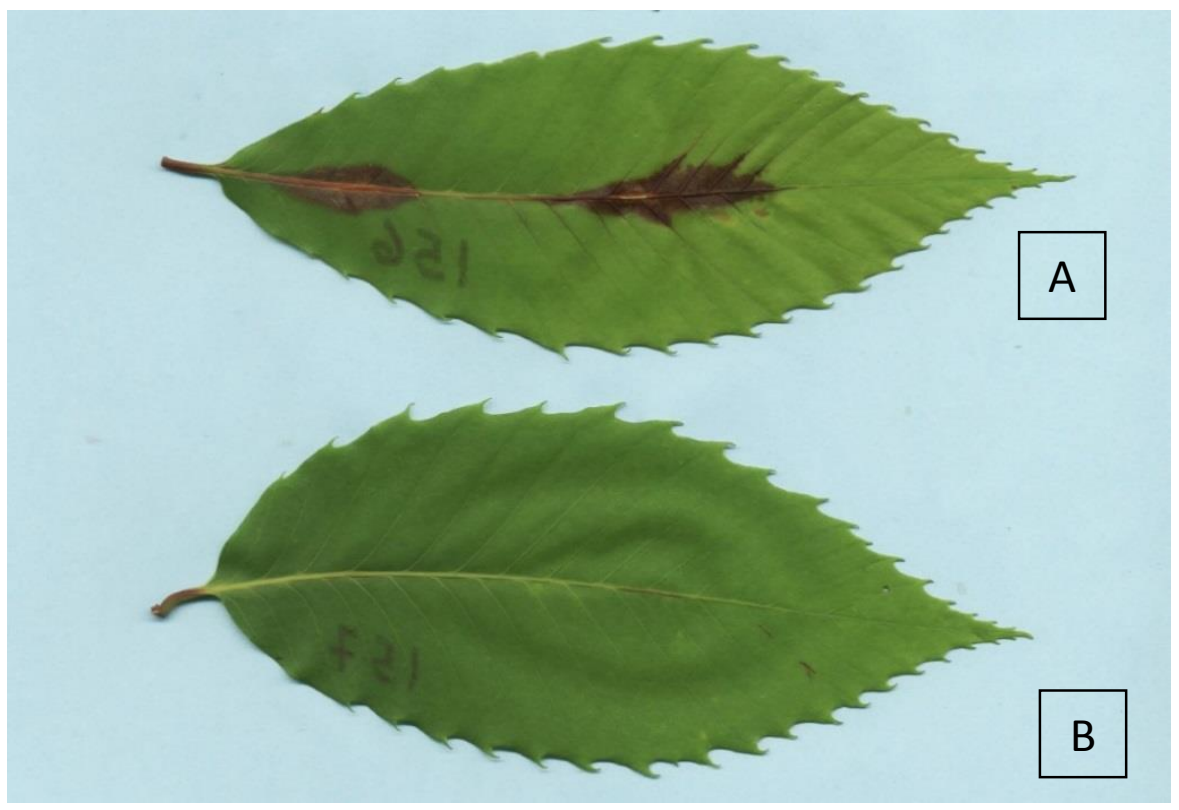

Figure 3.2: Successful (A) and unsuccessful (B) Cryphonectria parasitica infections on chestnut

\section{leaves}

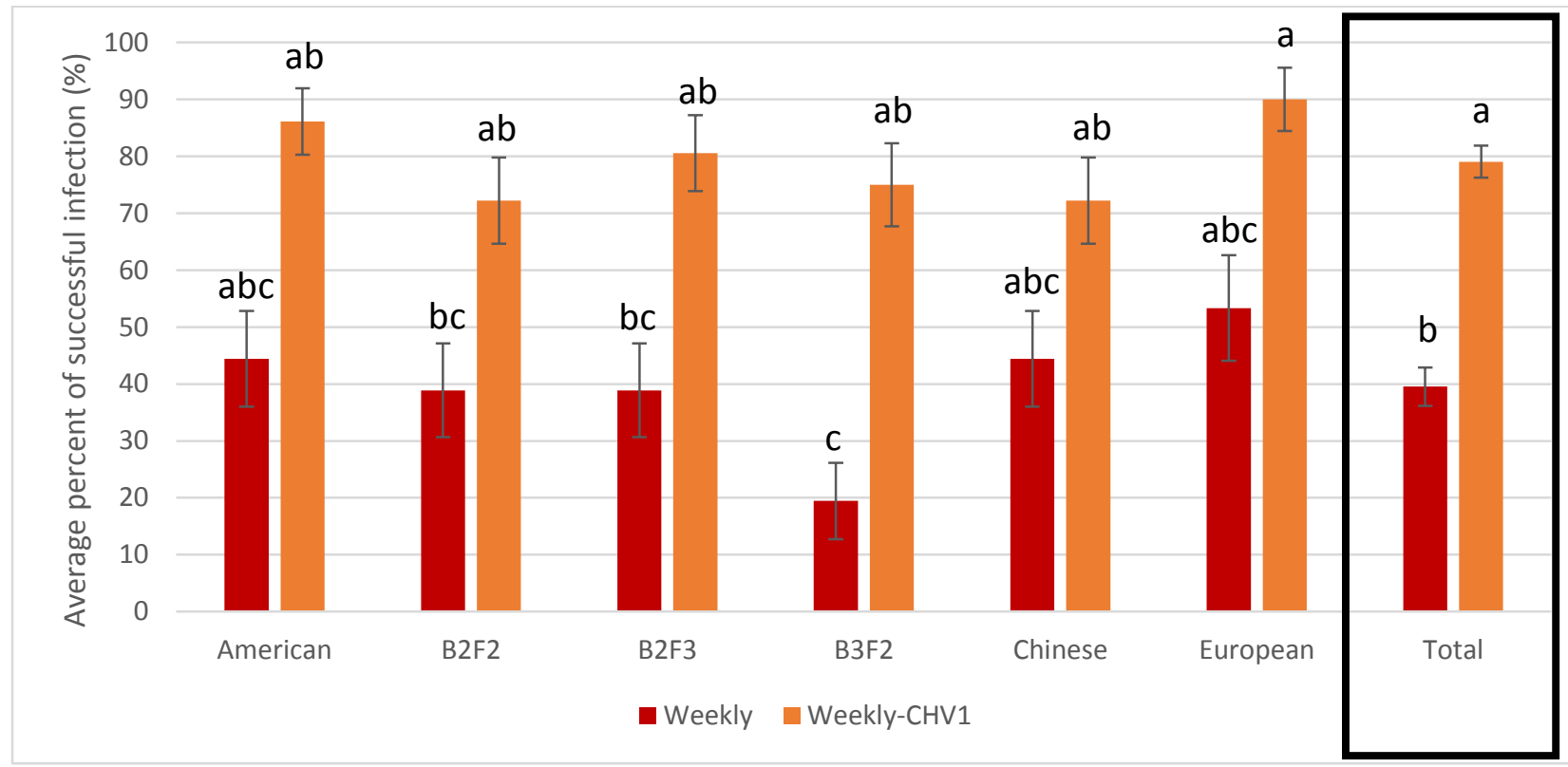

Figure 3.3: Average percent of successful infection for all host backgrounds for Weekly and

Weekly-CHV1; Total percent infection by inoculum type was analyzed separately; Error bars represent +/- 1 SE 


\section{Lesion area by treatment}

Leaf-lesion area averaged for all hosts was significantly smaller for virulent Weekly inoculations $\left(58.1 \mathrm{~mm}^{2}\right.$ ) than Weekly-CHV1 $\left(86.4 \mathrm{~mm}^{2}\right.$ ) (Figure 3.4). There were no lesions formed from control medium only inoculations, and both fungal treatments produced lesions significantly larger than the medium only control. There were no significant differences between inoculum types among the host backgrounds aside from European chestnuts which had an average lesion size of $55.2 \mathrm{~mm}^{2}$ for Weekly inoculations and $125.4 \mathrm{~mm}^{2}$ for WeeklyCHV1 (Figure 3.5).

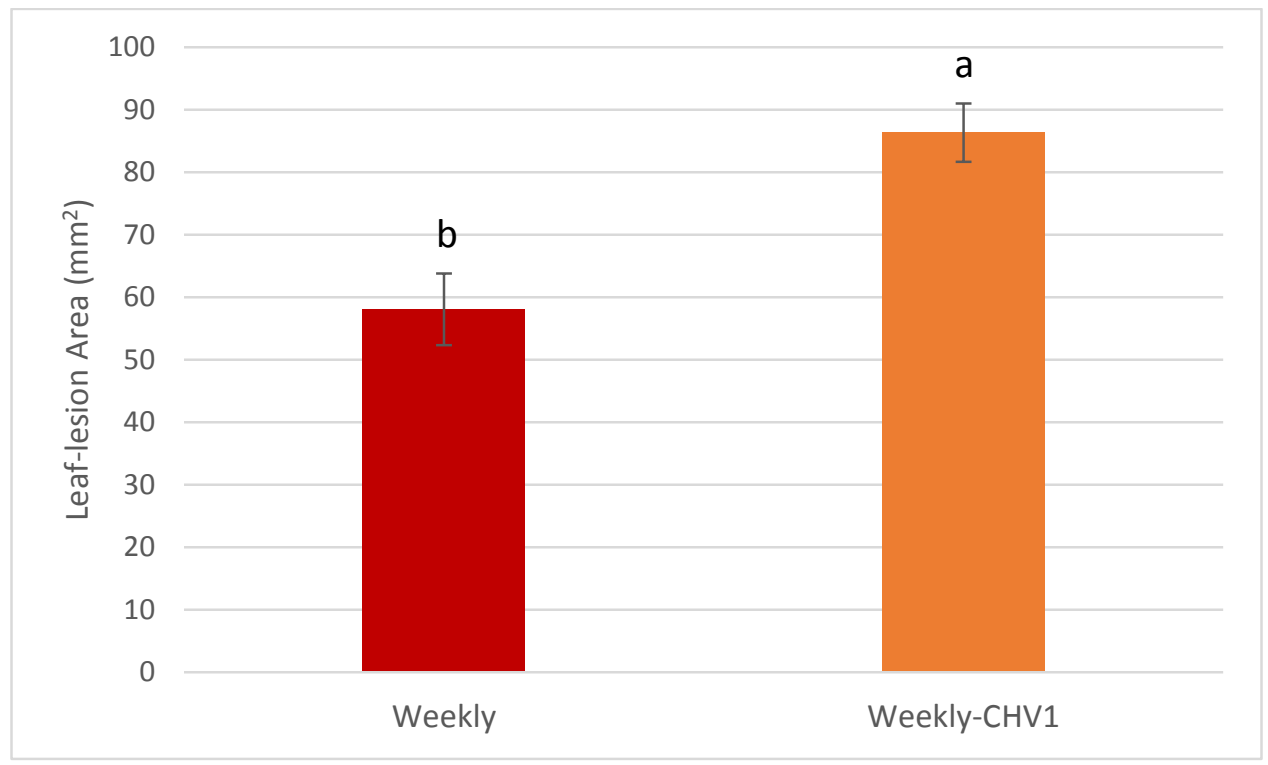

Figure 3.4: Leaf-lesion area $\left(\mathrm{mm}^{2}\right)$ averaged for all hosts for Weekly and Weekly-CHV1 inoculations; error bars represent +/- 1 SE 


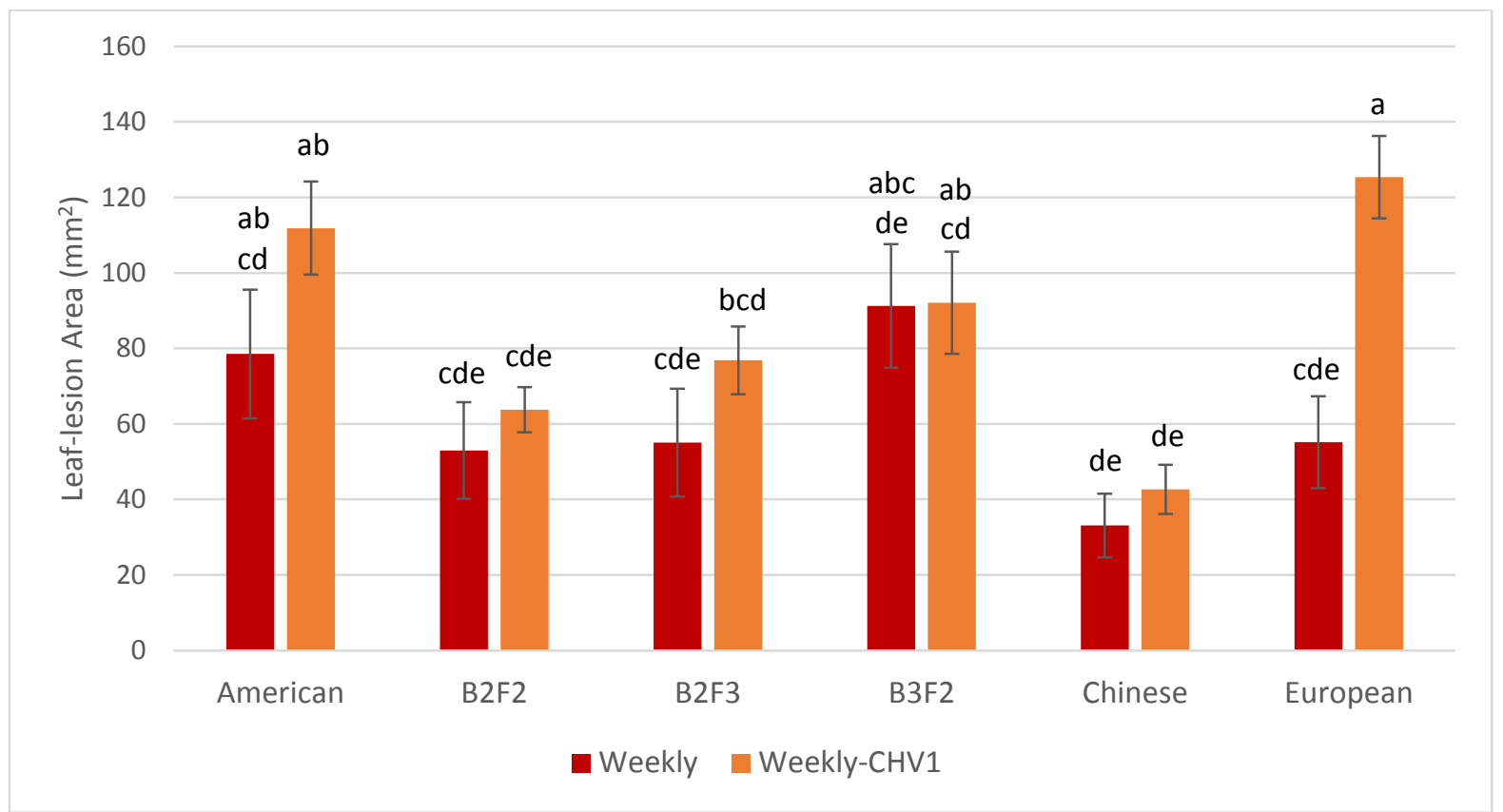

Figure 3.5: Average leaf-lesion area $\left(\mathrm{mm}^{2}\right)$ for each host backgrounds for Weekly and WeeklyCHV1 inoculations; error bars represent +/- 1 SE

\section{Lesion area by host background}

There were no significant differences in leaf-lesion area among the hosts included in the virulent Weekly inoculations assays (Figure 3.6). Chinese had a lower average lesion area than all other chestnuts with an average lesion size of $33.1 \mathrm{~mm}^{2}$. B3F2 had the largest average lesion size of $91.3 \mathrm{~mm}^{2}$. 


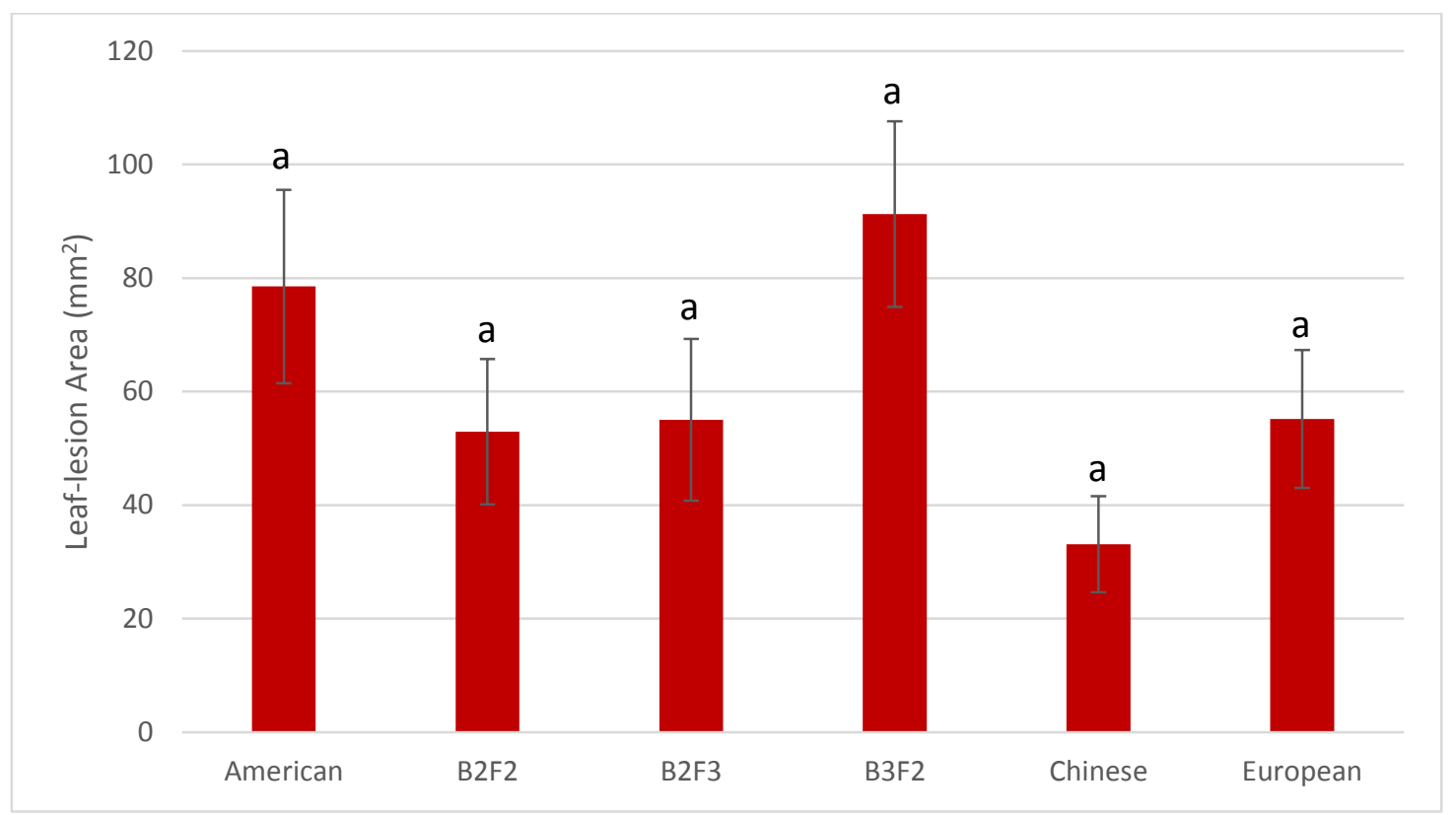

Figure 3.6: Average leaf-lesion areas $\left(\mathrm{mm}^{2}\right)$ for virulent Weekly inoculations for each host background; error bars represent +/- $1 \mathrm{SE}$

In contrast, several significant differences were identified for Weekly-CHV1 inoculations. European leaves produced a significantly larger lesion areas $\left(125.4 \mathrm{~mm}^{2}\right)$ than B2F2, B2F3, and Chinese leaves (Figure 3.7). American leaves had significantly larger lesion sizes (111.9 $\mathrm{mm}^{2}$ ) than B2F2 and Chinese. B2F2, B2F3, and B3F2 chestnut leaves resulted in similar lesion sizes of 63.7, 76.8, and $92.1 \mathrm{~mm}^{2}$, respectively. Chinese leaves had a significantly lower average lesion size than all other hosts except B2F2 and B2F3 at $42.7 \mathrm{~mm}^{2}$. 


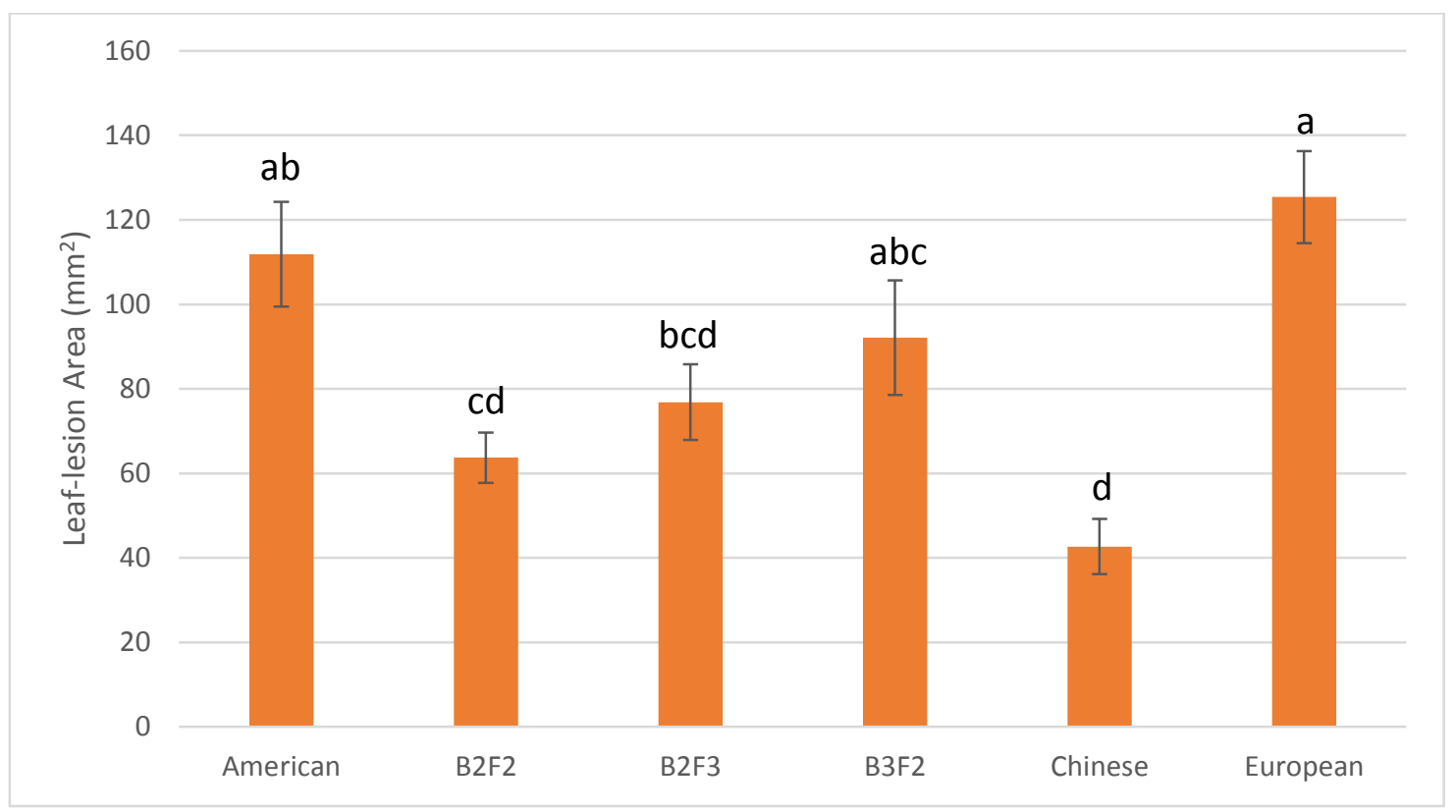

Figure 3.7: Average leaf-lesion area for Weekly-CHV1 inoculations across all host backgrounds; error bars represent +/- $1 \mathrm{SE}$

\section{Comparison of normalized canker and leaf-lesion areas}

Each tree included in this study also was screened for resistance, as described in Chapter 2, using canker measurements after artificial field inoculations of stems with Weekly and Weekly-CHV1. Those data were compared to the data resulting from this excised-leaf assay. This was achieved by dividing the linear growth or leaf-lesion area by the overall average for the American chestnuts to normalize the data. There was little correlation between normalized leaf-lesion and linear growth $(r=0.042)$. Significant differences between the normalized values for leaf-lesion and linear were found for B2F2, and all other hosts had statistically similar normalized values (Figure 3.8). Generally, a larger amount of variation existed for cankers when compared to variation of normalized leaf-lesion values. 


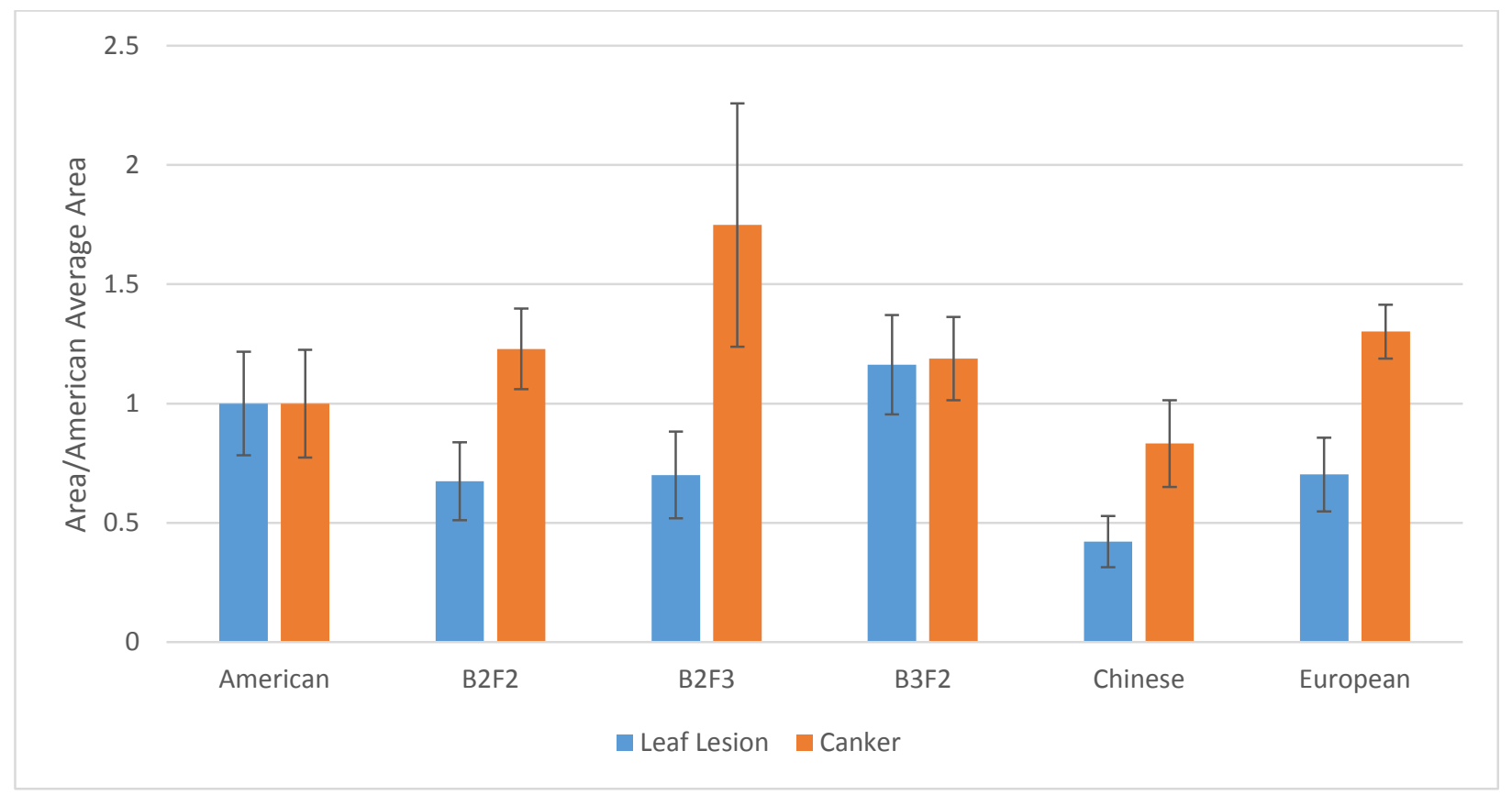

Figure 3.8: Normalized values for Weekly leaf-lesion area and Weekly linear growth measurements across all host backgrounds; error bars represent +/- $1 \mathrm{SE}$

\section{$\underline{\text { Reisolation }}$}

Fungal growth that resulted from sampled tissue was morphologically identical to the initial inoculum used for the associated treatment. All plugs transferred from failed inoculation sites resulted in the growth of the appropriate isolate. 


\section{DISCUSSION}

Resistance assays are the foundation for breeding programs such as those conducted by TACF. These assays allow for generations of trees to be screened for resistance as to eliminate individuals that do not express the desired level of resistance. Traditional programs using direct stem inoculation assays require waiting up to 5 years as the trees grow large enough to be suitable for testing. The excised-leaf assay serves as a screening method that can be completed using leaves from the first year's growth of a seedling. Therefore, less resistant individuals can be culled sooner and resources can be allocated for other uses.

In 2014, Newhouse et al. determined that the excised-leaf assay could distinguish among individuals expressing large differences in resistance (e.g. American chestnut v. Chinese chestnut). Intermediate differences in resistance also were detected using this assay (American chestnut v. Allegheny chinkapin v. Chinese chestnut). Further, the assay was able to distinguish differences in the virulence of a virulent strain (EP155) and a hypovirulent strain (EP713). Therefore, the assay was shown to be a viable screening method for blight resistance for such objectives.

In Newhouse's study, the hosts comprised approximately seven American chestnut and three Chinese chestnuts individuals grown in controlled environments, such as a growth chamber or greenhouse (Newhouse et al., 2014). The current study was designed to test the usefulness of the excised-leaf assay method on a larger number field grown trees of host backgrounds including TACF hybrid generations that should express intermediate resistance between American and Chinese chestnuts. 
The leaf-lesion areas that developed were similar to that reported by Newhouse et al. (2014). Large lesions occurred on American chestnut leaves while smaller lesions occurred on Chinese chestnut leaves when inoculated with either Weekly or Weekly-CHV1 isolates. For Weekly-CHV1, the average leaf-lesion areas on the B2F2 and B2F3 hybrid generations were significantly lower than on American but not significantly larger than on Chinese (although having a higher average). Therefore, intermediate levels of resistance were observed in the B3F2 and B2F3 generations. Based on these data, the leaf assay performed as expected even using outside leaves which have been described as not being ideal (Newhouse et al., 2014).

The percent of successful infection revealed a higher success rate for Weekly-CHV1 inoculations than compared to Weekly virulent. Failure of infection by an isolate of C. parasitica may result from the failure to make firm contact between the plug and inoculation site or inadequate moisture within the moisture chamber leading to early desiccation of the fungal plug (Newhouse et. al, 2014). In this study, failed inoculations had viable plugs on the inoculation site for the 5-day duration of the assay, which was confirmed through plating after the termination of the experiment. Further, all plugs were placed on the midvein in the same manner. Given the lack of experimental differences among treatments, the higher rate of success by Weekly-CHV1 may be the result of variation in the moisture, temperature, or another experimental factor required for growth and colonization than that required by Weekly.

Weekly-CHV1 produced significantly larger leaf-lesions than virulent Weekly. This was a surprising result given the outcome of the direct stem inoculations in which Weekly-CHV1 grew minimally and ceased growth within two-months while Weekly virulent produced significantly 
larger cankers which continued to develop over the one-year course of the study (Chapter 2). Further, this finding contradicts most if not all previous studies which show that acquiring hypovirus leads to slower, debilitated growth. To address these conflicting observations, a series of assays were used to explore the level of virulence expressed by hypovirulent isolates on chestnut and non-chestnut substrates and are reported in Chapter 4.

The larger lesions and higher rate of infection produced from Weekly-CHV1 indicate the possibility of misrepresenting results when using the excised-leaf assay for isolate virulence assays. While other isolates may produce results which more accurately represent the level of an isolate's virulence, these findings indicate the need for the addition of previously vetted isolate pairs, such as EP155 and EP713, to serve as controls when using this assay for blight resistance screening purposes (Newhouse et. al, 2014).

While these findings seem to bring the validity of the leaf assay into question, the method has been previously validated under more highly controlled conditions (Newhouse et. al, 2014). Despite efforts to select leaves such as those described by Newhouse, there were other biotic (microorganisms) and abiotic (temperature, wind, etc) factors which likely influenced the development and characteristics of the leaves leading to larger variation in leaf response to the infection. Finally, the use of vetted isolates, such as EP155 and EP713, may reduce overall variation as the method was designed using these two isolates.

The suitability of the leaf assay as a method of screening for blight resistance among chestnut hosts depends on a number of variables that determine the practicality, accuracy, and efficiency of the assay. The method was tested on a group of trees that had been previously screened for resistance using the traditional method of measuring cankers resulting from 
artificial stem inoculations. The leaf assay results were compared to canker measurements taken 12-months post-inoculation to assess how accurately the leaf assay results reflect the overall resistance expressed by the hosts one-year post inoculation.

For assays with both virulent Weekly and hypovirulent Weekly-CHV1 strains, lesion areas approximately placed each species and hybrid into their expected ranks with Chinese being most resistant and American being most susceptible (Figures 3.5 \& 3.6) (Graves, 1950; Anagnostakis, 1991; Berry, 1960; Clapper, 1951; Newhouse et al., 2014). Despite this finding, the leaf-lesion areas often were not proportional to the linear growth measurements recorded for the trees from which the leaves were collected (Figure 3.8). For B2F2, B2F3, and European, the average leaf-lesion area was smaller than American while the average linear growth measurements for those B2F2, B2F3, and European trees were larger than American. Therefore, the leaf assay results often do not reflect the results obtained from direct stem inoculations, and this brings the overall validity of the assay into question.

The leaf assay has the potential to provide results in a fraction of the time required for other blight resistance screening methods. For screening purposes, large differences in host resistance may be observed through this method, but higher replication and strict adherence to the leaf assay protocol as described by Newhouse may be necessary to elucidate intermediate levels of resistance (2014). Based on the results presented here, discriminating among similar levels of blight resistance or defining pathogen virulence may be impractical when using the leaf assay. Therefore, this assay should be reserved for rough estimations of host resistance and compared with significantly different controls such as American and Chinese. 


\section{SUMMARY}

The comparison of host resistance is a vital tool in the effort to breed for resistance to chestnut blight. Traditionally, direct-stem inoculations have been used for resistance testing, but a reduction of the time prior to screening as well as the short duration of the assay would serve as a valuable resource for breeding programs. As breeding programs often work with trees that are grown outside of controlled, greenhouse environments, the use of the leaf assay for outside-grown trees needed validation. Here, large variations in average leaf-lesion area were observed within host backgrounds as well as for individual trees. Overall, the averages were descriptive of susceptibility classes as previously described, but no correlation was found when the resultant leaf-lesions were compared to canker linear growth for each respective tree. Given these findings, the use of the leaf assay for screening efforts of outside-grown trees may be completed for rough resistance screening efforts. 


\section{LITERATURE CITED}

Anagnostakis, S. L. 1991. Measuring resistance of chestnut trees to chestnut blight. Canadian Journal of Forest Research. 22:568-71.

Berry, F. H. 1960. Relative resistance of some chestnut species and hybrids inoculated with the blight fungus. Plant Disease Reporter. 44(9):716-17.

Biraghi, A. 1953. Possible active resistance to Endothia parastitica in Castanea sativa. Reports of the International Union of Forest Research Organization. 643-45.

Elliston, J. E. 1978. Pathogenicity and sporulation in normal and diseases strains of Endothia parasitica in American chestnut. In Proceedings of the American Chestnut Symposium. W.L. MacDonald, F.C. Cech, J. Luchok, and C. Smith, eds. WV University Books, Morgantown. 110-114.

Fulbright, D. W. 1984. Effect of eliminating dsRNA in hypovirulent Endothia parasitica. Phytopathology. 74(6): 722-24.

Graves, A. H. 1950. Relative blight resistance in species and hybrids of Castanea. Phytopathology. 40(12): 1125-31.

Hebard, F. 1994. The American Chestnut Foundation breeding plan: Beginning and intermediate steps. Journal of the American Chestnut Foundation. 8:21-28.

Hebard, F. 2005. The backcross breeding program of the American Chestnut Foundation. Journal of the American Chestnut Foundation. 19(2):55-78.

Jaynes, R. A., \& Elliston, J. E. 1978. Control of Endothia parasitica cankers on American 
chestnut sprouts with hypovirulent strains. In Proceedings of the American Chestnut Symposium. W.L. MacDonald, F.C. Cech, J. Luchok, and C. Smith, eds. WV University Books, Morgantown. 110-114.

JMP®, Version 10. SAS Institute Inc., Cary, NC, 1989-2007.

Lamari, L. 2002. ASSESS: Image Analysis Software for Plant Disease Quantification. The American Phytopathological Society, St. Paul, MN.

MacDonald, W. L. and D. W. Fulbright. 1991. Biological control of chestnut blight: use and limitations of transmissible hypovirulence. Plant Disease. 75(7):656-61.

Merkel, H. W. 1905. A deadly fungus on the American chestnut. New York Zoological Society, $10^{\text {th }}$ Annual Report, pp. 97-103.

Milgroom, M. G. and P. Cortesi. 1999. Analysis of population structure of the chestnut blight fungus based on vegetative incompatibility genotypes. Proceedings of the National Academy of Science 96:10518-523.

Newhouse, A. E., J. E. Spitzer, C. A. Maynard, and W. A. Powell. 2014. Chestnut leaf inoculation assay as a rapid predictor of blight susceptibility. Plant Disease. 98(1):4-9.

Newhouse, A. E., L. D. Polin-McGuigan, K. A. Baier, K. E. R. Valletta, W. H. Rottmann, T. J. Tschaplinski, C. A. Maynard, and W. A. Powell. 2014. Transgenic American chestnuts show enhanced blight resistance and transmit the trait to T1 progeny. Plant Science. 228. 88-97.

Prospero, S., M. Conedera, U. Heiniger, and D. Rigling. 2006. Saprophytic activity and sporulation of Cryphonectria parasitica on dead chestnut wood in forests with naturally established hypovirulence. Phytopathology. 96(12):1337-44. 
Short, D. P. G., M. Double, D. L. Nuss, C. M. Stauder, W. L. MacDonald, and M. T. Kasson. 2015. Multilocus PCR Assays Elucidate Vegetative Incompatibility Gene Profiles of Cryphonectria parasitica in the United States. Applied and Environmental Microbiology. 81(17): 5736-42.

Zhang, B., A. D. Oakes, A. E. Newhouse, K. M. Baier, C. A. Maynard, and W. A. Powell. 2013. A threshold level of oxalate oxidase transgene expression reduces Cryphonectria parasitica-induced necrosis in a transgenic American chestnut (Castanea dentata) leaf bioassay. Transgenic Research. 22(5): 973-82. 


\section{CHAPTER 4: THE INVASIVENESS OF VIRULENT AND HYPOVIRULENT (EURO7-CHV1) STRAINS OF CRYPHONECTRIA PARASITICA ON HOST AND NON-HOST SUBSTRATES ABSTRACT}

Hypoviruses of Cryphonectria parasitica have been demonstrated as biological control agents against the devastating canker disease known as chestnut blight. Upon the acquisition of a hypovirus, C. parasitica often is debilitated in its growth and sporulation capability. As a result, the tree is able to resist further growth by the fungus and subsequent expansion of the canker by forming callus tissue. The degree of debilitation resulting from hypovirus infection is dependent on the interaction of the virus and the pathogen's genetics. The variation in virulence expressed by hypovirulent isolates of varying genotypes was explored through livestem inoculations. Limited variation was found among the hypovirulent strains tested, but all produced significantly smaller cankers than those caused by the included virulent isolate. In an excised-leaf and apple assay, the level of virulence expressed by virulent and hypovirulent isogenic pairs was compared. The virulent strain produced a larger lesion than its hypovirulent counterpart with one exception. One hypovirulent strain designated 'Weekly-CHV1' produced larger lesions than its isogenic, virus-free counterpart thus indicating a higher rate of growth in the absence of chestnut host resistance when carrying the hypovirus. This finding is unusual and has not been described to date. This observation may lead to further discovery of unique interactions between C. parasitica and hypoviruses. 


\section{INTRODUCTION}

Hypoviruses have been demonstrated as biological control agents of the devastating canker disease known as chestnut blight. This disease begins as an infection of a stem wound by Cryphonectria parasitica. The fungus grows radially from the point of infection killing the living tissue ahead of its growth. When C. parasitica becomes infected by a hypovirus, the chestnut blight pathogen often is debilitated to the extent that the host's response to infection is adequate to resist further growth of the fungus (MacDonald and Fulbright, 1991). The level of debilitation depends on the interactions between the virus and the pathogen, which are genetically controlled (Nuss, 2005). Therefore, the effectiveness of hypoviruses as biocontrol agents is dependent on their effect on the pathogen and the resistance expressed by the host.

To date, four species of hypovirus have been associated with $C$. parasitica with varying effects on the fitness of the fungus: Cryphonectria hypovirus 1 (CHV1) (Allemann et al., 1999; Heiniger and Rigling, 1994), CHV2 (Chung et al., 1994; Hillman, 1994), CHV3 (Smart et al., 1999), and CHV4 (Linder-Basso, 2005). While the effects of hypovirulence have been described, the variation in debilitation among individual strains has not been extensively studied. Generally, hypovirulent strains have been shown to be less virulent than their virus-free counterparts. Additionally, some level in variation of virulence has been shown among isogenic hypovirulent strains containing different viruses (Bauman, 2015).

Traditionally, chestnut host resistance and C. parasitica virulence tests have been performed on woody chestnut substrates. At least two additional substrates have been described as being useful for these experiments, which are the use of excised chestnut leaves for resistance and virulence tests and apples for pathogen virulence tests. While C. parasitica is 
not a leaf or fruit pathogen, it can colonize these tissues under certain environmental conditions while providing results similar to those from tests with woody chestnut stems thus making both substrates useful for laboratory experiments.

In two separate experiments (Chapters 2 and 3), direct inoculations in living stems and excised-leaf infections initiated with a virulent and Euro7-CHV1 containing strain of $C$. parasitica designated as 'Weekly' and 'Weekly-CHV1' produced conflicting results. In a live-stem assay conducted on plantation chestnut trees, Weekly-CHV1 grew as expected with minimal expansion followed by cessation of growth after two months while Weekly virulent continued to grow throughout the 12-month study period. Interestingly, the results of the excised-leaf assay using leaves from a selection of trees previously used in the live-stem assay indicated the virulence of Weekly without virus to be lower than that of Weekly-CHV1. Through two studies, the virulence of CHV1 containing isolates was explored to address the conflicting results described above. The first study explored the variation in virulence of genetically different isolates containing the Euro7-CHV1 hypovirus using live-stem inoculations on a clonal set of American chestnut sprouts. The second study addressed the variation in virulence among isogenic virulent and hypovirulent strains of $C$. parasitica via a leaf and an apple assay. These two objectives compared the limited growth of "Weekly-CHV1" (Chapter 2) in relation to other hypovirulent isolates while addressing its abnormal growth on chestnut leaves (Chapter 3). 


\section{MATERIALS AND METHODS}

Selection of material for live-stem assay and hypovirus transmission

Six CHV1-containing isolates were selected for the live-stem inoculations to compare the virulence expressed by geographically and genetically unique hypovirulent strains. The hypovirus designated 'Euro7' was chosen as it was previously used in the separate studies. To facilitate the transmission of this hypovirus, Weekly-CHV1 was paired with other isolates of the Weekly (EU15) vegetative incompatibility (vic) genotype from various geographic locations (Table 4.1). Matching vic genotypes aided in the transmission of hypovirus as hyphal anastomosis does not readily occur between isolates of differing vic genotypes. Vic genotypes were confirmed using a PCR method developed by Short et al. (2016). The CHV1 hypovirus, Euro7, was readily transmitted to all selected isolates via the pairing assay previously described (Chapter 2). An additional isolate named Euro7-CHV1 was included in this assay as the 'Euro7CHV1' hypovirus was originally isolated this strain. A modified dsRNA isolation protocol previously described by Morris and Dodds (1979) (Method 1) was used with previously described modifications (Chapter 2) for dsRNA confirmation (Figure 4.1).

Table 4.1: Designation and geographic origin of isolates included in the stem assay

\begin{tabular}{|c|c|}
\hline Strain Designation & Isolate Origin \\
\hline Weekly & West Virginia \\
\hline Weekly-CHV1 & West Virginia \\
\hline EU15-CHV1 & Europe \\
\hline Euro7-CHV1 & Europe \\
\hline Little Green Brier 9 (LGB9-CHV1) & Tennessee \\
\hline Sugarlands 9 (SL9-CHV1) & Tennessee \\
\hline West Salem 15 (WS15-CHV1) & Wisconsin \\
\hline
\end{tabular}




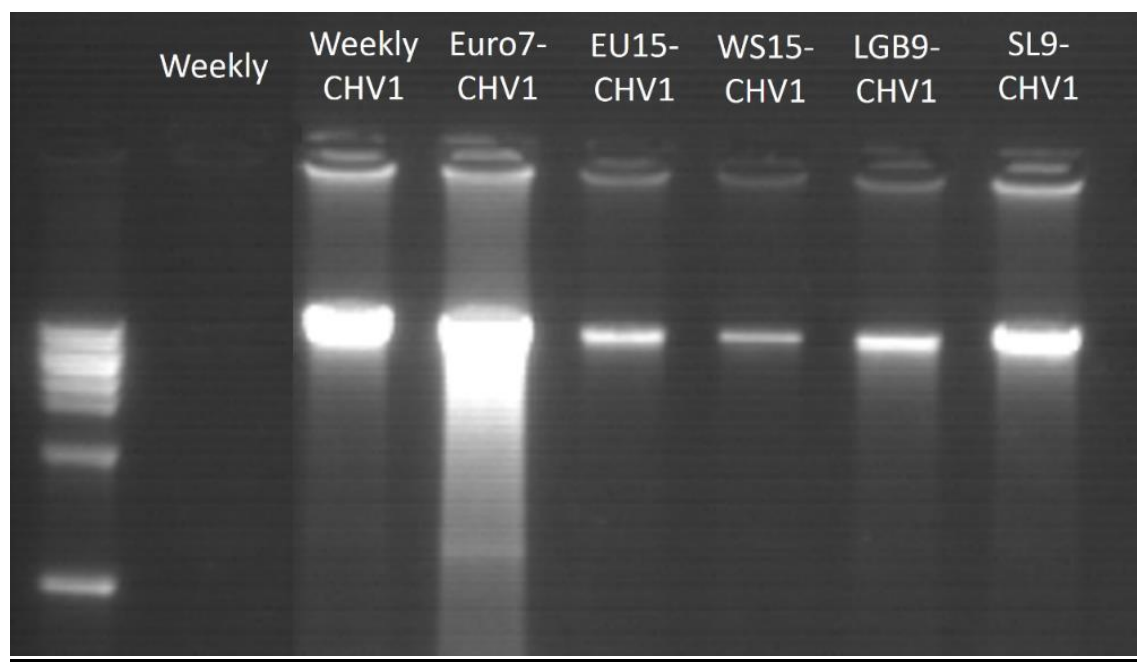

Figure 4.1: dsRNA extraction gel photo for hypovirulent isolates with virulent Weekly as a negative control

\section{Live-stem assay design}

A clump of wild American chestnut sprouts located in the Savage River State Forest in Grantsville, Maryland was selected for this assay. This clump was assumed to be clonal as all stems were growing from the same stump remnants. Five stems were selected from the clump. On each stem, a single inoculation site was made for each included inoculum for a total of seven inoculations per stem. All inoculation sites were randomized on each stem.

\section{Live-stem inoculum preparation}

All isolates were grown on Difco PDA $(39 \mathrm{~g} / 1000 \mathrm{ml})$ for approximately 5 days at $\sim 20.0 \mathrm{C}$ under a 16-to-8hr light-dark cycle. Prior to inoculation, inoculum plugs were cut around the 
growing edge of the colonies or throughout a sterile PDA plate to serve as a negative control. Live-stem inoculation plugs were cut using a 1-cm leather punch.

\section{$\underline{\text { Live-stem inoculation procedure }}$}

Inoculations proceeded as previously described by Jaynes and Elliston (1978). A 1-cm leather punch was used to create the inoculation hole to expose the vascular cambium of the stem. An inoculum plug was placed into the inoculation hole. A strip of masking tape was used to cover the inoculation hole to protect the inoculum from desiccation prior to colonization. Length and width measurements were taken using a metric ruler at approximately one month post-inoculation to inform excised-leaf and apple assay isolate selection and again, at 12-weeks post inoculation.

$\underline{\text { Excised-leaf and apple assay isolate selection and inoculum preparation }}$

In addition to the live-stem assay, excised-leaf and apple assays were used to resolve differences among isogenic virulent and hypovirulent isolate pairs. Preliminary live-stem assay results were taken one month post-inoculation and used to inform the selection of an isolate for the apple and excised-leaf assays. Although its lesions were not significantly larger than those caused by other isolates, SL9-CHV1 produced the largest average linear growth of the hypovirulent isolates included in the live-stem inoculations (Figure 4.2). Therefore, SL9-CHV1 was selected as the hypovirulent isolate to include in the leaf and apple assays as it was likely to be significantly more virulent than Weekly-CHV1. The higher level of virulence of SL9-CHV1 was desired as all other isolates had produced cankers very similar to Weekly-CHV1 and were less likely to produce significantly different results in the apple and leaf assays. 


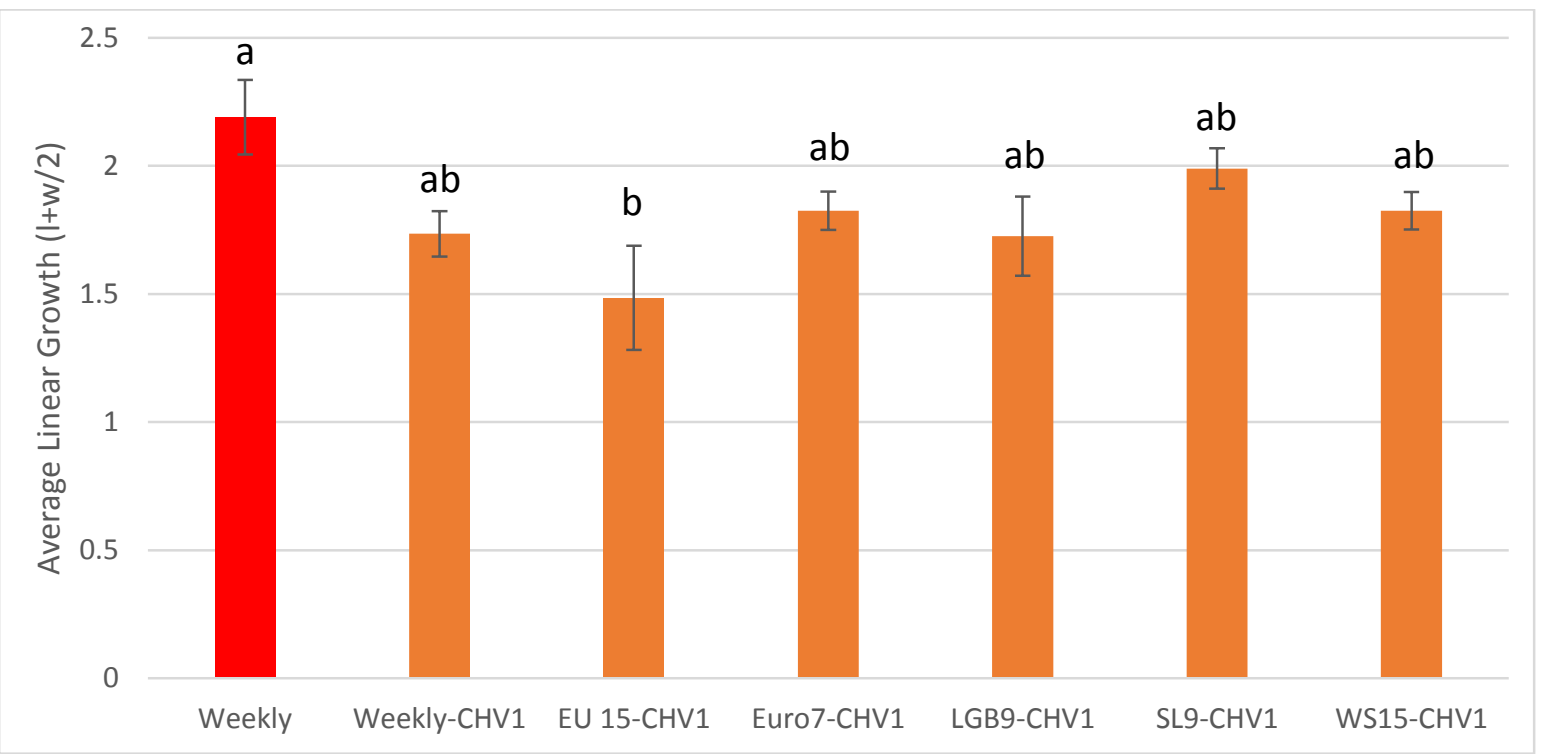

Figure 4.2: Linear growth at one month post-inoculation for virulent Weekly and six hypovirulent isolates included in the live-stem inoculations at Savage River State Forest; error bars represent +/- $1 \mathrm{SE}$

Virulent strains EP155 and hypovirulent strains EP155-CHV1 (Euro7) and EP713 (EP713) were included in addition to the virulent and hypovirulent Weekly and SL9 strain pairs as these isolates have been often selected as standard C. parasitica study isolates. EP713 was previously generated by transmitting the EP713 hypovirus into the EP155 strain. Furthermore, EP155 and EP713 were the original isolates used in the development of the excised-leaf assay as described by Newhouse (2014). The inclusion of these isolates served as references by which the leaf assay results could be compared. Isolates were grown and prepared as described above. Excised-leaf assay and apple assay plugs were cut using a \#1 cork borer, and a \#7 cork borer, respectively. 


\section{Excised-leaf assay procedure}

Forty-eight leaves were collected from the clump of American chestnut sprouts at Savage River State Forest included in the live-stem assay, placed in a cooler, and transported back to the laboratory. The leaf assay inoculation procedure was performed as previously described by Newhouse et al. (2014) and Stauder (Chapter 3). Two inoculations were included on each leaf for a total of 96 inoculations. Excised-leaf-lesion area was measured using The American Phytopathological Society: Assess 2.0 software (Lamari, 2002) six days after inoculation.

\section{Apple assay procedure}

Studies by Fulbright (1984) and Elliston (1985) previously showed success with inoculating mature, store-bought apples with C. parasitica for isolate virulence comparisons. Eleven golden delicious apples were surface disinfested in a 1:10 commercial bleach/water solution for 45 seconds then air-dried. Three inoculation sites were labeled on each apple with a Sharpie marker. A \#7 cork borer was used to create an inoculation hole at each site. A plug of the appropriate inoculum was placed into each hole and masking tape was applied to prevent inoculum desiccation prior to infection. Apples were placed inside a Tupperware bin with the lid slightly ajar to prevent excess moisture retention. After three weeks, length and width measurements were taken on the resulting lesions with a metric ruler.

Analysis

For the stem assays, linear growth was measured. Lesion area was calculated for the leaf and apple assays. All analyses were performed using JMP ${ }^{\circledR} 10$ software (SAS Institute, Inc., Cary, NC, USA). Mean comparisons served as the main analysis utilized to resolve differences 
between host backgrounds and inoculum sources. To test these factors, a one-way ANOVA with Tukey's HSD for $>3$ dependent variables $(\alpha=0.05)$ was selected to make these comparisons as only one independent variable was incorporated into each analysis.

\section{RESULTS}

\section{Live-stem assay}

Live-stem inoculations revealed few significant differences among hypovirulent isolates. Weekly virulent $(8.42 \mathrm{~cm})$ produced a significantly more average linear growth than the hypovirulent isolates. Weekly-CHV1 $(2.73 \mathrm{~cm}), \mathrm{SL9}-\mathrm{CHV} 1(4.16 \mathrm{~cm})$, and Euro7-CHV1 $(3.26 \mathrm{~cm})$ produced similar amounts of linear growth. EU15-CHV1 $(4.24 \mathrm{~cm})$ and WS15-CHV1 $(4.02 \mathrm{~cm})$ produced the largest cankers of the hypovirulent isolates tested, but these were not significantly larger compared to the other hypovirulent isolates.

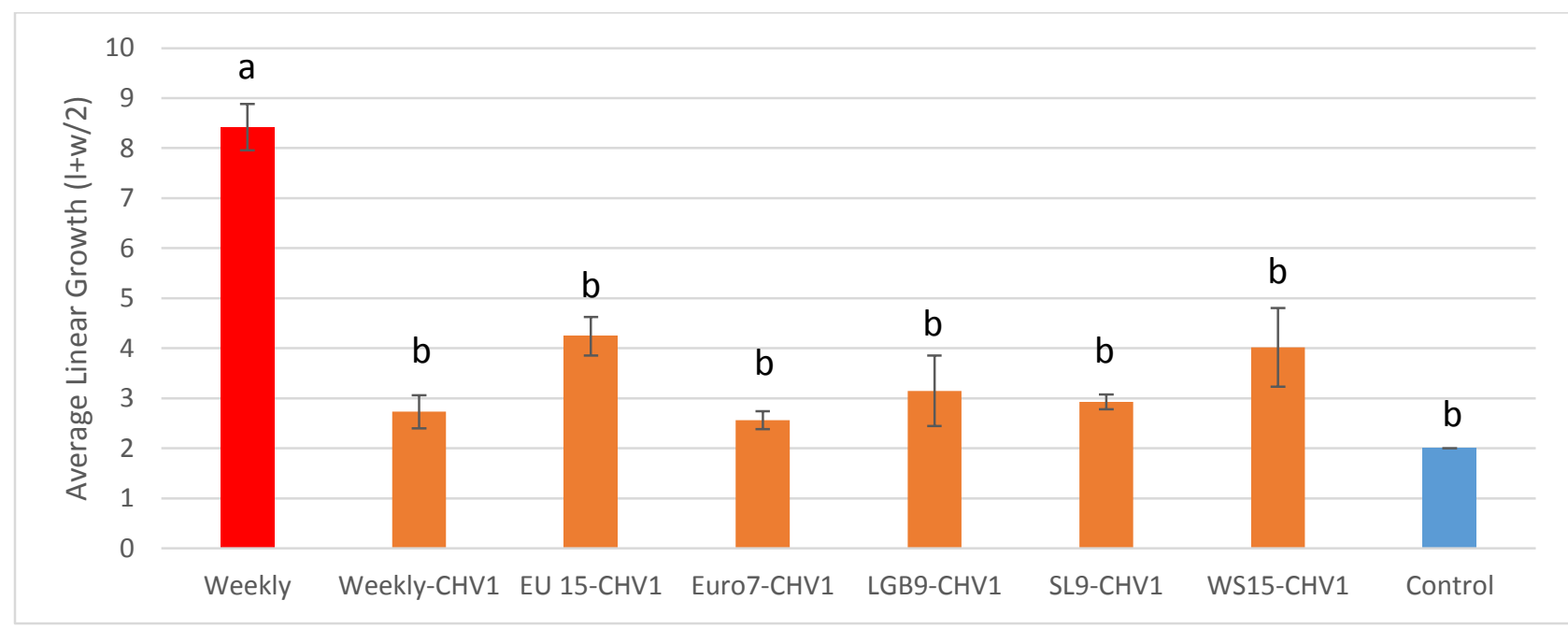

Figure 4.3: Linear growth at twelve weeks post-inoculation for virulent Weekly and six hypovirulent isolates included in the live-stem inoculations at Savage River State Forest; error bars represent +/- 1 SE 


\section{Excised-leaf assay}

The excised-leaf assay compared the relative virulence of hypovirulent isolates to their virulent counterparts as well as among virulent and hypovirulent isolate pairs. As in Chapter 3, Weekly-CHV1 produced larger lesions $\left(78.15 \mathrm{~mm}^{2}\right)$ on average, but lesion area was not significantly different than those of Weekly virulent $\left(43.76 \mathrm{~mm}^{2}\right)$. No other virulenthypovirulent pair had this trend. SL9 virulent $\left(180.72 \mathrm{~mm}^{2}\right)$ produced significantly larger lesions than SL9-CHV1 $\left(20.11 \mathrm{~mm}^{2}\right)$, and EP155 $\left(159.64 \mathrm{~mm}^{2}\right)$ produced larger lesions than EP155-CHV1 (79.47 mm²). Weekly-CHV1, EP155-CHV1, and EP713-CHV1 (76.62 $\left.\mathrm{mm}^{2}\right)$ produced larger lesions than SL9-CHV1, but differences among these values were not statistically significant.

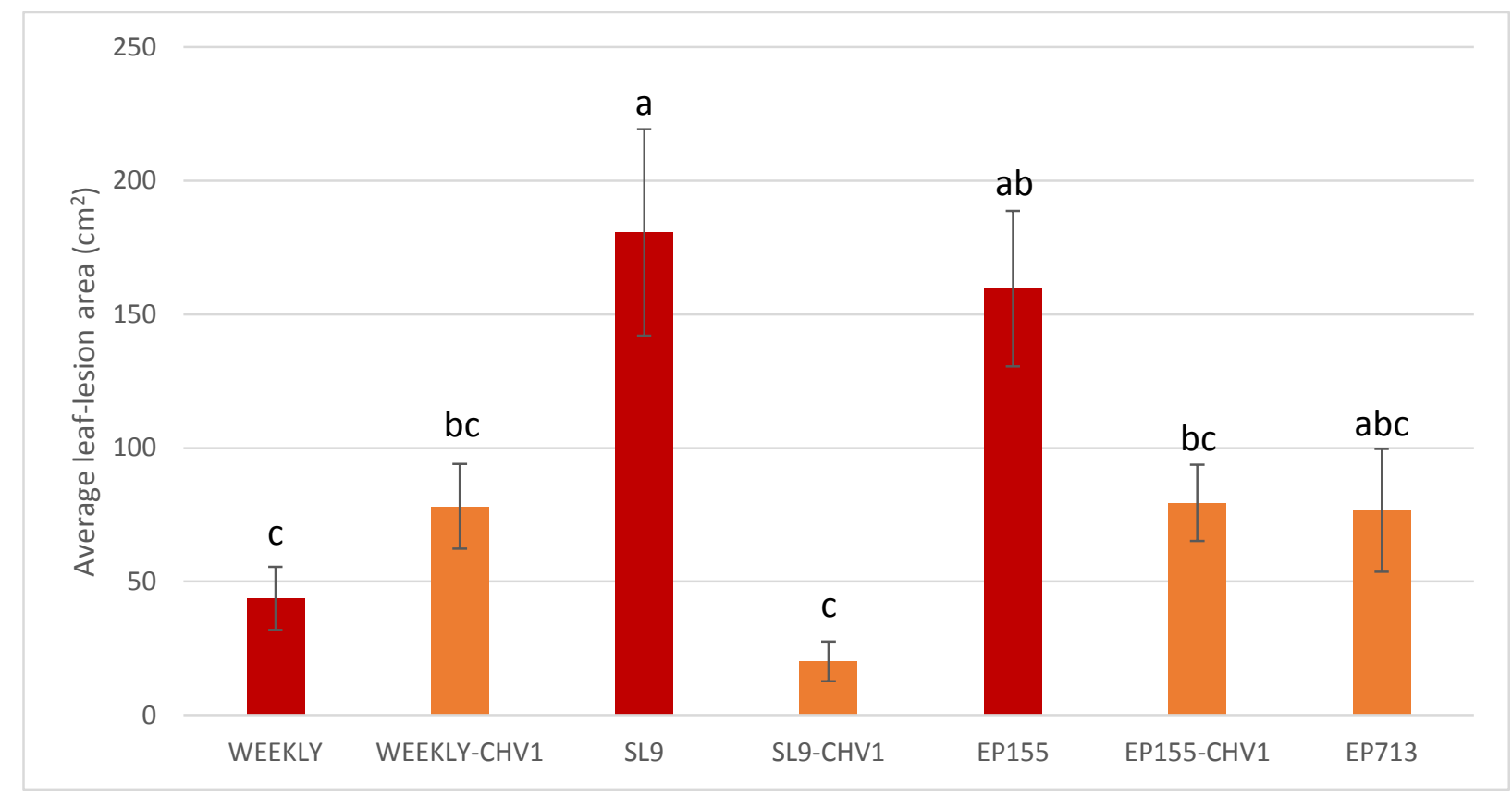

Figure 4.4: Lesion areas for isolates included in the excised-leaf assay; error bars represent +/- $1 \mathrm{SE}$ 


\section{Apple assay}

The apple assay served as a second virulence test to compare differences in lesion area between isogenic virulent and hypovirulent pairs as well as among all included isolates. Similar to the results of the excised-leaf assay, Weekly-CHV1 produced more linear growth $(4.11 \mathrm{~cm})$ than Weekly $(3.46 \mathrm{~cm})$, but these averages were not significantly different. Weekly-CHV1 produced the most average linear growth of all included isolates. For all other virulenthypovirulent pairs, the virulent isolate produced more average linear growth than its hypovirulent counterpart, but no significant differences were found between isolate pairs. All isolates produced significantly more linear growth than the control.

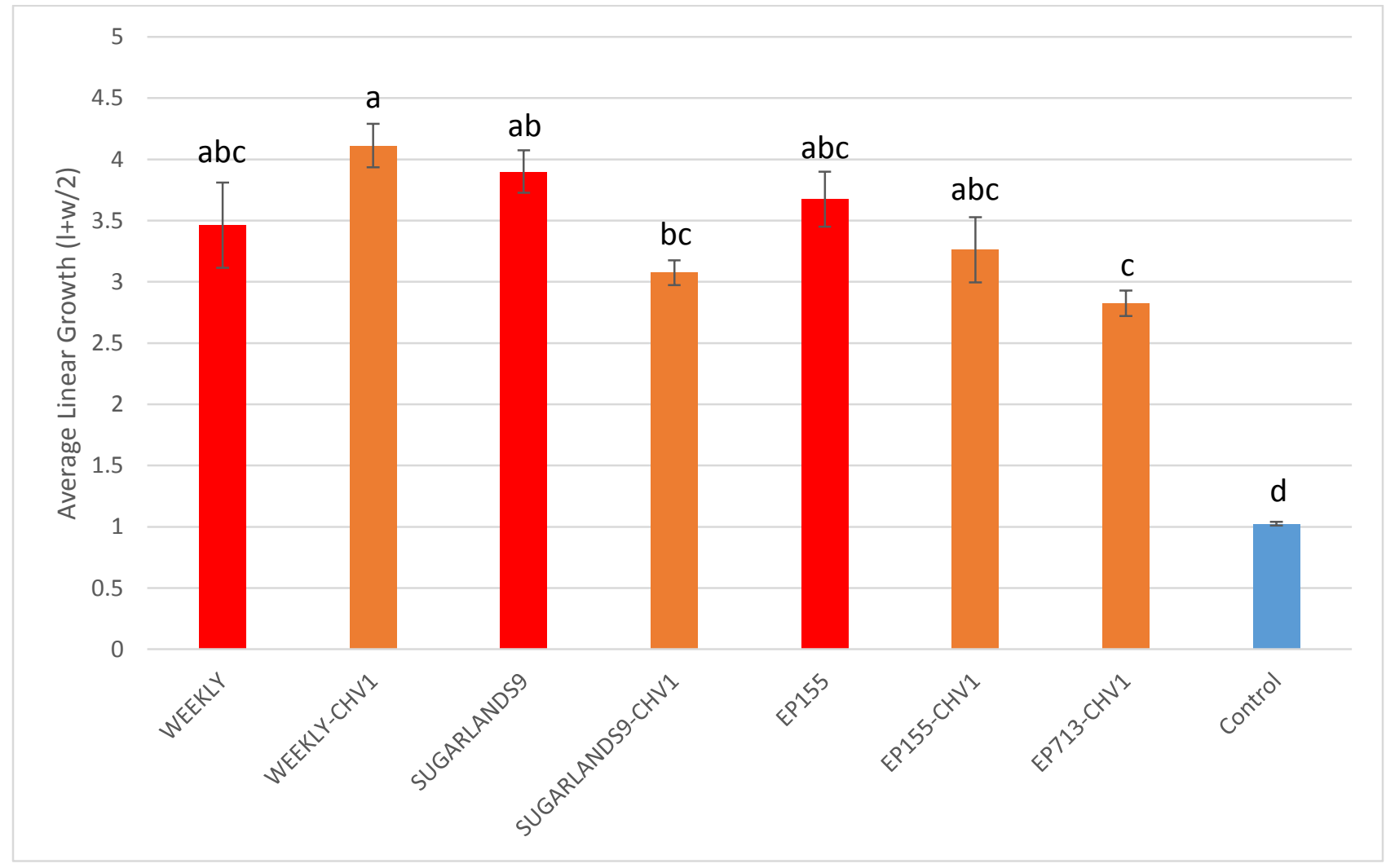

Figure 4.5: Average linear growth for all isolates included in the apple assay; Error bars represent +/- 1 SE 


\section{DISCUSSION}

The effectiveness of hypoviruses as biocontrol agents is partially dependent on the level of debilitation resulting from virus infection. In most cases, hypovirulent infections can be compartmentalized by the chestnut host through the production of callus tissue even on susceptible hosts. However, a balance between the pathogen's rate of sporulation and invasiveness is necessary for the hypovirulent isolates to disseminate throughout a landscape while not killing the host tree. For effective biocontrol, the interaction between these hypovirulent isolates and the level of resistance expressed by the host must be balanced to ensure host survival while allowing for hypovirulent isolate dissemination.

The level of virulence expressed by a selection of hypovirulent isolates was assessed through inoculations on a clump of American chestnut sprouts in the Savage River State Forest. The findings indicated that all isolates were equally debilitated when compared to virulent Weekly. EU15-CHV1 and WS15-CHV1 produced larger cankers suggesting a slightly higher level of virulence. The test captured some variation in the virulence of the hypovirulent isolates. Some differences in EU15-CHV1 and WS15-CHV1 strain-virus genetic interactions are likely responsible for this higher level of virulence given that the hypovirus in each strain was identical.

Another purpose of the live-stem inoculations was to identify a hypovirulent isolate that likely would be significantly more virulent than Weekly-CHV1. SL9-CHV1 was selected for the apple and leaf assays based on its larger average lesion size than other hypovirulent isolates at one month. Unfortunately, the subsequent growth of SL9-CHV1 was minimal compared with all other isolates and failed to be significantly more virulent than Weekly-CHV1 in this test. The 
short longevity of the initial virulence of SL9-CHV1 is suggestive of some variation in the rate of growth over time in the presence of host resistance.

The apple and leaf assays were used to compare the growth of isogenic virulent and hypovirulent strains. Weekly and Weekly-CHV1 isolates produced results similar to those described in Chapter 3. The hypovirulent Weekly-CHV1 produced larger lesions than Weekly virulent in both assays. Although these values were not significantly different, this finding raises numerous questions regarding the interaction of the Euro7 hypovirus and Weekly isolate. The greater growth of Weekly-CHV1 on non-woody chestnut substrates seems to be unique as the live-stem inoculations were similar to those results described in Chapter 2 in which WeeklyCHV1 ceased growth while Weekly virulent continued to grow throughout the study period.

EP155 and EP713 were included in the excised-leaf and apple assays to serve as controls as their virulence has been previously described (Newhouse et al., 2014). In the Newhouse et al. study (2014), EP155 produced significantly larger leaf-lesions than EP713. Similar trends were observed for both the excised-leaf and apple assays included here. Therefore, the observations for Weekly and Weekly-CHV1 likely are not a result of the assay, but instead, an interaction with Weekly and the Euro7 hypovirus.

Given C. parasitica's ability to grow on non-woody, non-chestnut substrates, the role of growth on non-host substrates in hypovirus dissemination should be considered as valuable in the use of hypoviruses as biocontrols of chestnut blight. Here, Weekly-CHV1 had greater growth under these experimental conditions than Weekly virulent. Based on these findings, it could be assumed that Weekly-CHV1 has greater hypovirus dissemination potential if nonwoody, non-chestnut growth does have a role in hypovirulence as a natural biocontrol of 
chestnut blight. Therefore, the identification of other hypovirulent isolates which grow at such rates may be useful in the selection of isolates used for biocontrol as they may help promote hypovirus dissemination.

Although there was no observation or measurement of sporulation in the apple and leaf assays, $C$. parasitica is capable of growth on non-host substrates. Additionally, its saprophytic growth capabilities have been previously documented. Goddard et. al (2015) reported C. parasitica as a weak saprophyte with higher rates of growth and sporulation for virulent isolates than hypovirulent isolates. This finding is not surprising as hypovirulent isolates are commonly associated with lower rates of growth and sporulation. Here, a hypovirulent isolate had greater growth potential on non-host substrates. While Weekly-CHV1 may be unique in these characteristics, the presence of additional hypovirulent isolates which exhibit this growth is equally possible. Therefore, additional studies are needed to identify additional hypovirulent isolates which exhibit this higher rate of non-host growth and explore their saprophytic growth and sporulation capabilities. 


\section{SUMMARY}

The virulence of hypovirulent isolates has many implications on the success of hypovirulence as a biocontrol. In this study, limited variation was observed among the hypovirulent isolates included in the live-stem inoculations. This finding indicates that the potential for substantial growth and sporulation by hypovirulent isolates is severely reduced due to the debilitation by the hypovirus regardless of genotype. Despite the limited variation observed within the chestnut stems, the increased growth of Weekly-CHV1 observed in the excised-leaf and apple assays appears to be indicative of a unique interaction between the $C$. parasitica and the hypovirus. No other hypovirulent isolate included in this study produced larger lesions than its virulent counterpart, but this increased growth may not be unique to Weekly-CHV1. An increased rate of growth on non-woody, non-chestnut substrates is likely indicative of a higher rate of sporulation and therefore, hypovirus dissemination. Due to this, the growth potential of hypovirulent isolates should be considered for the use of hypovirulence as a biocontrol. 


\section{LITERATURE CITED}

Allemann, C., P. Hoegger, U. Heiniger, and D. Rigling. 1999. Genetic variation of Cryphonectria hypoviruses (CHV1) in Europe, assessed using restriction fragment length polymorphisms (RFLP) markers. Molecular Ecololgy. 8(5):843-54.

Bauman, J. M. 2015. A comparison of the growth and asexual reproduction by Cryphonectria parastitica isolates infected with hypoviruses CHV3-County Line, CHV1Euro7, and CHV1-Ep713. American Journal of Plant Sciences. 6:73-83.

Chung, P., P. J. Bedker, and B. I. Hillman. 1994. Diversity of Cryphonectria parasitica hypovirulence-associated double-stranded RNAs within a chestnut population in New Jersey. Phytopathology. 84(9): 984-990.

Elliston, J. E. 1985. Characteristics of dsRNA-free and dsRNA-containing strains of Endothia parasitica in relation to hypovirulence. Phytopathology. 75:151-58

Fulbright, D. W. 1984. Effect of eliminating dsRNA in hypovirulent Endothia parasitica. Phytopathology. 74(6): 722-24.

Goddard, E. S. 2015. Saprophytic Colonization and Sporulation of Virulent and Hypovirulent Cryphonectria parasitica on American Chestnut (Castanea dentata) and Scarlet Oak (Quercus coccinea). Master thesis. Davis College of Agriculture, Natural Resources, and Design. West Virginia University.

Heiniger, U. and D. Rigling. 1994. Biological control of chestnut blight in Europe. Annual Review of Phytopathology. 32:581-99.

Hillman, B. I., B. T. Halpern, and M. P. Brown. 1994. A viral dsRNA element of the chestnut blight fungus with a distinct genetic organization. Virology. 201(2):241-50. 
Jaynes, R. A., \& Elliston, J. E. 1978. Control of Endothia parasitica cankers on American chestnut sprouts with hypovirulent strains. In Proceedings of the American Chestnut Symposium. W.L. MacDonald, F.C. Cech, J. Luchok, and C. Smith, eds. WV Univ. Books, Morgantown. 110-114.

JMP®, Version 10. SAS Institute Inc., Cary, NC, 1989-2007.

Lamari, L. 2002. ASSESS: Image Analysis Software for Plant Disease Quantification. The American Phytopathological Society, St. Paul, MN.

Linder-Basso, D., J. N. Dynek, and B. I. Hillman. 2005. Genome analysis of Cryphonectria hypovirus 4, the most common hypovirus species in North America. Virology. 1:193203.

MacDonald, W. L. and D. W. Fulbright. 1991. Biological control of chestnut blight: use and limitations of transmissible hypovirulence. Plant Disease. 75(7):656-61.

Morris, J. T. and J. A. Dodds. 1979. Isolation and analysis of double-stranded RNA from virusinfected plant and fungal tissue. Phytopathology. 69(8):854-58.

Newhouse, A. E., J. E. Spitzer, C. A. Maynard, and W. A. Powell. 2014. Chestnut leaf inoculation assay as a rapid predictor of blight susceptibility. Plant Disease. 98(1):4-9.

Nuss, D. L. 2005. Hypovirulence: Mycoviruses at the fungal-plant interface. Nature 3:63242.

Short, D. P. G., M. Double, D. L. Nuss, C. M. Stauder, W. L. MacDonald, and M. T. Kasson. 2015. Multilocus PCR Assays Elucidate Vegetative Incompatibility Gene Profiles of Cryphonectria parasitica in the United States. Applied and Environmental Microbiology. 81(17): 5736-42. 
Smart, C. D., W. Yuan, R. Foglia, D. L. Nuss, D. W. Fulbright, and B. I. Hillman. 1999.

Cryphonectria hypovirus 3, a virus species in the family Hypoviridae with a single open reading frame. Virology. 265(1):66-73. 


\section{THESIS SUMMARY}

The accidental introduction of Cryphonectria parasitica and its subsequent devastation of American chestnut populations in North America resulted in research efforts to control the disease of chestnut blight and restore the American chestnut to its former prominence. The use of backcross breeding by The American Chestnut Foundation (TACF) and more recently, transgenic techniques have been used in efforts to generate a blight-resistant American chestnut or closely related hybrids (Hebard, 2005; Zhang et al., 2013). In the first experiment of this study, a selection of trees including American, Chinese, European, and three generations of TACF hybrid (B2F2, B2F3, and B3F2) chestnuts were screened for resistance by direct stem inoculations on living trees with isogenic virulent and hypovirulent strains designated Weekly and Weekly-CHV1 (Euro7), respectively. All hosts aside from Chinese chestnuts were similarly susceptible to the virulent Weekly isolate as measured by linear growth, stromata count, and rate of growth measurements. Despite this finding, several lineages from within a species or hybrid generation were more resistant than others comprising that group. Subsequent studies to further explore and validate the levels of resistance observed among these lineages are needed.

In a second experiment, an excised-leaf assay method produced results that corresponded with the relative resistance of hosts used in this study despite a low incidence of infection for both Weekly and Weekly-CHV1. The most contrasting difference was with American chestnut leaves which had significantly larger lesions than Chinese chestnut leaves for both isolates. This test validated the leaf assay technique for the identification of large 
differences in resistance. Therefore this method can serve as a resistant screening method to identify individuals that are significantly similar to a Chinese chestnut control.

The virulence of $C$. parasitica also is related to its success as a pathogen. Debilitating hypoviruses can serve as biological control agents through the phenomenon termed hypovirulence. In this study, the virulence of hypovirulent isolates was severely debilitated resulting in the extreme reduction of the pathogen's invasiveness and sporulation capabilities compared to their virulent counterparts. Interestingly, Weekly-CHV1 had greater invasiveness than Weekly when infecting chestnut leaf and apple tissues. This is the first report of such an observation and raises questions regarding the hypovirus's influence on the biology of the Weekly isolate. The existence of additional hypovirulent isolates which express a higher level of invasiveness than their virulent counterparts when infecting tissue other than woody, chestnut tissue is not known.

Subsequent studies are needed to explore the variation of resistance within the lineages comprising the species and hybrid generations included in this study and to identify hypovirulent isolates that exhibit increased invasiveness on alternative substrates. As previously mentioned, the excised-leaf assay has the capability of identifying large differences in susceptibility. Therefore, the excised-leaf assay may be useful to identify the more resistant lineages planted at the WVU agronomy farm. In addition to this, the invasiveness of additional hypovirulent isolates on non-host substrates in relation to their virulent counterparts may be explored through the excised-leaf assay. The identification of additional isolates which produce results similar to Weekly and Weekly-CHV1 may provide insight into unique interactions between $C$. parasitica and its associated hypoviruses. 
Appendix A: Media Used

Potato Dextrose Agar (PDA):

- Difco $^{\circledR}$ Potato Dextrose Agar

$39.0 \mathrm{~g}$

- Distilled water

$1000 \mathrm{ml}$

Glucose Yeast Extract Agar with Antibiotics (GYE/A):

- Dextrose (glucose)

$10.0 \mathrm{~g}$

- Yeast Extract

$2.0 \mathrm{~g}$

- $\mathrm{KH}_{2} \mathrm{PO}_{4}$

$1.0 \mathrm{~g}$

- $\mathrm{MgSO}_{4}$

$0.5 \mathrm{~g}$

- Thiamine

$50 \mu \mathrm{g}$

- Biotin

$10 \mu \mathrm{g}$

- Microelements
$\bigcirc \mathrm{Fe}+++$
$500 \mu \mathrm{g}$
○ Mn++
$439 \mu \mathrm{g}$
○ $\mathrm{Zn++}$
$154 \mu \mathrm{g}$

- Agar

$20.0 \mathrm{~g}$

- Distilled water

$1000 \mathrm{ml}$

- Antibiotics (added to cooled media $\left(\sim 50^{\circ} \mathrm{C}\right)$ )
○ Tetracycline hydrochloride $100 \mathrm{mg}$
- Streptomycin sulfate
$10 \mathrm{mg}$ 